\title{
Gauge theory loop operators and Liouville theory
}

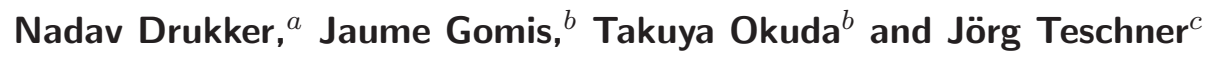 \\ ${ }^{a}$ Institut für Physik, Humboldt-Universität zu Berlin, \\ Newtonstraße 15, D-12489 Berlin, Germany \\ ${ }^{b}$ Perimeter Institute for Theoretical Physics, \\ Waterloo, Ontario, N2L 2Y5, Canada \\ ${ }^{c}$ DESY Theory, \\ Notkestraße 85, 22603 Hamburg, Germany \\ E-mail: drukker@physik.hu-berlin.de, jgomis@perimeterinstitute.ca, \\ takuya@perimeterinstitute.ca, teschner@mail.desy.de
}

Abstract: We propose a correspondence between loop operators in a family of four dimensional $\mathcal{N}=2$ gauge theories on $S^{4}$ — including Wilson, 't Hooft and dyonic operators - and Liouville theory loop operators on a Riemann surface. This extends the beautiful relation between the partition function of these $\mathcal{N}=2$ gauge theories and Liouville correlators found by Alday, Gaiotto and Tachikawa. We show that the computation of these Liouville correlators with the insertion of a Liouville loop operator reproduces Pestun's formula capturing the expectation value of a Wilson loop operator in the corresponding gauge theory. We prove that our definition of Liouville loop operators is invariant under modular transformations, which given our correspondence, implies the conjectured action of S-duality on the gauge theory loop operators. Our computations in Liouville theory make an explicit prediction for the exact expectation value of 't Hooft and dyonic loop operators in these $\mathcal{N}=2$ gauge theories. The Liouville loop operators are also found to admit a simple geometric interpretation within quantum Teichmüller theory as the quantum operators representing the length of geodesics. We study the algebra of Liouville loop operators and show that it gives evidence for our proposal as well as providing definite predictions for the operator product expansion of loop operators in gauge theory.

KEYwords: Supersymmetric gauge theory, Field Theories in Lower Dimensions, Duality in Gauge Field Theories

ArXIV ePrint: 0909.1105 


\section{Contents}

1 Introduction 1

2 Gauge theories, Liouville theory and curves on Riemann surfaces 5

2.1 Gauge theories and Liouville theory 5

2.2 Loop operators in gauge theory and curves on Riemann surfaces 9

3 Holonomy and monodromy in Liouville theory 11

4 Loop operators in gauge theories from Liouville theory $\quad \mathbf{1 5}$

$\begin{array}{lll}4.1 & \text { Loop operators in Liouville theory } & 15\end{array}$

$\begin{array}{ll}4.2 \text { Calculational scheme } & 16\end{array}$

$\begin{array}{lll}4.3 & \text { Relation to the gauge theory } & 18\end{array}$

5 Computation of gauge theory loop operators $\quad 19$

$\begin{array}{lll}5.1 \text { Preliminaries } & 19\end{array}$

5.2 Wilson loops from Liouville theory 22

5.3 't Hooft loop in $\mathcal{N}=2^{*}$ theory 25

5.4 't Hooft loop in the theory with one $\mathrm{SU}(2)_{C}$ and $N_{F}=4 \quad 27$

$\begin{array}{lll}5.5 & \text { More general loop operators } & 29\end{array}$

$\begin{array}{lll}\text { 5.5.1 Building loops from smaller constituents } & 30\end{array}$

6 The Liouville-Teichmüller theory $\quad \mathbf{3 4}$

6.1 Classical Liouville-Teichmüller theory on Riemann surfaces 34

6.1.1 Complex-analytic picture 34

$\begin{array}{lll}6.1 .2 & \text { Hyperbolic picture } & 35\end{array}$

6.1.3 Symplectic structure in the hyperbolic picture 36

$\begin{array}{ll}\text { 6.1.4 Length functions } & 36\end{array}$

$\begin{array}{ll}6.2 \text { Kähler quantization } & 37\end{array}$

$\begin{array}{ll}\text { 6.3 Quantization in a real polarization } & 38\end{array}$

$\begin{array}{ll}\text { 6.3.1 Algebra of observables and Hilbert space } & 38\end{array}$

$\begin{array}{ll}6.4 \text { Geodesic length operators } & 39\end{array}$

6.4.1 The length representation $\quad 40$

6.5 Relation between length representation and Kähler quantization 40

7 Algebra of loop operators in Liouville-Teichmüller theory 42

$\begin{array}{ll}7.1 & \text { Quantum skein relation }\end{array}$

$\begin{array}{ll}7.2 & \text { Comparison with gauge theory }\end{array} 4$

7.2.1 The commutation relations 44

$\begin{array}{lll}7.2 .2 & \text { The operator product expansion } & 46\end{array}$

$\begin{array}{lll}7.3 & \text { Examples } & 48\end{array}$

$\begin{array}{lll}\text { 7.3.1 } \mathcal{N}=2^{*} \text { theory } & 48\end{array}$ 
A.1 The function $\Gamma_{b}(x) \quad 51$

A.2 The function $S_{b}(x) \quad 51$

A.3 $\Upsilon$ function $\quad 52$

B Fusion matrices $\quad \mathbf{5 2}$

B.1 Higher degenerate fields $\quad 53$

\section{Introduction}

The concept of duality has been a major driving force in the study of lattice models, field theories as well as string theory. It is, in fact, the main idea uniting different field theories and string/M-theory. As an early example, the dual resonance model, where the search for an amplitude which can be interpreted as a sum over poles in the $s^{-}, t$ or $u$-channels, culminated in the celebrated Veneziano formula. The underlying structure, worldsheet duality, is a consequence of the modular bootstrap of two dimensional conformal field theory, which associates to an amplitude multiple inequivalent representations, each corresponding to a different sewing of the Riemann surface from pairs of pants.

Ever since, duality has proliferated in supersymmetric gauge theories and string/Mtheory as a conjectured equivalence between dual theories evaluated at different spacetime couplings. These dualities have greatly advanced our nonperturbative understanding of gauge theories and string/M-theory. An elegant example is the S-duality of four dimensional $\mathcal{N}=4$ super Yang-Mills [1-3]. The theory with gauge group $G$ conjecturally admits the action of a duality group $\Gamma_{G} \subset \mathrm{SL}(2, \mathbb{R})$, which contains elements that exchange the theory with gauge group $G$ with the theory with dual gauge group ${ }^{L} G$ [4].

Dualities are also expected to act on four dimensional $\mathcal{N}=2$ supersymmetric conformal field theories. Unlike gauge theories with $\mathcal{N}=4$ supersymmetry, which are unique given a choice of gauge group $G$, the space of four dimensional $\mathcal{N}=2$ supersymmetric conformal field theories is very rich. The first duality proposal was made for $\mathcal{N}=2 \mathrm{SU}(2)$ super Yang-Mills with 4 fundamental hypermultiplets, conjectured by Seiberg and Witten to admit the action of an $\mathrm{SL}(2, \mathbb{Z})$ duality group [5]. Other proposals for various $\mathcal{N}=2$ gauge theories have been made since then (see e.g. $[6,7]$ ), strengthening the hypothesis of duality for $\mathcal{N}=2$ supersymmetric conformal field theories.

Gaiotto [8] has recently constructed a class of $\mathcal{N}=2$ theories, denoted by $\mathcal{T}_{g, n}$, from the data associated with a genus $g$ Riemann surface with $n$-punctures, denoted by $C_{g, n} .{ }^{1}$ For

\footnotetext{
${ }^{1}$ Important ingredients for this construction are the results presented in $[7,9]$.
} 
this class of $\mathcal{N}=2$ gauge theories, which are based on $\mathrm{SU}(2)$ gauge groups, ${ }^{2}$ Gaiotto [8] has conjectured that the spacetime duality of the gauge theory $\mathcal{T}_{g, n}$ corresponds to worldsheet duality on the associated Riemann surface $C_{g, n}$ :

$$
\text { S-duality } \longleftrightarrow \text { worldsheet duality . }
$$

The construction identifies the parameter space of $\mathcal{T}_{g, n}$ with the moduli space of complex structures of $C_{g, n}$, as well as the duality group $\Gamma\left(\mathcal{T}_{g, n}\right)$ of the four dimensional gauge theory $\mathcal{T}_{g, n}$ with the mapping class group of $C_{g, n}$

$$
\Gamma\left(\mathcal{T}_{g, n}\right) \simeq \operatorname{MCG}\left(C_{g, n}\right) .
$$

Given a theory $\mathcal{T}_{g, n}$ encoded by a Riemann surface $C_{g, n}$, Gaiotto associates a generalized quiver gauge theory to a pants decomposition of $C_{g, n}$, describing the sewing of the Riemann surface from pairs of pants. A choice of pants decomposition corresponds to a choice of duality frame for $\mathcal{T}_{g, n}$. At certain corners of the moduli space of $C_{g, n}$ the surface degenerates to a collection of $3 g-3+n$ thin tubes connected to each other by pairs of pants. In such singular limits the surface reduces to a graph with tri-valent vertices, where the theory $\mathcal{T}_{g, n}$ has a weakly coupled Lagrangian description as a generalized quiver gauge theory. ${ }^{3}$ Hence, the $\mathcal{T}_{g, n}$ theory admits multiple descriptions in terms of generalized quivers, each valid in different corners of parameter space and involving different degrees of freedom.

The partition function of $\mathcal{N}=2$ gauge theories on $S^{4}$ was derived by Pestun in [10] using localization with respect to the $O s p(2 \mid 2)$ supergroup. He showed that the partition function reduces to a matrix integral over the zero modes of the scalar fields in the $\mathcal{N}=2$ vector multiplets of the product of the Nekrasov partition function $Z_{\text {Nekrasov }}[11,12]$ with its complex conjugate. The partition function has an elegant interpretation in terms of the product of a holomorphic contribution corresponding to the North pole and an antiholomorphic contribution corresponding to the South pole [10], akin to the holomorphically factorized representation of two dimensional conformal field theory correlators.

In a paper by Alday, Gaiotto and Tachikawa [13], the partition function of a family of $\mathcal{T}_{g, n}$ theories on $S^{4}$ was identified with a Liouville conformal field theory correlation function on the associated Riemann surface $C_{g, n}$

$$
\mathcal{Z}_{\mathcal{T}_{g, n}}=\left\langle\prod_{a=1}^{n} V_{m_{a}}\right\rangle_{C_{g, n}},
$$

where $V_{m_{a}}$ are vertex operators in Liouville theory associated with the punctures on the Riemann surface. ${ }^{4}$ This observation points towards an intriguing connection between four dimensional $\mathcal{N}=2$ gauge theories and the rich subject of two dimensional conformal field theory. The identification was made in a particular choice of duality frame of $\mathcal{T}_{g, n}$,

\footnotetext{
${ }^{2}$ This construction generalizes to higher rank gauge groups. This requires enriching the types of punctures on the Riemann surface. See [8] for more details.

${ }^{3}$ For the case of the four punctured sphere, denoted by $C_{0,4}$, there are three boundaries corresponding to sewing the surface along the $s$-,t- and $u$-channels.

${ }^{4} \mathrm{As}$ we review in section $2, m$ is related to the mass of a hypermultiplet.
} 
corresponding to a particular sewing of the Riemann surface from pairs of pants. The modular bootstrap of Liouville conformal field theory correlators [14-16], which associates to a correlator a different representation for each choice of sewing of the Riemann surface, can then be used to demonstrate that the partition function $\mathcal{Z}_{\mathcal{T}_{g, n}}$ is invariant under the corresponding duality group $\Gamma\left(\mathcal{T}_{g, n}\right)$. This provides strong evidence for the action of Sduality for this class of $\mathcal{N}=2$ gauge theories.

Since the supersymmetries imposed in the localization procedure are preserved by a supersymmetric circular Wilson loop $W_{R}$ in a representation $R$ of the $\mathcal{N}=2$ gauge theory, Pestun's computation also yields the exact result for the expectation value of a Wilson loop [10]

$$
\left\langle W_{R}\right\rangle=\int[d a] \operatorname{Tr}_{R} e^{2 \pi i a} \bar{Z}_{\text {Nekrasov }} Z_{\text {Nekrasov }},
$$

where $a$ denotes the scalar components of the $\mathcal{N}=2$ vector multiplets in the gauge theory. By inserting the trivial Wilson loop, one may recover the expression of the partition function $\mathcal{Z}_{\mathcal{T}_{g, n}}$.

Loop operators in gauge theories play a pivotal rôle in understanding dualities. Since they are characterized by a set of electric and magnetic charges, the action of S-duality - being a non-abelian extension of electric-magnetic duality — naturally acts on them. The study of Wilson [10, 17-20] and 't Hooft loop operators [21-24] in $\mathcal{N}=4$ super YangMills has yielded quantitative evidence in favour of the S-duality conjecture, demonstrating that the correlation function of Wilson loops and 't Hooft loops are exchanged under Sduality $[23,24] .{ }^{5}$ Characterizing loop operators in $\mathcal{N}=2$ gauge theories and computing their expectation values provides a theoretical framework in which to study S-duality for this class of theories.

In [25] a complete classification of loop operators in the $\mathcal{T}_{g, n}$ theories was performed. It was shown that loop operators in $\mathcal{T}_{g, n}$ are geometrically in one-to-one correspondence with homotopy classes of non-self-intersecting curves on the Riemann surface $C_{g, n}$. The charges of a loop operator, which can be labeled by a vector $d$ in a given duality frame, were identified with the Dehn-Thurston data $d$ of a closed curve $\gamma_{d}$ on the Riemann surface $C_{g, n}$ in the corresponding choice of pants decomposition.

In this paper we identify loop operators $L_{d}$ in the $\mathcal{N}=2$ gauge theory $\mathcal{T}_{g, n}$ with loop operators $\mathcal{L}\left(\gamma_{d}\right)$ we construct in the Liouville conformal field theory on $C_{g, n}$. Our proposal gives the following Liouville conformal field theory realization for the expectation value of a gauge theory loop operator

$$
\left\langle L_{d}\right\rangle_{\mathcal{T}_{g, n}}=\left\langle\prod_{a=1}^{n} V_{m_{a}} \cdot \mathcal{L}\left(\gamma_{d}\right)\right\rangle_{C_{g, n}} .
$$

The identification of loop operators in $\mathcal{N}=2$ gauge theories with two dimensional conformal field theory correlation functions, introduces a theoretical framework in which to compute exactly the expectation value of loop operators in certain $\mathcal{N}=2$ gauge theories.

The modular properties of the Liouville loop operators combined with modular invariance of Liouville correlation functions [14-16] implies that the correlator on the right hand

\footnotetext{
${ }^{5}$ The explicit comparison was performed to next to leading order in the coupling constant expansion.
} 
side of (1.4) is invariant under a change of pants decomposition of the Riemann surface $C_{g, n}$. In contrast, the precise identification of the loop operator $L_{d}$ on the left hand side of (1.4) - whether it is a Wilson, 't Hooft or dyonic operator - depends very sensitively on the choice of duality frame of $\mathcal{T}_{g, n}$. We will show that, given a curve $\gamma_{d}$ in $C_{g, n}$, there always exists a choice of pants decomposition $\sigma_{W}$ such that the result of the Liouville correlator exactly reproduces Pestun's computation for the Wilson loop expectation value (1.3)

$$
\left\langle W_{R}\right\rangle_{\mathcal{T}_{g, n}}=\left\langle\prod_{a=1}^{n} V_{m_{a}} \cdot \mathcal{L}\left(\gamma_{d}\right)\right\rangle_{C_{g, n}} .
$$

This fits rather nicely with the field theory result stating that any dyonic operator in the $\mathcal{N}=2$ theories $\mathcal{T}_{g, n}$ can always be mapped to a Wilson loop by the action of S-duality [25, 26]. In other duality frames, the Liouville correlator (1.4) computes the expectation value of a dyonic operator.

The modular invariance of Liouville correlation functions in the presence of loop operators, together with our proposal (1.4) implies for the gauge theory observables that

$$
\left\langle L_{d, \sigma}\right\rangle_{\mathcal{T}_{g, n}}=\left\langle L_{d^{\prime}, \sigma^{\prime}}\right\rangle_{\mathcal{T}_{g, n}},
$$

where $d$ and $d^{\prime}$ are the charges of the loop operator in the $\mathcal{T}_{g, n}$ duality frames $\sigma$ and $\sigma^{\prime}$ respectively. Therefore, our proposal automatically incorporates the conjectured action of the S-duality group $\Gamma\left(\mathcal{T}_{g, n}\right)$ on gauge theory loop operators.

The quantization of the Teichmüller space of the Riemann surface $C_{g, n}$, referred to as quantum Teichmüller theory, provides a dual description of Liouville theory leading to a geometric description of loop operators in $\mathcal{T}_{g, n}$. In recent years, a precise correspondence between Liouville conformal field theory and quantum Teichmüller theory has been established [27-29]. ${ }^{6}$ We will show that the loop operators $\mathcal{L}(\gamma)$ are in one-to-one correspondence with the operators $\mathrm{L}_{\gamma}$ representing the geodesic length functions in the quantum theory of the Teichmüller spaces, and that the expectation values (1.4) are equal to the expectation values

$$
\left\langle q\left|\mathrm{~L}_{\gamma}\right| q\right\rangle,
$$

with $|q\rangle$ being the coherent state associated to the complex structure of the surface $C_{g, n}$ in quantum Teichmüller theory.

It follows that the algebra of length operators, which has been well-studied in the literature, should be identified with the algebra of loop operators in gauge theory. We show as a particular consequence that the gauge theory loop operators captured by Liouville theory satisfy the 't Hooft commutation relations, which are known to be obeyed by gauge theory loop operators that are Hopf-linked in a constant time slice. We also demonstrate that the charges of the operators that appear in the OPE, as determined by the algebra of length operators, precisely matches the prediction of S-duality, thus providing a non-trivial consistency check of our proposal.

The plan of the rest of the paper is as follows. In section 2 we briefly introduce the necessary ingredients entering the construction of the $\mathcal{N}=2$ gauge theories $\mathcal{T}_{\text {g,n }}$ given a

\footnotetext{
${ }^{6}$ The existence of such a correspondence had been predicted in [30].
} 
Riemann surface $C_{g, n}[8]$ and the relation to Liouville theory found in [13]. We then recall the classification of loop operators in $\mathcal{T}_{g, n}$ and identification of loop operators with nonself-intersecting closed curves in $\gamma$. In section 3 we introduce the notion of loop operators in Liouville field theory, construct them in terms of the Lax connection and relate the holonomy of the Lax connection around a closed curve with the monodromy of a degenerate operator in the Liouville conformal field theory as it goes around the curve.

In section 4 we make the explicit identification relating loop operators in gauge theory with loop operators in Liouville field theory. We put forth an exact prescription for calculating the monodromies of degenerate operators and compare the result to the known gauge theory expression for the Wilson loop. In section 5 we implement this general prescription, first by providing the necessary tools and then study specific examples of Wilson loops and 't Hooft loops in $\mathcal{N}=2^{*}$ theory and $\mathcal{N}=2 \mathrm{SU}(2)$ gauge theory with four fundamental flavours

A more abstract point of view on the problem is presented in section 6 which reviews quantum-Teichmüller theory and the realization of loop operators as geodesic length operators. Section 7 uses the algebra of geodesic length operators to study the algebra of gauge theory loop operators, and compares it to the explicit expressions we derived for the loop operators in section 5 .

We conclude with a discussion of our results in section 8. Some technical calculations and relevant formulae are relegated to the appendices.

Note. We have coordinated the submission to the arXiv of our work and the related work by Fernando Alday, Davide Gaiotto, Sergei Gukov, Yuji Tachikawa and Herman Verlinde [31]. We would like to thank them for arranging the joint release of the two papers.

\section{Gauge theories, Liouville theory and curves on Riemann surfaces}

In this section we review the key elements in Gaiotto's construction of the $\mathcal{N}=2$ theories $\mathcal{T}_{g, n}$ and of the connection with Liouville theory [13]. We also recount the classification of loop operators in $\mathcal{T}_{g, n}$ in terms of curves on the Riemann surface $C_{g, n}$ [25]. Once equipped with the key ingredients, we will proceed with the Liouville conformal field theory realization of the gauge theory loop operators in the following sections.

\subsection{Gauge theories and Liouville theory}

In [8], Gaiotto has put forward an algorithm which associates a four dimensional $\mathcal{N}=2$ theory to a Riemann surface. Furthermore, he has interpreted the theory as the low energy description of coincident M5-branes wrapping the Riemann surface. In this paper we consider a special class of these theories, namely, those whose gauge group is the product of several SU(2) groups. They arise from the $A_{1}(2,0)$ six dimensional conformal field theory on a genus $g$ Riemann surface with $n$ punctures $C_{g, n}$ in the presence of $n$ codimensiontwo defect operators in the $(2,0)$ theory. The theory associated with the Riemann surface $C_{g, n}$ is denoted by $\mathcal{T}_{g, n}$. The Riemann surface $C_{g, n}$ plays a key rôle in the construction. There is a canonical double cover of $C_{g, n}$ corresponding to the Seiberg-Witten curve of 


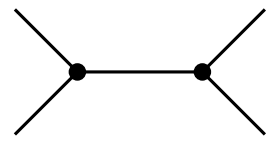

(a)

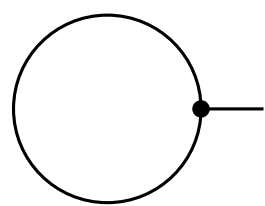

(b)

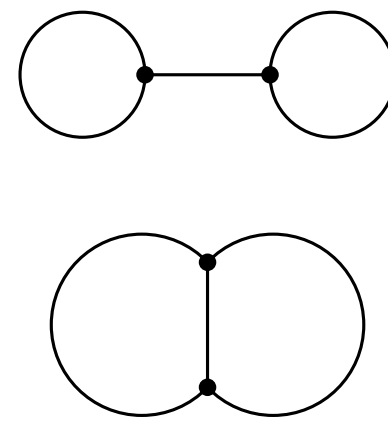

(c)

Figure 1. Examples of trivalent graphs (quiver diagrams) corresponding to Riemann surfaces: (a) a 4-puncture sphere, (b) a once-punctured torus, (c) a genus two surface with no puncture.

$\mathcal{T}_{g, n}$ which determines the prepotential of the gauge theory. The curve $C_{g, n}$ is therefore of direct relevance for the physics of the four-dimensional gauge theory $\mathcal{T}_{g, n}$.

A weakly coupled description of the $\mathcal{T}_{g, n}$ theory is encoded in a trivalent graph, also referred as a (generalized) quiver diagram. Given a choice of sewing $\sigma$ of $C_{g, n}$ from $2 g-2+n$ pairs of pants one may associate a trivalent graph $\Gamma_{\sigma}$, whose thickening spans $C_{g, n}$ in the corresponding region in Teichmüller space. ${ }^{7}$ The trivalent graph has $3 g-3+n$ internal edges and $n$ external ones. The field content of this description of the $\mathcal{N}=2 \mathcal{T}_{g, n}$ theory can be read off from the trivalent graph by associating to each internal edge an $\mathrm{SU}(2)$ gauge group and to each cubic vertex eight half-hypermultiplets in the trifundamental fundamental representation of the $\mathrm{SU}(2)^{3}$ group associated to the three incoming edges. When the edge is external the $\mathrm{SU}(2)$ symmetry corresponds to a global symmetry. Different choices of pants decomposition of $C_{g, n}$ give rise to different weakly coupled descriptions of $\mathcal{T}_{g, n}$.

The two simplest theories of this class are $\mathcal{T}_{1,1}$ and $\mathcal{T}_{0,4}$. The first case, $\mathcal{T}_{1,1}$ corresponds to $\mathcal{N}=2^{*}$ super Yang-Mills, the mass deformation of $\mathcal{N}=4$ super Yang-Mills. The relevant Riemann surface $C_{1,1}$ is the one-punctured torus whose pants decomposition involves only a single pair of pants with two legs glued to each other. A quiver diagram for this theory is shown in figure $1 \mathrm{~b}$. The $\mathcal{T}_{0,4}$ theory also has a single gauge group and it has four fundamental hypermultiplets. The corresponding surface is $C_{0,4}$ - the four-punctured sphere - which can be decomposed into two pairs of pants glued at a single leg. This leads to the quiver diagram in figure 1a.

The Riemann surface $C_{g, n}$ encodes more information than the field content of the gauge theory $\mathcal{T}_{g, n}$. The space of couplings of the gauge theory is identified with the Teichmüller space of $C_{g, n}$, i.e., the space of deformations of the complex structure of $C_{g, n}$ through the

\footnotetext{
${ }^{7}$ More precisely, in order to fully specify the duality frame in which the weakly coupled description is valid, we need to specify both a trivalent graph $\Gamma_{\sigma}$ and a pants decomposition. The pair of these data is called a "marking" (see e.g., [29]). In this paper $\sigma$ really denotes a marking, but we will often refer to it by the more familiar term "pants decomposition".
} 
formula

$$
q_{i}=\exp \left(2 \pi i \tau_{i}\right) \quad i=1, \ldots, 3 g-3+n
$$

where

$$
\tau_{i} \equiv \frac{\theta_{i}}{2 \pi}+\frac{4 \pi i}{g_{i}^{2}}
$$

are the gauge coupling constants and $q_{i}$ are the gluing parameters associated with the pants decomposition $\sigma$ of $C_{g, n}$. Thus the physical parameter space of the gauge theory is precisely the moduli space of $C_{g, n}$, obtained by modding the Teichmüller space by $\operatorname{MCG}\left(C_{g, n}\right)=$ $\Gamma\left(\mathcal{T}_{g, n}\right)$. Furthermore, the duality group $\Gamma\left(\mathcal{T}_{g, n}\right)$ acting on $\mathcal{T}_{g, n}$ has an elegant geometrical interpretation as the mapping class group $\operatorname{MCG}\left(C_{g, n}\right)$ of $C_{g, n}$.

In [13] the correspondence between a four dimensional $\mathcal{N}=2$ gauge theory and a Riemann surface was extended to a correspondence between the four dimensional gauge theory and the Liouville conformal field theory living on the Riemann surface.

The action describing Liouville field theory on an arbitrary genus $g$ Riemann surface with $n$ punctures - henceforth denoted by $C_{g, n}$ - is given by

$$
S=\frac{1}{4 \pi} \int d^{2} z\left(g^{a b} \partial_{a} \phi \partial_{b} \phi+Q R \phi+4 \pi \mu e^{2 b \phi}\right),
$$

where $(z, \bar{z})$ is a local coordinate system on the Riemann surface, $Q=b+1 / b$ and the Liouville central charge is $c=1+6 Q^{2}$ (See reference [14] for a review of Liouville field theory). The operators creating delta function normalizable states are labeled by a quantum number $\alpha \in Q / 2+i \mathbb{R}^{+}$, and give rise to a continuous spectrum. These operators, which are denoted by $V_{\alpha}$, describe the quantization of the semiclassical expression

$$
V_{\alpha}(z, \bar{z}) \simeq e^{2 \alpha \phi(z, \bar{z})}
$$

and have conformal dimension $\Delta(\alpha)=\alpha(Q-\alpha)$.

Within the conformal bootstrap approach [32], any correlation function can be constructed by "sewing" three point functions according to the pattern given by a pants decomposition of the Riemann surface $C_{g, n}$, where "sewing" is defined by summing over the vertex operator insertions representing a complete set of intermediate states. The summation splits into the integration over the different Virasoro primary fields, and the summation over their descendants. The result of the summation over the descendants factorizes into a holomorphic and an anti-holomorphic part, the so-called conformal blocks. For the correlation function (1.2), we denote the conformal block [32] in the sewing of $C_{g, n}$ given by $\sigma$ by

$$
\mathcal{F}_{\alpha, E}^{(\sigma)},
$$

where $\alpha \equiv\left(\alpha_{1}, \ldots, \alpha_{3 g-3+n}\right)$ are the labels of the representations associated to the internal edges of $\Gamma_{\sigma}$ while $E \equiv\left(m_{1}, \ldots, m_{n}\right)$ label the external vertex operator insertions.

In this formulation the correlation function of Liouville theory is written as the integral over the holomorphic and anti-holomorphic conformal blocks

$$
\left\langle\prod_{a=1}^{n} V_{m_{a}}\right\rangle_{C_{g, n}}=\int d \nu(\alpha) \overline{\mathcal{F}}_{\alpha, E}^{(\sigma)} \mathcal{F}_{\alpha, E}^{(\sigma)},
$$


where the measure $\nu(\alpha)$ includes for each pair of pants the three point functions of the primary fields, for which the explicit expression was proposed in [33, 34], and derived in $[15]$.

The conformal blocks $\mathcal{F}_{\alpha, E}^{(\sigma)}$ depend on the choice of pants decomposition $\sigma$, but the complete correlator does not. This is a consequence of the modular properties in two dimensional conformal field theory. The conformal blocks corresponding to two different trivalent graphs $\Gamma_{\sigma}$ and $\Gamma_{\sigma}^{\prime}$ are related by a change of basis, as proved in [15] for $g=0$.

A key ingredient in the identification between $\mathcal{T}_{g, n}$ and Liouville theory is played by the Nekrasov partition function $Z_{\text {instanton }}[11,12]$. This partition function, which is defined by localizing the $\mathcal{N}=2$ gauge theory with respect to a $\mathrm{U}(1) \times \mathrm{U}(1)$ subgroup of the $\mathrm{SO}(4)$ rotation group with rotation parameters $\left(\epsilon_{1}, \epsilon_{2}\right)$, is the product of the classical contribution $Z_{\text {classical }}$, the perturbative one loop contribution $Z_{1 \text {-loop }}$ and the instanton contribution $Z_{\text {instanton }}$

$$
Z_{\text {Nekrasov }}=Z_{\text {classical }} Z_{1 \text {-loop }} Z_{\text {inst }} \text {. }
$$

Given a choice of pants decomposition $\sigma$ of the Riemann surface $C_{g, n}$, the equivariant instanton partition function $[11,12]$ of the gauge theory $\mathcal{T}_{g, n}$ associated with the generalized quiver diagram $\Gamma_{\sigma}$ equals the BPZ conformal block [32] on $C_{g, n}$ in the choice of pants decomposition $\sigma[13]^{8}$

$$
Z_{\text {instanton }}^{(\sigma)}=\mathcal{F}_{\alpha, E}^{(\sigma)} .
$$

The partition function $Z_{\text {instanton }}$ depends on the zero mode of the $3 g-3+n$ scalar fields in the $\mathcal{N}=2$ vector multiplets ${ }^{9} \vec{a}$, on $n$ mass parameters $\overrightarrow{\hat{m}}$ and on the equivariant localization parameters $\epsilon_{1}$ and $\epsilon_{2}$. The BPZ conformal block [32] depends on the conformal dimension of the vertex operators decorating the $3 g-3+n$ internal edges and $n$ external edges of $\Gamma_{\sigma}$, which carry Liouville momenta $\vec{\alpha}$ and $\vec{m}$ respectively, as well as on the Liouville central charge $c$. With the proposed dictionary

$$
\begin{array}{rlr}
\alpha_{i} & =Q / 2+a_{i} \quad i=1, \ldots, 3 g-3+n \\
m_{a} & =Q / 2+\hat{m}_{a} \quad a=1, \ldots, n \\
b & =\epsilon_{1} & \\
1 / b & =\epsilon_{2}, &
\end{array}
$$

the relation (2.8) was demonstrated up to several orders in a Taylor series expansion in $q_{i}=\exp \left(2 \pi i \tau_{i}\right)[13] .^{10}$

The complete Liouville theory correlator on $C_{g, n}$ also admits an elegant gauge theory interpretation. It was found in [13] that the Liouville correlator on $C_{g, n}(1.2)$ for $b=1$ (or

\footnotetext{
${ }^{8}$ The definition of a conformal block also requires specifying how the graph $\Gamma_{\sigma}$ is drawn on the decomposed Riemann surface. See footnote 7 .

${ }^{9}$ In the gauge theory the parameters $a_{i}$ are traceless anti-hermitean matrices, and $\hat{m}_{a}$ imaginary numbers, of the same magnitude as the physical mass. Up to $\mathrm{SU}(2)$ transformations $a_{i}$ is given by one of the two eigenvalues and we identify the matrix and its eigenvalue. In (2.9) $a_{i}$ is the eigenvalue and in (1.3) and (2.10) it is a matrix with the appropriate Haar measure.

${ }^{10}$ The correspondence between the instanton partition in gauge theory and Liouville theory has been extended to a class of asymptotically free theories in [35].
} 
equivalently $c=25$ ) corresponds to the partition function of $\mathcal{T}_{g, n}$ on $S^{4}$ computed in the duality frame $\sigma[10]^{11}$

$$
\mathcal{Z}_{\mathcal{T}_{g, n}}^{(\sigma)}=\int[d a] \bar{Z}_{\text {Nekrasov }}^{(\sigma)} Z_{\text {Nekrasov }}^{(\sigma)}=\left\langle\prod_{a=1}^{n} V_{m_{a}}\right\rangle_{C_{g, n}} .
$$

Pestun's partition function resembles the holomorphically factorized representation of two dimensional conformal field theory correlators. Formula (2.10) makes this observation precise. In making the identification, the authors of [13] noted that the product of three point functions of primary operators in Liouville [15, 33, 34] precisely assemble themselves to realize the measure in Pestun's partition function (2.10).

It is important to note that the Liouville correlator $\left\langle\prod_{a=1}^{n} V_{m_{a}}\right\rangle$ does not depend on the choice of pants decomposition of $C_{g, n}$. Correlators in Liouville theory are invariant with respect to the action of $\operatorname{MCG}\left(C_{g, n}\right)$, which relate different sewings of the surface [1416]. This implies that the partition function of $\mathcal{T}_{g, n}$ on $S^{4}$ is invariant under the S-duality group $\Gamma\left(\mathcal{T}_{g, n}\right)$

$$
\mathcal{Z}_{\mathcal{T}_{g, n}}^{(\sigma)}=\mathcal{Z}_{\mathcal{T}_{g, n}}^{\left(\sigma^{\prime}\right)}
$$

where $\sigma$ and $\sigma^{\prime}$ denotes a choice of pants decomposition of $C_{g, n}$.

\subsection{Loop operators in gauge theory and curves on Riemann surfaces}

The $(2,0)$ theory in six dimensions has surface observables (strings), which arise in Mtheory from M2-branes ending on the M5-branes whose low energy dynamics are governed by this conformal field theory. Upon dimensional reduction, these observables become local operators, loop operators or surface operators, depending on how many of the dimensions are along the compact manifold and how many in the four dimensional space. In this paper we focus on the case where one of the directions is on the Riemann surface and the other is in the four dimensional space.

As far as the four dimensional theory is concerned, these are operators supported on one dimensional curves, like Wilson loops. In fact in the present framework we can study Wilson loops, 't Hooft loops and dyonic loop operators in a unified fashion. We focus on loop operators supported on a circle, which have the potential of preserving the most supersymmetries, and study the possible shapes they can take on the internal manifold.

One would therefore expect that the classification of maximally supersymmetric loop operators in the $\mathcal{N}=2$ gauge theory $\mathcal{T}_{g, n}$ is related to the classification of loop operators in Liouville theory on the two dimensional surface $C_{g, n}$. Moreover, as mentioned above, the S-duality symmetries of the gauge theory are the modular symmetries of the Riemann surface. Understanding the duality map for loop operators in Liouville theory on $C_{g, n}$ will teach us the precise working of S-duality for gauge theory loop operators, generalizing the exchange of a fundamental Wilson loop and an 't Hooft loop in $\mathcal{N}=4$ super Yang-Mills.

The classification of loop operators in these gauge theories and the mapping to curves on Riemann surfaces, as well as their transformation rules under S-duality were presented in [25], as we review now.

\footnotetext{
${ }^{11}$ The precise agreement holds after a overall normalization constant for the vertex operators is removed.
} 
Loop operators in the gauge theory measure the response of the system to an external particle which may be charged both electrically and magnetically under any of the gauge groups. This gives the set of integers $\left(p_{i}, q_{i}\right)$, where $i=1, \ldots, 3 g-3+n$, with $p_{i}$ being the magnetic charge ${ }^{12}$ in the $i^{\text {th }}$ group and $q_{i}$ the electric charge. ${ }^{13}$ Some extra conditions are imposed on the set of magnetic charges $p_{i}$, due to the presence of a Dirac string in a monopole background. In order for the loop operator to be well-defined, all of the fields of the theory, in particular the hypermultiplets in fundamental representations of different $\mathrm{SU}(2)$ factors, should be single-valued even around the Dirac string. This implies that for each trivalent vertex in the quiver diagram, the sum of the three magnetic charges associated with the edges connected to it is even:

$$
p_{i}+p_{j}+p_{k} \in 2 \mathbb{Z} \text { if } i, j, k \text { are three boundaries of a pair of pants. }
$$

Beyond that, in each gauge group there is an unbroken $\mathbb{Z}_{2}$ Weyl symmetry which we have to mod out by, and therefore we may assume that $p_{i} \geq 0$, and if $p_{i}=0$ that $q_{i} \geq 0$.

This set of data should match the classification of loop operators on the surface $C_{g, n}$, or more precisely loop operators for the Liouville theory on this Riemann surface. At the purely topological level the observation of [25] is that there is a one-to-one map between the possible sets of charges of loop operators in $\mathcal{T}_{g, n}$ and homotopy classes of curves, not necessarily connected, ${ }^{14}$ without self-intersections.

There is a beautiful classification of all such curves due to Dehn [36] and independently to Thurston [37], in terms of precisely the same data

$$
d \equiv\left(p_{i}, q_{i}\right)_{i=1, \ldots, 3 g-3+n}
$$

called Dehn-Thurston parameters, as those which label the charges of gauge theory loop operators. The classification is performed for a given pants decomposition of the surface and therefore is perfectly suited to our purposes of matching with the gauge theory data in a given duality frame.

For a given pants decomposition one can choose open segments on the three boundaries of each pair of pants. By a homotopy it is possible to arrange that all the components of the curve going through the pair of pants cross the boundaries through these segments. Then each component will follow one of the six possible paths illustrated in figure 5 (the open segments are the upper halves of each of the circles). How many times the curve crosses each boundary of the pair of pants uniquely specifies the combination of paths that the curve contains. See [25] for more detailed explanation.

Clearly the total number of times the curve passes the boundaries of the pair of pants is even, matching the Dirac condition on the magnetic charges $p_{i}$, which are also all nonnegative. In gluing two pairs of pants there is a canonical identification of the curves on

\footnotetext{
${ }^{12}$ In [25] a generalization was presented which allowed for magnetic charges on the flavor groups, but we will suppress this possibility in the present paper.

${ }^{13}$ It should be clear from the context whether $q_{i}$ refers to the electric charge or the gluing parameter/modulus $\exp \left(2 \pi i \tau_{i}\right)$.

${ }^{14}$ In this section, we mean by a "curve" a one-dimensional submanifold that may have more than one connected component. In the rest of the paper, a "curve" is assumed to be connected.
} 
the two sides due to the open segments, but one may choose to do a cyclic permutation (in other words a twist around the boundary) on the lines before the identification, which can then be associated to $q_{i} \in \mathbb{Z}$. Lastly when $p_{i}=0$, there are no lines crossing the boundary between two pairs of pants, and it is possible to introduce $q_{i} \geq 0$ loops around the boundary as components of the curve such that there is no intersection among components. Thus we have a natural map between free homotopy classes $^{15}$ of non-intersecting closed curves on $C_{g, n}$ and the charges of loop operators in the gauge theory $\mathcal{T}_{g, n}$.

In particular the simple connected curve corresponding to the $i^{\text {th }}$ edge of the trivalent graph represents the purely electric loop in the $i^{\text {th }}$ group - a Wilson loop. It is also important for us to note that for any non-self-intersecting curve, there is a choice of pants decomposition such that it is homotopic to the boundary of a pair of pants. Thus any loop operator in the gauge theory corresponding to such a curve is a Wilson loop in some duality frame.

The discussion above completely classifies the allowed set of charges carried by the loop operators. Let us comment, though, that it does not specify fully what the operator is, as a Wilson loop may be in the irreducible representation of that dimension or a product of lower irreducible representations and similar refinements should apply also to 't Hooft and dyonic loops. Indeed as we shall see below, in Liouville theory it is also possible to associate different loop operators to the same set of Dehn-Thurston data. The distinction between them is simply different choices of bases for loop operators. For Wilson loop operators, where the classification of loop operators in the gauge theory is complete, we have an exact map between different bases in the gauge theory and in Liouville. ${ }^{16}$

For the most part we will concentrate below on the basis that mirrors the discussion above and which is presented in the next section. We will comment about other bases and their possible advantages in section 5.5.

\section{Holonomy and monodromy in Liouville theory}

In this section we introduce loop operators in classical Liouville theory and relate them to the monodromy of a special class of operators in Liouville theory, the so called degenerate operators. In the next section we will explain how to calculate the action of the Liouville loop operators on conformal blocks in quantum Liouville theory, and use these operators to calculate the expectation value of loop operators in $\mathcal{N}=2$ gauge theories.

The classical equations of motion of the Liouville field theory can be recast as the zero curvature representation of a connection ${ }^{17}$

$$
\mathcal{F}=d \mathcal{A}+\mathcal{A} \wedge \mathcal{A}=0 \quad \Longrightarrow \quad \text { Equations of motion }
$$

\footnotetext{
${ }^{15} \mathrm{~A}$ free homotopy class is the set of closed curves that are related by a homotopy, without the condition that the homotopy fixes a base point. The set of free homotopy classes coincides with the set of conjugacy classes of the fundamental group.

${ }^{16}$ There does not exist to date a complete classification of 't Hooft and dyonic loop operators in these gauge theories which addresses these distinctions, so we can at best identify them by their charges.

${ }^{17}$ The Liouville equation of motion on an arbitrary background metric $g$ determines the Weyl factor $e^{2 b \phi}$ which renders the curvature of the metric $\hat{g}_{a b}=e^{2 b \phi} g_{a b}$ constant and negative.
} 
The flatness of the connection on the Riemann surface arises from consistency of the following system of linear equations, known as the Lax pair

$$
\begin{aligned}
& \frac{\partial \Psi}{\partial z}+\mathcal{A}_{z} \Psi=0, \\
& \frac{\partial \Psi}{\partial \bar{z}}+\mathcal{A}_{\bar{z}} \Psi=0 .
\end{aligned}
$$

This system of equations implies that $\Psi$ is covariantly constant under parallel transport with respect to the Lax connection $\mathcal{A}$ on the Riemann surface $C_{g, n}$

$$
(d+\mathcal{A}) \Psi=0 .
$$

The chiral quantum fields associated to the classical auxiliary fields $\Psi$ represent building blocks in the construction of Liouville primary fields, and will play a key rôle in our Liouville realization of loop operators in $\mathcal{N}=2$ gauge theories with $\mathrm{SU}(2)$ gauge groups.

For the Liouville field theory, the Lax connection $\mathcal{A}$ takes values in the Lie algebra $\mathrm{SL}(2, \mathbb{R})$. The Lax connection can be recast in terms of the Liouville field $\phi$ through

$$
\mathcal{A}=e T^{+}+\bar{e} T^{-}+w T^{3},
$$

where $e$ and $w$ are the vielbein and spin connection of the two dimensional metric on the Riemann surface $C_{g, n}$ given by $\hat{g}_{a b}=\exp (2 b \phi) g_{a b}$, which has constant negative curvature. $\left\{T^{3}, T^{+}, T^{-}\right\}$are $\mathrm{SL}(2, \mathbb{R})$ Lie algebra generators in the $j=1 / 2$ representation.

The existence of an $\mathrm{SL}(2, \mathbb{R})$ Lax connection in Liouville field theory allows us to parallel transport the fields $\Psi$ between any two points $x$ and $y$ on the Riemann surface $C_{g, n}$. Since the Lax connection is flat, the holonomy depends only on the homotopy class of the path connecting $x$ and $y$, and allows us to relate fields at different points on $C_{g, n}$

$$
\Psi(x)=U(x, y) \Psi(y), \quad U(x, y)=\mathrm{P} \exp \int_{x}^{y} \mathcal{A} .
$$

Of particular importance is the holonomy obtained by integrating the Lax connection around an arbitrary non-self-intersecting closed loop $\gamma$ on the Riemann surface $C_{g, n}$

$$
U(\gamma)=\mathrm{P} \exp \oint_{\gamma} \mathcal{A}
$$

In particular, the holonomy around a closed curve encircling a tube in the pants decomposition of $C_{g, n}$ is obtained by integrating

$$
\mathcal{A}_{x}=\left(\begin{array}{cc}
b \partial_{t} \phi & \mu e^{b \phi} \\
\mu e^{b \phi} & -b \partial_{t} \phi
\end{array}\right)
$$

around the circle parametrized by $x$, where $(t, x)$ are coordinates on the tube. Heuristically, the holonomy around the tube measures the momentum of the state propagating across it. The connection (3.7) is gauge equivalent to

$$
\mathcal{A}_{x}=\left(\begin{array}{cc}
0 & -b^{2} T \\
1 & 0
\end{array}\right),
$$


where $T$ is the Liouville stress tensor. This form will be useful when relating the auxiliary fields $\Psi$ with degenerate fields.

The discussion has thus far been restricted to classical Liouville theory, where $b \ll 1$ (or equivalently $b \gg 1$ ). We are interested in performing the computation of the insertion of a Wilson loop in the Liouville conformal field theory correlator in the quantum regime. In particular we are interested in the case $b=1$, when the Liouville correlator corresponds to the partition function of the dual gauge theory on $S^{4}$, which is very quantum in Liouville.

It is not easy, but possible to define the Wilson loop in the quantum theory by means of a lattice regularization [38]. An alternative approach is to first quantize the chiral fields $\Psi$, and to build the Liouville field out of these building blocks later [14, 27]. The loop operator associated to the loop $\gamma$ can then be defined in terms of the monodromy of chiral fields $\Psi$. We introduce it here in the quantum theory (and define rigorously in the following section) and explain why it reduces to the holonomy in the classical limit.

We recall that the fields $V_{\alpha}$ in Liouville giving rise to delta function normalizable states are labeled by a quantum number $\alpha \in Q / 2+i \mathbb{R}$. Degenerate operators/fields, on the other hand, are labeled by a pair of integers $(r, s) \in \mathbb{Z}_{>0} \times \mathbb{Z}_{>0}$. Their corresponding quantum numbers are given by

$$
2 \alpha_{r, s}=Q-\frac{r}{b}-s b
$$

In this paper, we denote the chiral degenerate fields by $V_{r, s}$. The complete, conformal primary degenerate field is obtained by combining $V_{r, s}$ with its anti-holomorphic counterpart, but in this paper only the chiral degenerate field $V_{r, s}$ is relevant. The defining property of a degenerate field is that it contains a Virasoro descendant that is a null state (a state of zero norm). Decoupling of null states imposes strong constraints on the correlation functions of the theory. A chiral degenerate field $V_{r, s}$ contains a null state at Virasoro level $r s$, and decoupling of such a state is captured by the action of a differential operator of order $r s$ on the correlation functions of the theory containing the chiral degenerate field $V_{r, s}$. The differential operators constraining the correlation functions are of Fuchsian type and, therefore, their solutions are not single valued, rather they acquire monodromies when encircling the singularities in the differential equation.

The monodromies of the chiral degenerate fields correspond precisely to the holonomy acquired by transporting the Lax connection around a closed curve $\gamma$,

$$
\Psi(x+2 \pi)=U(\gamma) \cdot \Psi(x)
$$

where $x \in C_{g, n}$ is a base point of the curve $\gamma$ in $C_{g, n}$. This can be verified by noting that the null decoupling differential equation

$$
\left(\partial^{2}+b^{2} T(z)\right) V_{1,2}=0,
$$

is precisely the equation for parallel transport with respect to the Lax connection in the spin $1 / 2$ representation $(3.8)$. 
The relation between the differential equation associated to the $V_{1,2 j+1}$ chiral degenerate field and the parallel transport equation extends to arbitrary spin $j .{ }^{18}$ The Lax connection has a counterpart in the spin $j$ representation of $\mathrm{SL}(2, \mathbb{R})$, and the corresponding system of $2 j+1$ first order linear differential equations (3.2) for $2 j+1$ fields $\Psi_{j, m}$ can be recast in terms of a single differential equation of order $2 j+1$. This differential equation precisely coincides with the null decoupling equation for the $V_{1,2 j+1}$ chiral degenerate field in the semiclassical approximation. This identifies the chiral degenerate field $V_{1,2 j+1}$ as the quantization of the auxiliary fields $\Psi_{j, m} \cdot{ }^{19}$ This establishes the correspondence

$$
\operatorname{Tr}_{j} U(\gamma) \Longleftrightarrow \text { Monodromy of } V_{1,2 j+1} \text { degenerate field, }
$$

where $\operatorname{Tr}_{j} U(\gamma)$ is a Wilson loop in the spin $j$ representation of $\operatorname{SL}(2, \mathbb{R})$.

While we perform our calculations for arbitrary values of $b$, we eventually focus our analysis to $b=1$, for which a gauge theory interpretation of the Liouville correlators is available. Inspection of (3.9) shows that there is a large redundancy in the labeling of degenerate fields for $b=1$, as they depend actually only on the sum $r+s$. Without loss of generality, we have chosen a basis of chiral degenerate fields spanned by $V_{1,2 j+1}$.

There are different types of Wilson loops that can be inserted into the Liouville conformal field theory correlator, depending upon whether the Wilson loop along the curve $\gamma$ is in the spin $j$ representation, multiply wound or multi-traced. The corresponding statement in terms of degenerate fields is

$$
\begin{aligned}
\operatorname{Tr}_{j} U(\gamma) & \Longleftrightarrow \quad \text { Monodromy of } V_{1,2 j+1} \text { along } \gamma \\
\operatorname{Tr}\left[U(\gamma)^{k}\right] & \Longleftrightarrow \quad \text { Monodromy of } V_{1,2} \text { along } k \cdot \gamma \\
{[\operatorname{Tr} U(\gamma)]^{k} } & \Longleftrightarrow \quad k \text { times the Monodromy of } V_{1,2} \text { along } \gamma
\end{aligned}
$$

One point that is worth mentioning is that based on the analysis of [25] we focus our discussion on non-intersecting curves, ${ }^{20}$ even though there is no impediment to considering the classical holonomy or a monodromy along curves with intersections. There are two justifications for that. First based on the comparison with the gauge theory in [25], we expect non-intersecting loops to provide a complete basis for loop operators. Moreover intersecting curves lead to more severe divergences than smooth curves and require more care to regularize in the quantum theory.

We expect the resolution of these singularities to invoke mixing between different degenerate fields, which we comment on in section 5.5. We also address the issue of intersecting curves in section 7 , where we indeed show that they can be represented as linear combinations of non-intersecting ones.

\footnotetext{
${ }^{18} \mathrm{As}$ another example of this correspondence, the degenerate field $V_{1,3}$ satisfies the semiclassical null decoupling equation $\left(\partial^{3}+4 b^{2} T+2 b^{2} \partial T\right) V_{1,3}=0$, which translates into flatness of the Lax connection in the $j=1$ representation.

${ }^{19}$ If, in particular, we are considering the complete, conformal primary degenerate field with label $(1,2 j+1)$, it turns out to be possible to reconstruct this degenerate field from the $2 j+1$ linearly independent solutions $\Psi_{j, m}(z), m=-j, \ldots, j$ of the null vector decoupling equations and their anti-holomorphic counterparts $\bar{\Psi}_{j, m}(\bar{z})$.

${ }^{20}$ Note though that when resolved, the multi-wound loop $\operatorname{Tr} U^{k}$ has intersections while $(\operatorname{Tr} U)^{k}$ does not.
} 
In the next section we make the discussion of the monodromy more precise and provide the explicit connection to the gauge theory loop operators.

\section{Loop operators in gauge theories from Liouville theory}

In this section we give an exact prescription for calculating the monodromy associated to transporting a degenerate field along a simple connected curve $\gamma$ on the Riemann surface by introducing the notion of a quantum loop operator $\mathcal{L}(\gamma)$ associated with the closed curve. In a duality frame where $\gamma$ represents a Wilson loop, the definition we present exactly reproduces the result of Pestun (1.3) and for all other loops it provides a prediction for the gauge theory observable. In the next section we follow this procedure in some simple examples.

\subsection{Loop operators in Liouville theory}

Our goal is to study the effect of inserting the monodromy of a degenerate field into the Liouville conformal field theory correlator $\left\langle\prod_{a=1}^{n} V_{m_{a}}\right\rangle_{C_{g, n}}$. We perform the calculations using the conformal bootstrap approach as reviewed in section 2. We first define the calculational procedure somewhat abstractly and then spell it out in explicit detail.

In order to calculate the monodromy of a degenerate field, we must first think about enriching a conformal block with extra punctures corresponding to the degenerate fields in question. Consider the simplest situation where two degenerate fields of type $V_{1,2}$ are used $^{21}$ (the reason why two fields rather than one will become apparent shortly). We denote the space of conformal blocks on $C_{g, n}$ by $\mathcal{C}\left(C_{g, n}\right)$ and those on $C_{g, n+2}$ when two extra punctures are of type $V_{1,2}$ by $\hat{\mathcal{C}}\left(C_{g, n+2}\right)$,

An important property of degenerate fields is that their OPE with a Liouville primary operator truncates

$$
\left[V_{1,2}\right] \cdot\left[V_{\alpha}\right]=\left[V_{\alpha+b / 2}\right]+\left[V_{\alpha-b / 2}\right] .
$$

This equation implies that $\hat{\mathcal{C}}\left(C_{g, n+2}\right)$ is isomorphic to $\mathcal{C}\left(C_{g, n}\right) \otimes \mathbb{C}^{4}$ as a vector space. The key observation to be made is that there is an embedding

$$
\imath_{g, n}: \mathcal{C}\left(C_{g, n}\right) \hookrightarrow \hat{\mathcal{C}}\left(C_{g, n+2}\right),
$$

coming from the fact that the fusion of the two degenerate fields $V_{1,2}$ contains the vacuum representation.

This can be seen more explicitly as follows. Consider a pants decomposition $\hat{\sigma}$ of the Riemann surface $C_{g, n+2}$ in which both representations $V_{1,2}$ are in the same pair of pants. The OPE of these two degenerate fields contains only the identity state and the $V_{1,3}$ state. The conformal block $\hat{\mathcal{F}}_{\alpha, E}^{(\hat{\sigma})}$ associated to this pants decomposition vanishes unless the edge connecting this pair of pants to the rest of the surface carries the representation $V_{0}$ or $V_{1,3}$. The subspace spanned by the elements with the identity $V_{0}$ as the intermediate state is isomorphic to $\hat{\mathcal{C}}\left(C_{g, n+1}\right)$, with the $(n+1)^{\text {st }}$ boundary component being assigned the vacuum representation $V_{0}$. This space is canonically isomorphic to $\mathcal{C}\left(C_{g, n}\right)$.

\footnotetext{
${ }^{21}$ It is clear how the construction generalizes. One can add pairs of higher degenerate fields $V_{r, s}$.
} 
It follows from the existence of the embedding (4.2) that the mapping class group action on $\hat{\mathcal{C}}\left(C_{g, n+2}\right)$ can be projected onto $\mathcal{C}\left(C_{g, n}\right)$. The mapping class group $\operatorname{MCG}\left(C_{g, n+2}\right)$ contains in particular the monodromies generated by moving the insertion point of one of the representations $V_{1,2}$ along a connected simple closed curve $\gamma$ on $C_{g, n}$. The projection of the action of these elements on $\hat{\mathcal{C}}\left(C_{g, n+2}\right)$ down to $\mathcal{C}\left(C_{g, n}\right)$ defines operators on $\mathcal{C}\left(C_{g, n}\right)$. These are the Liouville loop operators $\mathcal{L}(\gamma)$ we are interested in.

A key property of the Liouville loop operators, which is an immediate consequence of this definition, is their modular invariance. The description above does not depend on a pants decomposition of the Riemann surface $C_{g, n}$, it only requires to separate the two extra degenerate fields on a pair of pants to get the canonical identification between $\hat{\mathcal{C}}\left(C_{g, n+2}\right)$ and $\mathcal{C}\left(C_{g, n}\right)$. Since Liouville theory is modular invariant [14-16], so are the loop operators. In the explicit calculations below we will employ specific pants decompositions for the entire surface and the details of the calculation will depend on it, but by this argument, the final result is modular invariant.

We turn now to presenting a more concrete form of the loop operators in terms of their action on the conformal blocks. Since we chose for the construction the chiral degenerate operators, the monodromy acts non-trivially only on the holomorphic conformal block (2.5) while it acts trivially on the antiholomorphic one $\mathrm{e}^{22}$

$$
\begin{aligned}
& \mathcal{F}_{\alpha, E}^{(\sigma)} \longrightarrow\left[\mathcal{L}(\gamma) \cdot \mathcal{F}^{(\sigma)}\right]_{\alpha, E} \\
& \overline{\mathcal{F}}_{\alpha, E}^{(\sigma)} \longrightarrow \overline{\mathcal{F}}_{\alpha, E}^{(\sigma)} .
\end{aligned}
$$

Once we have an explicit expression for $\mathcal{L}(\gamma)$, the Liouville correlation function (2.6) gets modified by the insertion of Liouville loop operator to

$$
\langle\mathcal{L}(\gamma)\rangle_{C_{g, n}}=\int d \nu(\alpha) \overline{\mathcal{F}}_{\alpha, E}^{(\sigma)}\left(\mathcal{L}(\gamma) \cdot \mathcal{F}_{\alpha, E}^{(\sigma)}\right)
$$

As we discussed in section 3, it is also possible to consider the monodromy of a higher degenerate field $V_{1,2 j+1}$. Clearly it is possible to defined a more general Liouville loop operator by replacing the lowest degenerate field $V_{1,2}$ by $V_{1,2 j+1}$ in the above definition of $\mathcal{L}(\gamma)$. We will denote the resulting loop operator by $\mathcal{L}_{j}(\gamma)$. In the special case $j=1 / 2$, we obtain $\mathcal{L}(\gamma) \equiv \mathcal{L}_{1 / 2}(\gamma)$ back. We will comment on different bases for loop operators which utilize $V_{1,2}$ or more general $V_{1,2 j+1}$ fields in section 5 and in section 7 . For the most part, though, we will focus on the basis mirroring the discussion in section 2.2, transporting $V_{1,2}$ along non-intersecting curves on the surface. This also corresponds to the last line in (3.13),

\subsection{Calculational scheme}

In order to explicitly calculate the action of the Liouville loop operator $\mathcal{L}(\gamma)$ on the conformal blocks $\mathcal{F}_{\alpha, E}^{(\sigma)}$ we follow the following algorithm that was originally used to derive the Verlinde formula [39]:

\footnotetext{
${ }^{22}$ One could equally well choose to act on the antiholomorphic blocks. They get acted on by the adjoint of the operators $\mathcal{D}_{\alpha}^{(\sigma)}$ defined in (4.5). The distinction completely disappears when the discussing length operators in quantum Teichmüller theory (1.7), see section 6 .
} 
1. Start by inserting the identity operator at a point in the trivalent graph which is spanned by the curve $\gamma$. The corresponding conformal blocks transform under changes of the marking exactly as $\mathcal{F}_{\alpha, E}^{(\sigma)}$.

2. We represent the identity operator as the projection to the identity of the fusion of two degenerate fields. This operation adds two external edges to the original trivalent graph $\Gamma_{\sigma}$, associated to these degenerate fields, and yields a new trivalent graph $\Gamma_{\hat{\sigma}}$. We denote the conformal block corresponding to this enriched trivalent graph by $\hat{\mathcal{F}}_{\alpha, E}^{(\hat{\sigma})}$.

3. We transport one of the degenerate fields around the trivalent graph $\Gamma_{\sigma}$ to span the curve $\gamma$, and return it to its original position. Each intermediate step involves a different trivalent graph $\Gamma_{\hat{\sigma}^{\prime}}$, where the degenerate field is connected to different edges in $\Gamma_{\sigma}$.

4. We fuse again the two degenerate fields to produce the identity operator.

Following the strategy we have described provides a concrete computational framework in which to calculate $\left\langle\mathcal{L}\left(\gamma_{d}\right)\right\rangle_{C_{g, n}}$. In order to evaluate the monodromy (specifically step 3) we must find how conformal blocks based on inequivalent trivalent graphs are related to each other. Trivalent graphs associated with a given Riemann surface $C_{g, n}$ can be transformed into each other by the action of the so-called Moore-Seiberg groupoid [40] (see [41] for a review). The Moore-Seiberg groupoid is generated by three basic moves, which act on the space of trivalent graphs. They are

- Fusion move

- Braiding move

- S-move

It is an important result in Liouville theory that the generators of the Moore-Seiberg groupoid act on the space of conformal blocks [14-16]. Once the action of the generators on the conformal blocks is known, the monodromy action on the conformal block $[\mathcal{L}(\gamma)$. $\left.\mathcal{F}^{(\sigma)}\right]_{\alpha, E}$ can be obtained by concatenating the moves that are required to create the pair of degenerate fields, move them around the trivalent graph and annihilate them again.

In section 5 we write down the expressions for the fusion and braiding moves on the Liouville conformal blocks and use them to calculate the expectation value of various loop operators.

The general fusion matrices are quite complicated, but they simplify significantly when one of the legs is a degenerate operator. Due to the OPE property of the $V_{1,2}$ degenerate field (4.1), the basis change in each step in the calculation involves a sum over two terms, of conformal blocks with representations shifted by $\pm b / 2$.

At the end of the calculation, after the degenerate fields are projected out we are back at the original basis for $\mathcal{C}\left(C_{g, n}\right)$ being generated by the conformal blocks $\mathcal{F}_{\alpha, E}^{(\sigma)}$, but the representations $\alpha$ carried by the edges in the graph $\Gamma_{\sigma}$ have been shifted by integer multiples of $-b / 2$. We conclude that the operator $\mathcal{L}(\gamma)$ can be represented as a difference 
operator $\mathcal{D}_{\alpha}^{(\sigma)}(\gamma)$ which acts by shifting the indices $\alpha$ on $\mathcal{F}_{\alpha, E}^{(\sigma)}$ and by multiplying the conformal blocks with $\alpha$ and $E$-dependent factors:

$$
\left[\mathcal{L}(\gamma) \cdot \mathcal{F}^{(\sigma)}\right]_{\alpha, E}=\mathcal{D}_{\alpha}^{(\sigma)}(\gamma) \mathcal{F}_{\alpha, E}^{(\sigma)}
$$

Within this framework one may demonstrate the modular invariance of the Liouville loop operators more explicitly as follows. The operators $\mathcal{D}_{\alpha}^{(\sigma)}(\gamma)$ were defined as projections of an operator $M_{\hat{\sigma}}(\gamma)$ on $\hat{\mathcal{C}}\left(C_{g, n+2}\right)$, which represents the element in $\operatorname{MCG}\left(C_{g, n+2}\right)$ corresponding to the monodromy associated to transporting a degenerate field along $\gamma$. We are using the notation $\hat{\sigma}$ for a pants decomposition (more precisely a marking, see footnote 8) on $C_{g, n+2}$ which becomes a pants decomposition on $C_{g, n}$ by splitting off the pair of pants containing the insertions of the two degenerate fields. Choosing another pants decomposition $\sigma^{\prime}$ would yield an operator $D_{\alpha}^{\left(\sigma^{\prime}\right)}(\gamma)$. The conformal blocks in $\hat{\mathcal{C}}\left(C_{g, n+2}\right)$ associated to $\hat{\sigma}$ and $\hat{\sigma}^{\prime}$ are related by an operator $M_{\hat{\sigma}^{\prime} \hat{\sigma}}$. The fact that the operators $M_{\hat{\sigma}^{\prime} \hat{\sigma}}$ represent the modular groupoid implies in particular the relation

$$
M_{\hat{\sigma}^{\prime} \hat{\sigma}} \cdot M_{\hat{\sigma}}(\gamma)=M_{\hat{\sigma}^{\prime}}(\gamma) \cdot M_{\hat{\sigma}^{\prime} \hat{\sigma}}
$$

Projecting this relation to $\mathcal{C}\left(C_{g, n}\right)$ yields

$$
M_{\sigma^{\prime} \sigma} \cdot \mathcal{D}_{\alpha}^{(\sigma)}(\gamma)=\mathcal{D}_{\alpha}^{\left(\sigma^{\prime}\right)}(\gamma) \cdot M_{\sigma^{\prime} \sigma}
$$

where now $M_{\sigma^{\prime} \sigma}$ relates the conformal blocks in $\mathcal{C}\left(C_{g, n}\right)$ associated to $\sigma$ and $\sigma^{\prime}$. This shows that the operators $\mathcal{D}_{\alpha}^{(\sigma)}(\gamma)$ are mapped to each other by the change of representation induced by change of pants decomposition. They therefore represent an operator $\mathcal{L}(\gamma)$ that is independent of the choice of pants decomposition on the Riemann surface.

As mentioned before, it is only the concrete realization of the operator as a difference operator acting on $\alpha$ that may change when changing the pants decomposition $\sigma$. The modular invariance of Liouville correlation functions [14-16] therefore implies the modular invariance of the Liouville loop operator expectation values. The fact that the expectation values $\langle\mathcal{L}(\gamma)\rangle_{C_{g, n}}$ defined in (4.4) are modular invariant allows us to regard the $\mathcal{L}(\gamma)$ as natural observables in Liouville theory.

\subsection{Relation to the gauge theory}

As reviewed in section 2, loop operators in $\mathcal{T}_{g, n}$ are in one-to-one correspondence with non-self-intersecting geodesics in $C_{g, n}$. Our proposal is that the expectation value of a loop operator $L_{d}$ in the $\mathcal{N}=2$ theory $\mathcal{T}_{g, n}$ is captured by inserting into the Liouville correlator the loop operator $\mathcal{L}\left(\gamma_{d}\right)$

$$
\left\langle L_{d, \sigma}\right\rangle_{\mathcal{T}_{g, n}}=\left\langle\mathcal{L}\left(\gamma_{d, \sigma}\right)\right\rangle_{C_{g, n}}
$$

We note that for all the theories $\mathcal{T}_{g, n}$, any loop operator $L_{d}$ can always be transformed into a duality frame $\sigma$ where the operator is purely electric, and corresponds to a Wilson loop in the four dimensional gauge theory. In this case the corresponding conformal block $\mathcal{F}_{\alpha, E}^{(\sigma)}$ is an eigenstate of the loop operator $\mathcal{L}(\gamma)$

$$
\left[\mathcal{L}(\gamma) \cdot \mathcal{F}^{(\sigma)}\right]_{\alpha, E}=\lambda(\alpha) \mathcal{F}_{\alpha, E}^{(\sigma)}
$$


By explicit calculation, when the monodromy is that of a $V_{1,2}$ degenerate field around the curve $\gamma$ the action is (5.15)

$$
\left[\mathcal{L}(\gamma) \cdot \mathcal{F}^{(\sigma)}\right]_{\alpha, E}=\frac{\cos (\pi b(2 \alpha-Q))}{\cos (\pi b Q)} \mathcal{F}_{\alpha, E}^{(\sigma)}
$$

Using our proposal (4.8) we find that the Liouville correlator $\left\langle\mathcal{L}\left(\gamma_{d, \sigma}\right)\right\rangle_{C_{g, n}}$ (4.4) in the limit $b=1$ exactly reproduces Pestun's formula (1.3) for the expectation value of a Wilson loop in the corresponding $\mathcal{N}=2$ theory.

Gauge theory operators carrying magnetic charge in a duality frame $\sigma$ are captured by the monodromy acquired by the conformal blocks when a degenerate field is transported around a curve $\gamma$, homotopic to a path in $\Gamma_{\sigma}$. This implies that the quantum numbers of the edges traversed by the degenerate field are shifted due to their OPE with the degenerate field which is being transported. Therefore, the conformal blocks are not eigenstates of Liouville loop operators exploring the trivalent graph. Nevertheless, their action on the conformal blocks is rather simple, and involves elementary shifts of the quantum numbers labeling the conformal block. These shifted conformal blocks capture 't Hooft and dyonic operators in the gauge theory.

We also note that our proposal, together with the modular invariance of the Liouville loop operator correlators, implies the conjectured action of the S-duality duality group $\Gamma\left(\mathcal{T}_{g, n}\right)$ on gauge theory loop operators, which states that

$$
\left\langle L_{d, \sigma}\right\rangle_{\mathcal{T}_{g, n}}=\left\langle L_{d^{\prime}, \sigma^{\prime}}\right\rangle_{\mathcal{T}_{g, n}}
$$

where $d$ and $d^{\prime}$ are the charges of the loop operator in the $\mathcal{T}_{g, n}$ duality frames $\sigma$ and $\sigma^{\prime}$ respectively.

\section{Computation of gauge theory loop operators}

In this section we explicitly use the identification of loop operators in Liouville theory on $C_{g, n}$ with loop operators in $\mathcal{T}_{g, n}$ to calculate the exact expectation value of Wilson loop operators in $\mathcal{T}_{g, n}$ and the expectation value of 't Hooft operators in two interesting examples: the $\mathcal{N}=2 \mathrm{SU}(2)$ theory with $N_{F}=4$ and the $\mathcal{N}=2^{*}$ theory. We then proceed to define operators on a pair of pants, which by composing them, computes the expectation value of complicated loop operator in any of the $\mathcal{T}_{g, n}$ theories.

\subsection{Preliminaries}

As explained in the previous section, the calculation of the monodromy of a degenerate field as it moves around a closed curve in $C_{g, n}$ can be broken into a set of moves on the trivalent graphs on which the conformal blocks are defined. It is a non-trivial fact of two dimensional conformal field theory that any allowed move can be obtained by composing the basic moves: fusion, braiding and S-move. In this subsection we introduce the key ingredients and formulas behind the basic moves we need. 
The moves are local on the trivalent graph. They involve at most five consecutive edges. On such a graph we can define the $s$-channel and $t$-channel 4-pt BPZ conformal blocks (2.5)

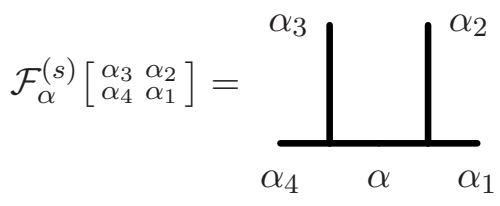

$$
\begin{aligned}
& \mathcal{F}_{\alpha}^{(t)}\left[\begin{array}{ll}
\alpha_{3} & \alpha_{2} \\
\alpha_{4} & \alpha_{1}
\end{array}\right]=\underbrace{\alpha_{3}}_{\alpha_{4}} Y_{1}^{\alpha_{2}}
\end{aligned}
$$

In the explicit calculations in this section we employ for calculational convenience a different set of conformal blocks $\mathcal{G}_{\alpha, E}^{(\sigma)}$ which absorb most of the Liouville three-point function $[15,33,34]$ into the blocks themselves. ${ }^{23}$

In terms of these conformal blocks, the Liouville correlation function (2.6) is written as

$$
\left\langle\prod_{a=1}^{n} V_{m_{a}}\right\rangle_{C_{g, n}}=\int d \mu(\alpha) \overline{\mathcal{G}}_{\alpha, E}^{(\sigma)} \mathcal{G}_{\alpha, E}^{(\sigma)}
$$

where the measure is

$$
d \mu(\alpha)=\prod_{i=1}^{3 g-3+n} d \alpha_{i}\left(4 \sin \left(\pi b\left(2 \alpha_{i}-Q\right)\right) \sin \left(\frac{\pi}{b}\left(Q-2 \alpha_{i}\right)\right)\right)
$$

The precise relation between the two involves multiplying the $\mathcal{F}$ blocks by one factor of $N\left(\alpha_{3}, \alpha_{2}, \alpha_{1}\right)$ for each vertex in the graphs $\Gamma_{\sigma}$ where [42]

$$
N\left(\alpha_{1}, \alpha_{2}, \alpha_{3}\right)=\frac{\Gamma_{b}\left(2 Q-\alpha_{1}-\alpha_{2}-\alpha_{3}\right) \Gamma_{b}\left(\alpha_{1}+\alpha_{2}-\alpha_{3}\right) \Gamma_{b}\left(\alpha_{1}+\alpha_{3}-\alpha_{2}\right) \Gamma_{b}\left(\alpha_{2}+\alpha_{3}-\alpha_{1}\right)}{\Gamma_{b}\left(2 Q-2 \alpha_{1}\right) \Gamma_{b}\left(2 \alpha_{2}\right) \Gamma_{b}\left(2 \alpha_{3}\right)},
$$

and $\Gamma_{b}(x)$ is the Barnes double Gamma function (see appendix A for the definition and a collection of properties of this function and the closely related $S_{b}(x)$ and $\Upsilon(s)$ ). The function $N\left(\alpha_{1}, \alpha_{2}, \alpha_{3}\right)$ is related to the Liouville three-point function $C\left(\alpha_{1}, \alpha_{2}, \alpha_{3}\right)[33,34]$ through [42]

$$
C\left(\alpha_{1}, \alpha_{2}, \alpha_{3}\right)=\left(\pi \mu \frac{\Gamma\left(b^{2}\right)}{\Gamma\left(1-b^{2}\right)} b^{2-2 b^{2}}\right)^{\frac{1}{b}\left(Q-\alpha_{1}-\alpha_{2}-\alpha_{3}\right)} \Upsilon^{\prime}(0)\left|N\left(\alpha_{1}, \alpha_{2}, \alpha_{3}\right)\right|^{2} \prod_{i=1}^{3} \frac{\Gamma_{b}\left(2 Q-2 \alpha_{i}\right)}{\Gamma_{b}\left(Q-2 \alpha_{i}\right)}
$$

In the product of all the three-point functions for the different vertices, the only terms which depend on the internal labels $\alpha$ are the $N\left(\alpha_{1}, \alpha_{2}, \alpha_{3}\right)$ factors and some of the ratios of Barnes functions, which give the measure (5.4). All the rest of the terms can be absorbed in the normalizations of the external legs. Note also that for normalizable states, when $\bar{\alpha}_{1}=Q-\alpha_{1}$, then $\left|N\left(\alpha_{1}, \alpha_{2}, \alpha_{3}\right)\right|^{2}=\left|N\left(Q-\alpha_{1}, \alpha_{2}, \alpha_{3}\right)\right|^{2}$.

\footnotetext{
${ }^{23}$ These are also slightly different from the conventions of [13].
} 
In the case of the four-point s-channel and t-channel conformal blocks above the explicit mapping is

$$
\begin{aligned}
& \mathcal{G}_{\alpha}^{(s)}\left[\begin{array}{cc}
\alpha_{3} & \alpha_{2} \\
\alpha_{4} & \alpha_{1}
\end{array}\right]=N\left(\alpha_{4}, \alpha_{3}, \alpha\right) N\left(\alpha, \alpha_{2}, \alpha_{1}\right) \mathcal{F}_{\alpha}^{(s)}\left[\begin{array}{cc}
\alpha_{3} & \alpha_{2} \\
\alpha_{4} & \alpha_{1}
\end{array}\right] \\
& \mathcal{G}_{\alpha}^{(t)}\left[\begin{array}{ll}
\alpha_{3} & \alpha_{2} \\
\alpha_{4} & \alpha_{1}
\end{array}\right]=N\left(\alpha_{4}, \alpha, \alpha_{1}\right) N\left(\alpha, \alpha_{3}, \alpha_{2}\right) \mathcal{F}_{\alpha}^{(t)}\left[\begin{array}{ll}
\alpha_{3} & \alpha_{2} \\
\alpha_{4} & \alpha_{1}
\end{array}\right],
\end{aligned}
$$

A fusion move is a transformation that relates the $s$-channel conformal blocks to the $t$-channel conformal blocks. They are related by a change of basis. The moves on the BPZ conformal blocks for Liouville theory are

$$
\underset{\alpha_{4} \quad \alpha \alpha_{1}}{\left.\alpha_{3}^{\alpha_{3}}\right|_{\alpha_{4}} ^{\alpha_{2}} \alpha_{1}} \underbrace{\alpha_{3}}_{\alpha^{\prime}}:=\mathcal{F}_{\alpha}^{(s)}\left[\begin{array}{cc}
\alpha_{3} & \alpha_{2} \\
\alpha_{4} & \alpha_{1}
\end{array}\right]=\int d \alpha^{\prime} F_{\alpha \alpha^{\prime}}\left[\begin{array}{ll}
\alpha_{3} & \alpha_{2} \\
\alpha_{4} & \alpha_{1}
\end{array}\right] \mathcal{F}_{\alpha^{\prime}}^{(t)}\left[\begin{array}{ll}
\alpha_{3} & \alpha_{2} \\
\alpha_{4} & \alpha_{1}
\end{array}\right]
$$

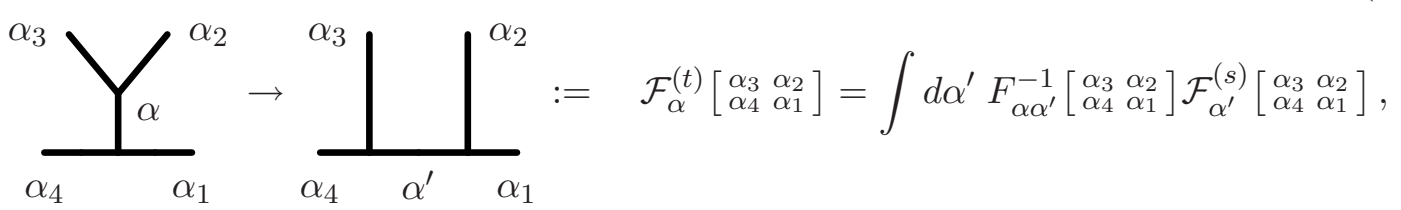

where $F_{\alpha \alpha^{\prime}}\left[\begin{array}{cc}\alpha_{1} & \alpha_{2} \\ \alpha_{4} & \alpha_{3}\end{array}\right]$ are called the fusion matrices. The forward and inverse transformation are simply related by

$$
F_{\alpha \alpha^{\prime}}^{-1}\left[\begin{array}{ll}
\alpha_{3} & \alpha_{2} \\
\alpha_{4} & \alpha_{1}
\end{array}\right]=F_{\alpha \alpha^{\prime}}\left[\begin{array}{ll}
\alpha_{1} & \alpha_{2} \\
\alpha_{4} & \alpha_{3}
\end{array}\right] .
$$

The fusion matrices for the $\mathcal{G}$ blocks (5.7) are therefore

$$
G_{\alpha \alpha^{\prime}}\left[\begin{array}{cc}
\alpha_{3} & \alpha_{2} \\
\alpha_{4} & \alpha_{1}
\end{array}\right]=\frac{N\left(\alpha_{4}, \alpha_{3}, \alpha\right) N\left(\alpha, \alpha_{2}, \alpha_{1}\right)}{N\left(\alpha_{4}, \alpha^{\prime}, \alpha_{1}\right) N\left(\alpha^{\prime}, \alpha_{3}, \alpha_{2}\right)} F_{\alpha \alpha^{\prime}}\left[\begin{array}{cc}
\alpha_{3} & \alpha_{2} \\
\alpha_{4} & \alpha_{1}
\end{array}\right]
$$

and satisfy the same symmetry properties as the fusion matrices for the $\mathcal{F}$ blocks

$$
G_{\alpha \alpha^{\prime}}\left[\begin{array}{ll}
\alpha_{3} & \alpha_{2} \\
\alpha_{4} & \alpha_{1}
\end{array}\right]=G_{\alpha \alpha^{\prime}}\left[\begin{array}{lll}
\alpha_{2} & \alpha_{3} \\
\alpha_{1} & \alpha_{4}
\end{array}\right]=G_{\alpha \alpha^{\prime}}\left[\begin{array}{lll}
\alpha_{4} & \alpha_{1} \\
\alpha_{3} & \alpha_{2}
\end{array}\right]=G_{\alpha \alpha^{\prime}}\left[\begin{array}{lll}
\alpha_{1} & \alpha_{4} \\
\alpha_{2} & \alpha_{3}
\end{array}\right]
$$

The other operation we will employ is the braiding move. This move exchanges two consecutive edges in a planar graph. In what follows, we will usually perform braiding twice, so we return to a diagram which looks like the original one, only that we do have to remember that a "Dehn-twist" was performed along a tube on the Riemann surface. Pictorically, the braiding move is given by

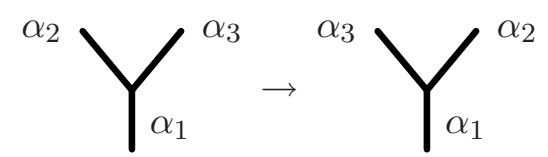

Braiding introduces the following phase into the conformal blocks

$$
B_{\alpha_{1}}^{\alpha_{2} \alpha_{3}}=e^{i \pi\left(\Delta\left(\alpha_{1}\right)-\Delta\left(\alpha_{2}\right)-\Delta\left(\alpha_{3}\right)\right)},
$$

where $\Delta(\alpha)=\alpha(Q-\alpha)$ is the conformal dimension of the operator $V_{\alpha}$. Equipped with the action of fusion and braiding on the conformal blocks we now proceed to calculate the expectation value of Liouville loop operators. 


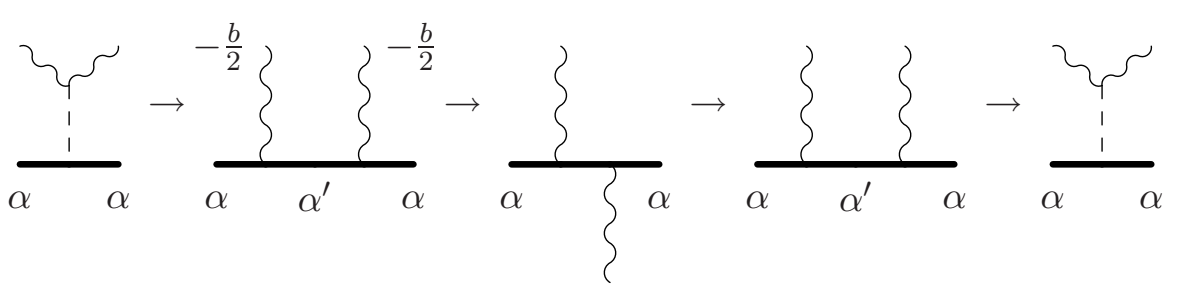

Figure 2. Calculating the Wilson loop in the fundamental representation. The solid lines carry arbitrary states, the wiggly ones are degenerate fields and the dashed line carries the identity state.

\subsection{Wilson loops from Liouville theory}

In [25] the Wilson loop operators in $\mathcal{T}_{g, n}$ were identified with closed curves that wind around any of the $3 g-3+n$ cycles along which the surface $C_{g, n}$ is sewn from pairs of pants. Let us label a curve along one such cycle by $\gamma_{0,1}$.

We first start by considering the simplest Wilson loop, which transforms in the $j=1 / 2$ representation with respect to the $\mathrm{SU}(2)$ gauge group corresponding to the tube that the curve $\gamma_{0,1}$ encircles. The relevant gauge group is identified with an internal edge in the generalized quiver $\Gamma_{\sigma}$.

We need to calculate the monodromy of a $V_{1,2}$ degenerate field as it moves around the curve $\gamma_{0,1}$. As the operation is local on $\Gamma_{\sigma}$, we ignore the rest of the trivalent graph and suppress all other indices in the conformal blocks, except for the quantum number labeling the internal edge in the generalized quiver $\Gamma_{\sigma}$ that corresponds to the desired $\mathrm{SU}(2)$ gauge group, which we label by the quantum number $\alpha$.

In order to probe the edge labeled by $\alpha$ we need to introduce a degenerate operator $V_{1,2}$ and braid it around. Introducing a single operator will necessarily change the edge in a way that if it carries on one side an intermediate state $\alpha$, then on the other it will carry $\alpha \pm b / 2$, which follows from the OPE of the $V_{1,2}$ degenerate field (4.1). To avoid this, as explained in section 4 , we introduce a pair of $V_{1,2}$ degenerate operators with their OPE projected to the identity. This configuration is represented by the left-most diagram in figure 2 .

By a fusion move we can then express the same configuration in the $s$-channel, where we sum over the intermediate states $\alpha^{\prime}=\alpha-s b / 2$ between the pair of degenerate fields, where $s= \pm 1$. Now we perform a non-trivial operation of moving one of the degenerate fields around, corresponding to a braiding move, fusing it back together with the other degenerate field and projecting onto the identity.

Given this sequence of moves, we can calculate the action of the loop operator $\mathcal{L}\left(\gamma_{0,1}\right)$ on the conformal block by using the explicit form of the fusion matrices (see appendix $\mathrm{B}$ for the explicit expression of all the fusion matrices required in our calculations). Since the only relevant label in the conformal block is the one associated to the quantum number $\alpha$, we do not write explicitly all the other quantum numbers in the conformal block. The action of the loop operator $\mathcal{L}\left(\gamma_{0,1}\right)$ on the conformal block is given by the following sequence 
of fusion and braiding steps

$$
\begin{aligned}
{\left[\mathcal{L}\left(\gamma_{0,1}\right) \cdot \mathcal{G}\right]_{\alpha} } & =\sum_{s= \pm} G_{0, \alpha^{\prime}}^{-1}\left[\begin{array}{cc}
-\frac{b}{2} & -\frac{b}{2} \\
\alpha & \alpha
\end{array}\right]\left(B_{\alpha^{\prime}}^{-\frac{b}{2}, \alpha}\right)^{2} G_{\alpha^{\prime}, 0}\left[\begin{array}{cc}
-\frac{b}{2} & -\frac{b}{2} \\
\alpha^{2}
\end{array}\right] \mathcal{G}_{\alpha} \\
& =\sum_{s= \pm} G_{-, s}\left[\begin{array}{c}
\alpha-\frac{b}{2} \\
\alpha-\frac{b}{2}
\end{array}\right] e^{\pi b i(Q+s(2 \alpha-Q))} G_{s,-}\left[\begin{array}{cc}
-\frac{b}{2}-\frac{b}{2} \\
\alpha^{2}
\end{array}\right] \mathcal{G}_{\alpha} \\
= & \sum_{s= \pm} \frac{\sin \left(\pi b^{2}\right)}{\sin (\pi b(2 \alpha-Q))} e^{\pi b i(Q+s(2 \alpha-Q))} \frac{\sin \left(\pi b\left(2 \alpha^{\prime}-Q\right)\right)}{\sin \left(2 \pi b^{2}\right)} \mathcal{G}_{\alpha}=\frac{\cos (\pi b(2 \alpha-Q))}{\cos (\pi b Q)} \mathcal{G}_{\alpha}
\end{aligned}
$$

Taking the $b \rightarrow 1$ limit, which corresponds to the partition function of $\mathcal{T}_{g, n}$ on $S^{4}$, gives

$$
\left[\mathcal{L}\left(\gamma_{0,1}\right) \cdot \mathcal{G}\right]_{\alpha}=\cos (2 \pi a) \mathcal{G}_{\alpha}, \quad \alpha=\frac{Q}{2}+a
$$

An interesting point about this formula is the normalization. It exactly matches the Wilson loop in the spin $1 / 2$ representation when the trace is normalized by dividing by the dimension of the representation, which is two for $j=1 / 2$. More generally, away from $b=1$, if we multiply the numerator by 2 then the denominator is $2 \cos (\pi b Q)=e^{i \pi b Q}+e^{-i \pi b Q}$, which is the quantum dimension of the spin $1 / 2$ representation of q-deformed $\operatorname{SL}(2, \mathbb{R})$.

The same expression would apply also to the conformal blocks with any of the other normalizations, since the operator $\mathcal{L}\left(\gamma_{0,1}\right)$ diagonalizes the conformal blocks, and acts by a multiplicative factor.

Using the expression for the correlator of a Liouville loop operator (4.4), we find that the result exactly matches Pestun's formula [10] for the expectation value of a Wilson loop in the $j=1 / 2$ representation for one of the gauge groups ${ }^{24}$

$$
\left\langle\mathcal{L}\left(\gamma_{0,1}\right)\right\rangle_{C_{g, n}}=\left\langle W_{j=1 / 2}\right\rangle_{\mathcal{T}_{g, n}}=\int[d a] \cos (2 \pi a) \bar{Z}_{\text {Nekrasov }} Z_{\text {Nekrasov }},
$$

where we have used the identification between the Liouville correlator and the partition function of the gauge theory without a loop operator found in [13].

We now proceed to calculate the expectation value of a Wilson loop in the spin $j=q / 2$ representation of one of the $\mathrm{SU}(2)$ groups. As discussed in section 3, the degenerate operator that must be moved around the Riemann surface is $V_{1, q+1}$. Since the product of two identical degenerate fields $V_{r, s}$ always contains the identity operator, we can carry out the same operation we did for the $V_{1,2}$ degenerate field. A pair of them can also be created in the same way as before, as discussed in detail in appendix B.1. The setup is precisely the same as the one in figure 2, but now the degenerate fields have quantum numbers $-q b / 2$. In this case, between the two degenerate fields $V_{1, q+1}$ the state $\alpha$ is shifted to $\alpha-k b / 2$ with $k=-q,-q+2, \ldots, q$, due to the OPE expansion of a $V_{1, q+1}$ degenerate field and a primary field. The action of the loop operator $\mathcal{L}\left(\gamma_{0,1}\right)$ on the conformal blocks

\footnotetext{
${ }^{24}$ We stress that the insertion of $\cos (2 \pi a)$ is for only one of the gauge fields.
} 
is therefore ${ }^{25}$

$$
\begin{aligned}
& {\left[\mathcal{L}_{q / 2}\left(\gamma_{0,1}\right) \cdot \mathcal{G}\right]_{\alpha}=\sum_{k} G_{0, \alpha-\frac{k b}{2}}^{-1}\left[\begin{array}{cc}
-\frac{q b}{2} & -\frac{q b}{2} \\
\alpha &
\end{array}\right]\left(B_{\alpha-k \frac{b}{2}}^{-q \frac{b}{2}, \alpha}\right)^{2} G_{\alpha-\frac{k b}{2}, 0}\left[\begin{array}{cc}
-\frac{q b}{2} & -\frac{q b}{2} \\
\alpha &
\end{array}\right] \mathcal{G}_{\alpha}} \\
& =\frac{\sum_{k} \exp [2 \pi i k b a]}{\sum_{k} \exp [\pi i k b Q]} \mathcal{G}_{\alpha} \text {. }
\end{aligned}
$$

As in the case of $j=1 / 2$, the denominator is the quantum dimension of the spin $q / 2$ representation of the q-deformed $\operatorname{SL}(2, \mathbb{R})$ (or the classical dimension for $b=1$ ).

In the limit $b \rightarrow 1$, where the Liouville correlator has an interpretation in term of $\mathcal{N}=2$ gauge theories on $S^{4}$ we get

$$
\left[\mathcal{L}_{q / 2}\left(\gamma_{0,1}\right) \cdot \mathcal{G}\right]_{\alpha}=\frac{1}{q+1} \sum_{k} \exp [2 \pi i k a] \mathcal{G}_{\alpha}
$$

This result exactly matches Pestun's formula [10] for the expectation value of the Wilson loop in the spin $j=q / 2$ representation for one of the gauge groups

$$
\left\langle\mathcal{L}_{q / 2}\left(\gamma_{0,1}\right)\right\rangle_{C_{g, n}}=\left\langle W_{q / 2}\right\rangle_{\mathcal{T}_{g, n}} .
$$

As discussed in section 3, there are two other natural bases of Wilson loop observables with quantum number $q$, corresponding to the multi-wrapped loops and the multi-trace loops. They also have a simple realization in terms of Liouville loop operators.

We can use the same degenerate field, and note that wrapping a single $V_{1,2}$ field $q$ times around the curve gives extra braiding factors in (5.15). The result is

$$
\begin{aligned}
{\left[\mathcal{L}\left(q \cdot \gamma_{0,1}\right) \cdot \mathcal{G}\right]_{\alpha} } & =\sum_{s= \pm} e^{q \pi b i(Q+s(2 \alpha-Q))} \frac{\sin \left(\pi b^{2}\right)}{\sin \left(2 \pi b^{2}\right)} \frac{\sin \left(\pi b\left(2 \alpha^{\prime}-Q\right)\right)}{\sin (\pi b(2 \alpha-Q))} \mathcal{G}_{\alpha} \\
& \longrightarrow \cos (2 \pi q a) \mathcal{G}_{\alpha} .
\end{aligned}
$$

This yields the expectation value of the multi-wrapped Wilson loop in the $\mathcal{N}=2$ gauge theory ${ }^{26}$

$$
\left\langle\mathcal{L}\left(q \cdot \gamma_{0,1}\right)\right\rangle_{C_{g, n}}=\left\langle\frac{1}{2} \operatorname{Tr} e^{2 \pi i q a}\right\rangle_{\mathcal{T}_{g, n}} .
$$

Instead, if we consider $q$ pairs of independent degenerate fields we just get the $q^{\text {th }}$ power of the previous result

$$
\left[\mathcal{L}\left(\gamma_{0,1}\right)^{q} \cdot \mathcal{G}\right]_{\alpha}=\left[-\frac{\cos (\pi b(2 \alpha-Q))}{\cos \left(\pi b^{2}\right)}\right]^{q} \mathcal{G}_{\alpha} \quad \underset{b \rightarrow 1}{\longrightarrow} \quad \cos ^{q}(2 \pi a) \mathcal{G}_{\alpha}
$$

This yields the expectation value of the multi-trace Wilson loop in the $\mathcal{N}=2$ gauge theory

$$
\left\langle\mathcal{L}\left(\gamma_{0,1}\right)^{q}\right\rangle_{C_{g, n}}=\left\langle\left[W_{1 / 2}\right]^{q}\right\rangle_{\mathcal{T}_{g, n}}
$$

We thus have found a Liouville description for each of the three natural Wilson loops with quantum number $q$ :

\footnotetext{
${ }^{25}$ This expression was checked up to $q=5$, see also equation (B.18).

${ }^{26}$ On the right-hand side we are using notations from Pestun's matrix model calculation to represent the corresponding field theory observable.
} 


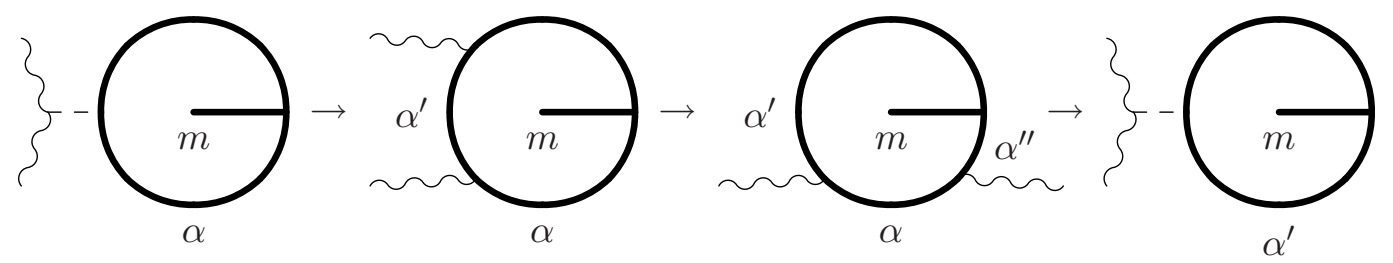

Figure 3. Calculating the 't Hooft loop on the one-punctured torus.

1. The Wilson loop in the irreducible $j=q / 2$ representation is given by generating a pair of $V_{1, q+1}$ degenerate fields, braiding one of them around and fusing them back into the identity state.

2. The Wilson loop wrapped $q$ times around the curve is given by generating a pair of the basic $V_{1,2}$ degenerate fields, braiding one of them around $q$ times and fusing them back into the identity state. This can be thought of as a $q$-times wound curve.

3. The $q^{\text {th }}$ product of the fundamental Wilson loop is achieved by generating $q$ pairs of the basic $V_{1,2}$ degenerate fields, braiding one of each pair and fusing them back into the identity states. This can be thought of as $q$ parallel curves on the surface.

Combinations of any of these can be used to create all other Wilson loop operators.

\section{3 't Hooft loop in $\mathcal{N}=2^{*}$ theory}

As discussed earlier, from the Liouville point of view 't Hooft loop operators are also described by the monodromy of the conformal block associated to a degenerate field moving around a closed curve. Although these are essentially the same calculations we performed for the Wilson loop, once we choose a different pants-decomposition of the Riemann surface the details change drastically.

Given a choice of pants decomposition $\sigma$ of the Riemann surface, the curve that describes a loop operator carrying magnetic charges must move nontrivially around the corresponding trivalent graph $\Gamma_{\sigma}$. For the case of the Wilson loop just considered, the associated curve simply corresponds to a rotation around one the edges of $\Gamma_{\sigma}$. Since the degenerate field does not move in $\Gamma_{\sigma}$, one would expect that the loop operator does not change the quantum number propagating along the edge, which is what we have found. For an operator carrying magnetic charge the curve must explore the trivalent graph $\Gamma_{\sigma}$, which suggests that the quantum numbers associated to the edges traversed by the curve are now affected by the action of the loop operator. As we show, this intuitive picture is materialized in the actual computation of the monodromy.

We now consider an 't Hooft operator in $\mathcal{N}=2^{*}$, which corresponds to the oncepunctured torus. As usual we create a pair of degenerate fields which project to the identity operator (see the first picture in figure 3). If in the second step, after fusion, we move one of the degenerate fields around the graph without changing the location of the 
degenerate field on the graph, then this corresponds to the Wilson loop calculation we have already carried out.

Transporting the degenerate field around the entire graph corresponds instead to the 't Hooft operator. The procedure is illustrated in figure 3. A pair of degenerate fields, which combine to the identity are rewritten in the dual channel with an intermediate state $\alpha^{\prime}=\alpha-s b / 2$, where $s= \pm 1$. Then one of the degenerate fields is fused across the puncture (with label $m$ ). In principle one should allow for a new state $\alpha^{\prime \prime}$ there, but since we then combine again the two degenerate fields and project onto the identity, this identifies the two quantum numbers $\alpha^{\prime \prime}=\alpha^{\prime}$.

There is a subtle point about this calculation, which is the exact identification of the initial and final state. Great care has to be taken to guarantee that we follow exactly the same path as in the Wilson loop calculation (in the dual channel), and do not permute the two degenerate fields. Otherwise one ends up with extra phases (5.14) due to braiding between these two fields. Note that the ambiguity is independent of $\alpha$ and $m$, so we can examine the case of $m=0$ (torus without a puncture), where the operator $M_{\sigma^{\prime} \sigma}$ in (4.7) is just Fourier-transformation. In that case the transformation of the difference operator $\mathcal{D}_{\alpha}^{(\sigma)}$ is obvious, and gives the formula below. This fixes the possible phase. ${ }^{27}$

This sequence of moves induces a monodromy on the conformal block of the once punctured torus, which we denote by $\mathcal{G}_{\alpha, m}$. The fusion and braiding moves yield

$$
\begin{gathered}
{\left[\mathcal{L}\left(\gamma_{1,0}\right) \cdot \mathcal{G}\right]_{\alpha, m}=\sum_{s= \pm} G_{0, \alpha-\frac{s b}{2}}\left[\begin{array}{l}
\alpha-\frac{b}{2} \\
\alpha-\frac{b}{2}
\end{array}\right] G_{\alpha, \alpha-\frac{s b}{2}}^{-1}\left[\begin{array}{cc}
\alpha-s \frac{b}{2}-\frac{b}{2} \\
m
\end{array}\right] G_{\alpha, 0}\left[\begin{array}{cc}
\alpha-s \frac{b}{2} \alpha-s \frac{b}{2} \\
-\frac{b}{2} & -\frac{b}{2}
\end{array}\right] \mathcal{G}_{\alpha-s b / 2, m}} \\
\quad=\sum_{s= \pm} G_{-, s}\left[\begin{array}{l}
\alpha-\frac{b}{2} \\
\alpha-\frac{b}{2}
\end{array}\right] G_{-s, s}\left[\begin{array}{cc}
\alpha & -\frac{b}{2} \\
m & \alpha-s \frac{b}{2}
\end{array}\right] G_{-s,-}\left[\begin{array}{cc}
-\frac{b}{2} & -\frac{b}{2} \\
\alpha-s \frac{b}{2} \alpha-s \frac{b}{2}
\end{array}\right] \mathcal{G}_{\alpha-s b / 2, m} \\
=\frac{\sin \left(\pi b^{2}\right)}{\sin \left(2 \pi b^{2}\right)}\left(\frac{\sin (\pi b(2 \alpha-Q-m))}{\sin (\pi b(2 \alpha-Q))} \mathcal{G}_{\alpha-b / 2, m}+\frac{\sin (\pi b(2 \alpha-Q+m))}{\sin (\pi b(2 \alpha-Q))} \mathcal{G}_{\alpha+b / 2, m}\right)
\end{gathered}
$$

We note that, as expected, the loop operator shifts the value of the quantum number associated with the edge along which the degenerate field is transported. Hence, unlike the calculation of the Wilson loop, the functional prefactors are then dependent on the normalization conventions for the conformal blocks, whether $\mathcal{G}, \mathcal{F}$ or $Z_{\text {Nekrasov }}{ }^{28}$

In the limit $b \rightarrow 1$, where the Liouville correlator has an interpretation in term of $\mathcal{N}=2^{*}$ on $S^{4}$, this yields

$$
\left\langle\mathcal{L}\left(\gamma_{1,0}\right)\right\rangle_{C_{g, n}}=2 \int d \alpha \sin (2 \pi \alpha) \overline{\mathcal{G}}_{\alpha, m}\left[\sin (\pi(2 \alpha-m)) \mathcal{G}_{\alpha-1 / 2, m}+\sin (\pi(2 \alpha+m)) \mathcal{G}_{\alpha+1 / 2, m}\right] .
$$

Our proposal (1.4) identifies the result of this calculation as the expectation value of an 't Hooft operator $T \equiv L_{1,0}$ in the $\mathcal{N}=2^{*}$ theory

$$
\langle T\rangle_{\mathcal{T}_{g, n}}=\left\langle\mathcal{L}\left(\gamma_{1,0}\right)\right\rangle_{C_{g, n}}
$$

\footnotetext{
${ }^{27}$ Also, since we know that the loop operators are Hermitian and positive definite (see section 6 ) this, in principle fixes the phase.

${ }^{28}$ To be explicit, for the one-punctured torus $\mathcal{G}_{\alpha, m}=N(\alpha, m, \alpha) \mathcal{F}_{\alpha, m}(5.5)$.
} 


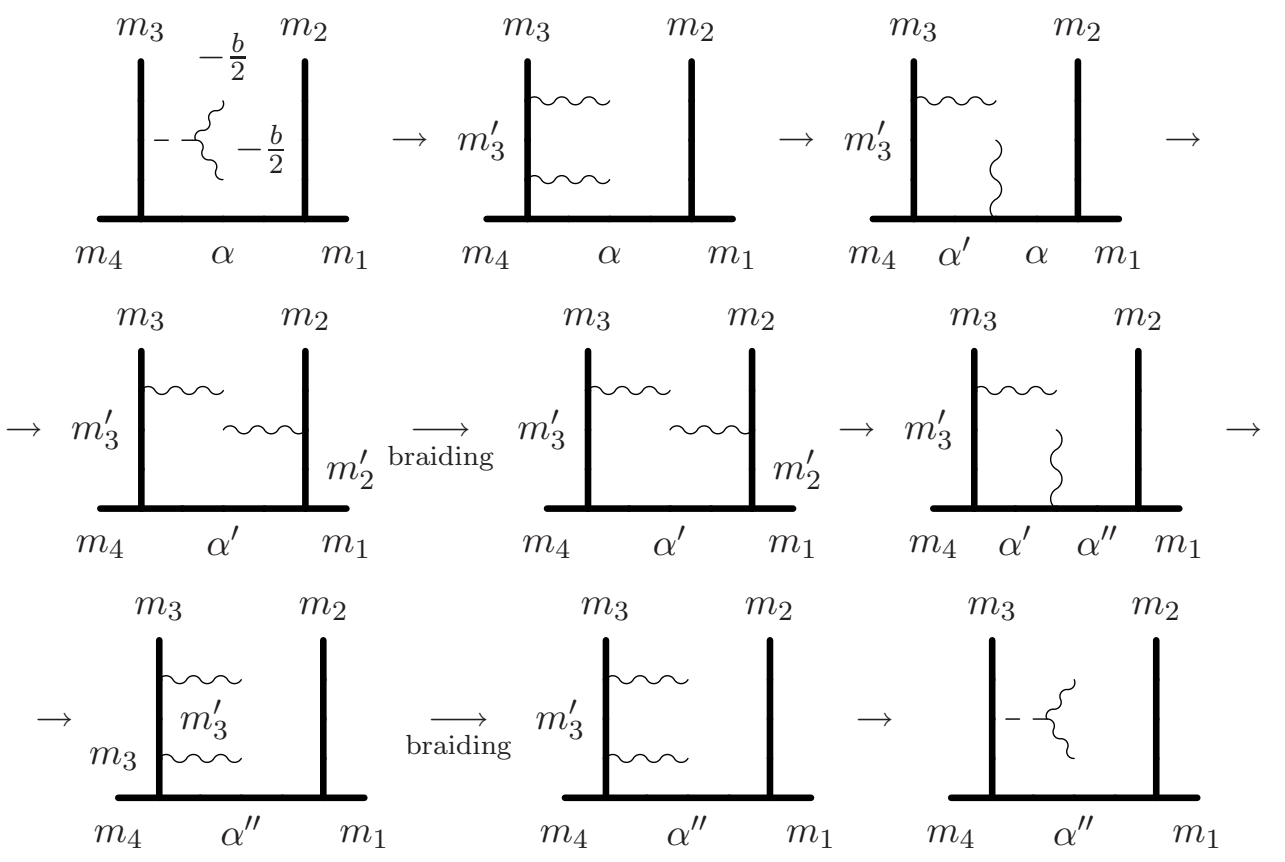

Figure 4. Calculating the 't Hooft loop on the four-punctured sphere as the monodromy associated to moving a $V_{1,2}$ field.

The 't Hooft loop is given by a sum of two terms, one where the argument of the holomorphic contribution is shifted by $1 / 2$ and the other by $-1 / 2$. In section 8 , we provide a possible gauge theory interpretation of this observation.

It is straightforward to generalize the calculation to the dyonic loop operator $\mathcal{L}\left(\gamma_{1, q}\right)$ by including an extra braiding factor. The result is

$$
\begin{aligned}
{\left[\mathcal{L}\left(\gamma_{1, q}\right) \cdot \mathcal{G}\right]_{\alpha, m}=e^{-q \pi i b^{2} / 2} \frac{\sin \left(\pi b^{2}\right)}{\sin \left(2 \pi b^{2}\right)}(} & e^{\pi i q b(2 \alpha-Q)} \frac{\sin (\pi b(2 \alpha-Q-m))}{\sin (\pi b(2 \alpha-Q))} \mathcal{G}_{\alpha-b / 2, m} \\
& \left.+e^{-\pi i q b(2 \alpha-Q)} \frac{\sin (\pi b(2 \alpha-Q+m))}{\sin (\pi b(2 \alpha-Q))} \mathcal{G}_{\alpha+b / 2, m}\right) .
\end{aligned}
$$

In section 7 we provide some consistency checks for the validity of this calculation by demonstrating that the dyonic operators satisfy the expected 't Hooft commutation relations for $b=1$ and the expected operator product expansion for $b=0$.

\section{4 't Hooft loop in the theory with one $\mathrm{SU}(2)_{C}$ and $N_{F}=4$}

The next theory we consider is $\mathcal{T}_{0,4}$, which is based on the four punctured sphere. This theory admits a weakly coupled description in terms of an $\mathcal{N}=2 \mathrm{SU}(2)$ gauge theory with four fundamental hypermultiplets. Our goal is to compute the expectation value of an 't Hooft loop in this theory.

The monodromy corresponding to the 't Hooft loop is captured by the series of moves illustrated in figure 4. First we insert a pair of degenerate fields in the edge labeled by $m_{3}$. 
One of the degenerate fields is then moved across the trivalent graph and then transported back. Note that when the degenerate field hits the vertex with external edges labeled by $m_{1}$ and $m_{2}$ braiding around the punctures must be performed. Likewise, we must braid when the degenerate field is back into the original vertex labeled by $m_{3}$ and $m_{4}$.

Using the formulae for the fusion and braiding moves we find that the expression for the complete move is the following

$$
\begin{aligned}
T_{s, s^{\prime}}=\sum_{s_{2}, s_{3}= \pm} G_{0 m_{3}^{\prime}}\left[\begin{array}{cc}
m_{3} & -\frac{b}{2} \\
m_{3} & -\frac{b}{2}
\end{array}\right] G_{m_{3} \alpha^{\prime}}^{-1}\left[\begin{array}{cc}
m_{3}^{\prime} & -\frac{b}{2} \\
m_{4} & \alpha
\end{array}\right] G_{\alpha, m_{2}^{\prime}}\left[\begin{array}{cc}
-\frac{b}{2} & m_{2} \\
\alpha^{\prime} & m_{1}
\end{array}\right]\left(B_{m_{2}^{\prime}}^{-\frac{b}{2}, m_{2}}\right)^{2} \\
\times G_{m_{2}^{\prime} \alpha^{\prime \prime}}^{-1}\left[\begin{array}{cc}
-\frac{b}{2} & m_{2} \\
\alpha^{\prime} & m_{1}
\end{array}\right] G_{\alpha^{\prime} m_{3}}\left[\begin{array}{cc}
m_{3}^{\prime} & -\frac{b}{2} \\
m_{4} & \alpha^{\prime \prime}
\end{array}\right]\left(B_{m_{3}^{\prime}}^{-\frac{b}{2}, m_{3}}\right)^{-2} G_{m_{3}^{\prime} 0}^{-1}\left[\begin{array}{cc}
m_{3}-\frac{b}{2} \\
m_{3} & -\frac{b}{2}
\end{array}\right],
\end{aligned}
$$

where

$$
\alpha^{\prime}=\alpha-\frac{s b}{2}, \quad \alpha^{\prime \prime}=\alpha-\frac{\left(s+s^{\prime}\right) b}{2}, \quad m_{2}^{\prime}=m_{2}-\frac{s_{2} b}{2}, \quad m_{3}^{\prime}=m_{3}-\frac{s_{3} b}{2},
$$

and $s, s^{\prime}, s_{2}$ and $s_{3}$ are either 1 or -1 .

The loop operator corresponding to the curve traced by this move - denoted by $\mathcal{L}\left(\gamma_{2,0}\right)$ - acts on the conformal blocks on the four punctured sphere, which we label by $\mathcal{G}_{\alpha}$ (we omit the labels $m_{1}, \ldots, m_{4}$ to avoid unnecessary clutter). The resulting monodromy is represented by the expression (5.29) multiplying the conformal blocks, where the intermediate state $\alpha$ is replaced by $\alpha^{\prime \prime}=\alpha-k b / 2$. We get the sum of three terms for $k=2,0,-2$ where compared to (5.29) we use $T_{2}=T_{++}, T_{0}=T_{+-}+T_{-+}$and $T_{-2}=T_{--}$

$$
\left[\mathcal{L}\left(\gamma_{2,0}\right) \cdot \mathcal{G}\right]_{\alpha}=T_{2} \mathcal{G}_{\alpha-b}+T_{0} \mathcal{G}_{\alpha}+T_{-2} \mathcal{G}_{\alpha+b}
$$

Explicitly the prefactors are

$$
\begin{aligned}
T_{2}= & -4 \frac{\sin \left(\pi b^{2}\right) \sin \left(\pi b\left(\alpha+m_{2}-m_{1}-b\right)\right) \sin \left(\pi b\left(\alpha-m_{2}+m_{1}-b\right)\right)}{\sin \left(2 \pi b^{2}\right) \sin (2 \pi b(\alpha-b)) \sin (\pi b(2 \alpha-b))} \\
& \times \sin \left(\pi b\left(\alpha+m_{3}-m_{4}-b\right)\right) \sin \left(\pi b\left(\alpha-m_{3}+m_{4}-b\right)\right) \\
T_{0}= & \frac{\left[\cos (\pi b(2 \alpha-b)) \cos \left(\pi b\left(2 m_{2}-b\right)\right)-\cos \left(\pi b^{2}\right) \cos \left(\pi b\left(2 m_{1}-b\right)\right)\right]}{\cos ^{2}\left(\pi b^{2}\right) \sin (2 \pi b(\alpha-b)) \sin (2 \pi b \alpha)} \\
& \times\left[\cos (\pi b(2 \alpha-b)) \cos \left(\pi b\left(2 m_{3}-b\right)\right)-\cos \left(\pi b^{2}\right) \cos \left(\pi b\left(2 m_{4}-b\right)\right)\right] \\
& +\frac{\cos \left(\pi b\left(2 m_{2}-b\right)\right) \cos \left(\pi b\left(2 m_{3}-b\right)\right)}{\cos ^{2}\left(\pi b^{2}\right)} \\
T_{-2}= & -4 \frac{\sin \left(\pi b^{2}\right) \sin \left(\pi b\left(\alpha+m_{2}+m_{1}-b\right)\right) \sin \left(\pi b\left(\alpha-m_{2}-m_{1}-b\right)\right)}{\sin \left(2 \pi b^{2}\right) \sin (2 \pi b \alpha) \sin (\pi b(2 \alpha-b))} \\
& \times \sin \left(\pi b\left(\alpha+m_{3}+m_{4}-b\right)\right) \sin \left(\pi b\left(\alpha-m_{3}-m_{4}-b\right)\right)
\end{aligned}
$$

In section 7 we provide some consistency checks for the validity of this calculation by demonstrating that the Wilson and 't Hooft loop operators satisfy the expected 't Hooft commutation relation for $b=1$ and the expected operator product expansion for $b=0$. 
In the limit $b \rightarrow 1$, where the Liouville correlator has an interpretation in term of the $\mathcal{N}=2 \mathrm{SU}(2)$ gauge theory with $N_{F}=4$ on $S^{4}$ we get

$$
\left\langle\mathcal{L}\left(\gamma_{2,0}\right)\right\rangle_{C_{g, n}}=\int d \alpha \sin ^{2}(2 \pi \alpha)\left[\overline{\mathcal{G}}_{\alpha} T_{2}^{\prime} \mathcal{G}_{\alpha-1}+\overline{\mathcal{G}}_{\alpha} T_{0}^{\prime} \mathcal{G}_{\alpha}+\overline{\mathcal{G}}_{\alpha} T_{-2}^{\prime} \mathcal{G}_{\alpha+1}\right]
$$

where

$$
\begin{aligned}
T_{2}^{\prime}= & \frac{2}{\sin ^{2}(2 \pi \alpha)} \sin \left(\pi\left(\alpha+m_{2}-m_{1}\right)\right) \sin \left(\pi\left(\alpha-m_{2}+m_{1}\right)\right) \\
& \times \sin \left(\pi\left(\alpha+m_{3}-m_{4}\right)\right) \sin \left(\pi\left(\alpha-m_{3}+m_{4}\right)\right) \\
T_{0}^{\prime}= & \frac{\left.\left.\left[\cos (2 \pi \alpha) \cos \left(2 \pi m_{2}\right)-\cos \left(2 \pi m_{1}\right)\right)\right]\left[\cos (2 \pi \alpha) \cos \left(2 \pi m_{3}\right)-\cos \left(2 \pi m_{4}\right)\right)\right]}{\sin ^{2}(2 \pi \alpha)} \\
& \left.+\cos \left(2 \pi m_{2}\right) \cos \left(2 \pi m_{3}\right)\right) \\
T_{-2}^{\prime}= & -\frac{2}{\sin ^{2}(2 \pi \alpha)} \sin \left(\pi\left(\alpha+m_{2}+m_{1}\right)\right) \sin \left(\pi\left(\alpha-m_{2}-m_{1}\right)\right) \\
& \times \sin \left(\pi\left(\alpha+m_{3}+m_{4}\right)\right) \sin \left(\pi\left(\alpha-m_{3}-m_{4}\right)\right)
\end{aligned}
$$

The ' $t$ Hooft loop is given by a sum of three terms, one where the argument of the holomorphic contribution is shifted by 1 , the other by -1 and one is unshifted. In section 8 , we provide a possible gauge theory interpretation of this observation.

\subsection{More general loop operators}

We have in previous subsections demonstrated the prescription of calculating the Liouville loop operator $\mathcal{L}(\gamma)$. This procedure can be applied to any collection of non-intersecting curves on the surface and by the conjectured relation between Liouville and the $\mathcal{N}=2$ gauge theories (as well as the explicit check on Wilson loops) calculate general Wilson, 't Hooft and dyonic loop operators in these gauge theories.

As reviewed in section 2, the classification of non-intersecting curves on the Riemann surface exactly matches the possible charges carried by loop operators in the gauge theory [25]. But we pointed out in section 3 and in section 5.2, there is more than one loop operator in Liouville theory associated to a given set of charges. The three cases we discussed are associated to the Wilson loop which is multi-wound, the multi-trace operator and the loop in a higher dimensional irreducible representation.

The distinction between the different loop operators is simply a change of basis, which can be seen both in the gauge theory and in Liouville theory. The multi-trace loops are in a product of the spin $1 / 2$ representation, which can be decomposed into irreducible representations. Similarly, the algebra of the degenerate fields is the quantum deformation of $\operatorname{sl}(2, \mathbb{R})$.

In this regard our analysis is complete, we found a Liouville operator for gauge theory operators with arbitrary charges, which should furnish a complete basis on both sides of the correspondence.

The main advantage of the operators built out of the monodromy of the $V_{1,2}$ degenerate field along non-intersecting curves is that this definition is modular invariant. The 
classification of loop operators matches Dehn's classification of the curves and the explicit transformation rules under a change of pants decomposition was presented by Penner [43]. Another technical simplification is the fact that the fusion rules for $V_{1,2}$ are the simplest.

There could be other bases which have other advantages and we present now a proposal for one. It has the advantage that it is local on the graph $\Gamma_{\sigma}$ for a fixed pants decomposition $\sigma$ which simplifies the calculation in that duality frame. This comes clearly at the price of not having simple modular properties. But such a description is likely to match specific gauge theory descriptions in the corresponding weakly coupled limit of $\mathcal{T}_{g, n}$.

We will consider loop operators made by splitting higher degenerate fields into collections of lower degenerate fields and fusing them back together along the Riemann surface. In particular given a pants decomposition $\sigma$, it decomposes the curve into arcs on the different pairs of pants, which is in fact a key step in Dehn's theorem. This operation groups different segments of the same curve as being parallel to each other on $\Gamma_{\sigma}$. It is then rather natural to replace a collection of parallel segments, with a single line for a higher degenerate field. ${ }^{29}$

Consider the case of the 't Hooft loop in the theory with $\mathcal{N}=2 \mathrm{SU}(2)$ gauge group with $N_{F}=4$ discussed in section 5.4. The loop is labeled by $\gamma_{2,0}$ and passes twice through the central edge in $\Gamma_{\sigma}$ (with the label $\alpha$ in figure 4 ). In fact one can consider a calculation where both of the degenerate fields are transported around the $m_{3}$, fused together, transported along the central edge, unfused into a pair of $V_{1,2}$ fields which then wrap the $m_{2}$ line.

Note that the fusion of the two degenerate fields will give the sum of the identity state and $V_{1,3}$. The contribution of the identity state is independent of $\alpha$, of $m_{1}$ and of $m_{4}$ and is the term on the last line of (5.33). The $V_{1,3}$ state gives all the other terms.

This decomposition of the loop operator into the contribution of the identity and $V_{1,3}$ along the intermediate edge should be mirrored by a decomposition of the analogous 't Hooft operator in the gauge theory into a triplet and a signlet. This decomposition is rather subtle and as we saw in the Liouville calculation requires the choice of a pair of punctures with labels $m_{2}$ and $m_{3}$ (similarly this can be done with the pair $m_{1}$ and $m_{4}$ ). As mentioned before, a fully detailed construction of loop operators in these gauge theories does not exist and the subtleties in the reduction to irreducible representations have not been addressed. We do find some indication for this decomposition in the algebra of loop operators in section 7 .

\subsubsection{Building loops from smaller constituents}

We would like to propose an algorithm that enables a relatively easy calculation of loop operators where all the arcs of the loop in a given pants decomposition are fused to higher degenerate fields (and roughly speaking should correspond to Wilson and 't Hooft loop operators in irreducible representations).

We recall the statement of Dehn's theorem, which analyzes curves on surfaces with specific pants decomposition. On each pair of pants there will be a collection of arcs going between the three boundaries. By a homotopy one can guarantee that all the arcs on all

\footnotetext{
${ }^{29} \mathrm{~A}$ similar idea, of allowing lines to fuse and split, was presented to us by D. Gaiotto, in the context of generalizing [25] to theories with gauge group factors $\mathrm{SU}(N)$ with $N>2$.
} 


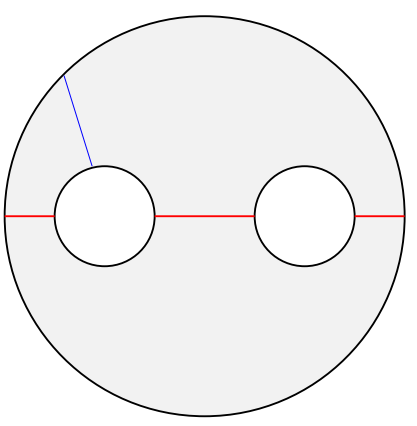

$\ell_{12}$

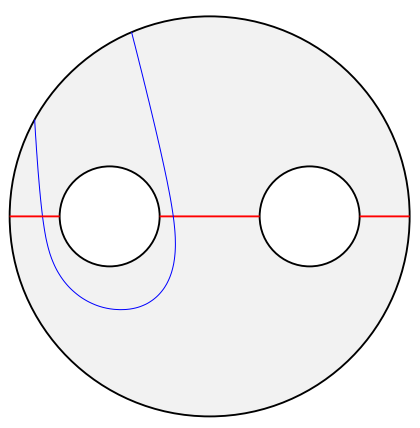

$\ell_{11}$

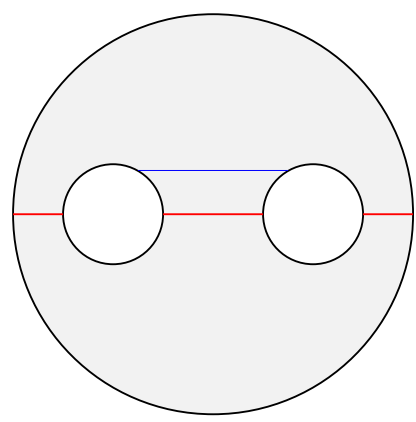

$\ell_{23}$

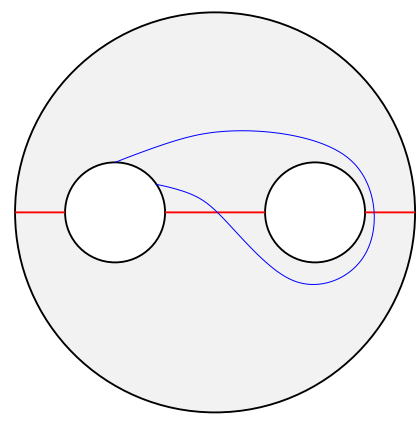

$\ell_{22}$

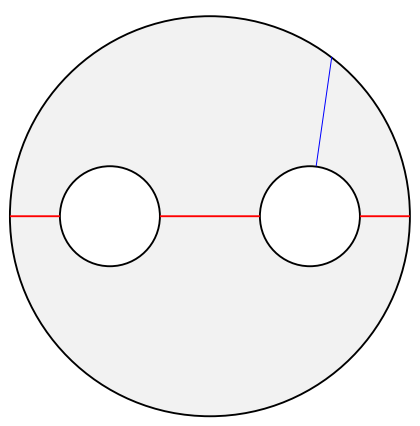

$\ell_{13}$

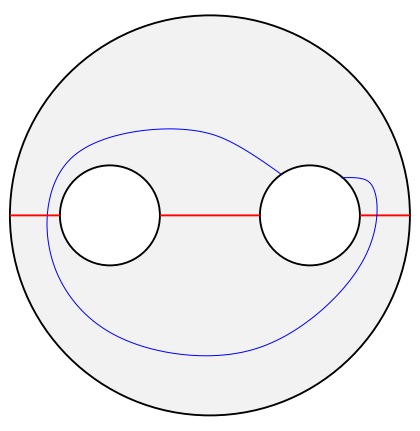

$\ell_{33}$

Figure 5. Basic arcs on a pair-of-pants

the pairs of pants will start and end on the upper half of the boundary circles as shown in figure 5 , and follow one of these six basic paths.

Given the number of lines crossing the three boundaries of the pair of pants, $p_{1}, p_{2}$ and $p_{3}$, there is a unique collection of non-intersection arcs as in figure 5 with these number of endpoints.

If $p_{i}>p_{j}+p_{k}$, then we can use

$$
\frac{1}{2}\left(p_{i}-p_{j}-p_{k}\right) \ell_{i i}+p_{j} \ell_{i j}+p_{k} \ell_{i k}
$$

On the other hand, if $p_{j}+p_{k} \geq p_{i}$ for all permutations of $\{1,2,3\}$, then we can use

$$
\frac{1}{2}\left(p_{1}+p_{2}-p_{3}\right) \ell_{12}+\frac{1}{2}\left(p_{1}+p_{3}-p_{2}\right) \ell_{13}+\frac{1}{2}\left(p_{2}+p_{3}-p_{1}\right) \ell_{23} .
$$

Instead of carrying the $V_{1,2}$ field along an arc and then on to another pair of pants, we want to treat all the arcs on this pair of pants in unison. For that we replace the $p_{i}$ lines crossing the boundary $i$ by a single line for the degenerate field $V_{1, p+1}$. We then consider the splitting and joining of these operators from one boundary to the other according to the multiplicities of the $\ell_{i j}$ curves.

More concretely we start by introducing pairs of degenerate fields $V_{1, p_{i}+1}$ at the center of the $i^{\text {th }}$ edge which are connected to the original graph through a handle carrying the identity state. If $q_{i} \neq 0$, we split off a $V_{1, q_{i}+1}$ field, braid it around and refuse it back to 
a pair of $V_{1, p_{i}+1}$. This prescription is valid for $p_{i} \geq q_{i}$, otherwise we start with a pair of $V_{1, q_{i}+1}$, braid one around, split each to a pair $V_{1, p_{i}+1}$ and $V_{1, q_{i}-p_{i}+1}$ and combine the latter two and project the intermediate state to the identity.

We then have exactly the situation envisioned above, where at each boundary of a pair of pants there is an extra degenerate field with $V_{1, p_{i}+1}$. They are split and fused with each-other according to the degeneracies of the $\ell_{i j}$ lines and projected on to the identity.

This prescription can be used in the calculation of the loop operators discussed in the preceding subsections. In the case of the one-punctured torus, there is a single pair of pants on which $p_{1}=p_{2}=1$ and $p_{3}=0$. The relevant curve is $\ell_{12}$. We define first the operator $\hat{w}_{1,0}$ as a map from the space of conformal blocks $\mathcal{C}\left(C_{g, n}\right)$ to the space of conformal blocks with an extra pair of degenerate fields $\hat{\mathcal{C}}\left(C_{g, n+2}\right)$, which is the inverse of the canonical identification and then places them at the two sides of the glued pairs of pants. In our case the two degenerate fields end up on the edges labeled 1 and 2 of the three-leg graph representing the pair of pants.

We next consider the operator $\hat{\ell}_{12}$ acting on the enlarged space of conformal blocks on a single pair of pants, moves one degenerate field from boundary number 2 to boundary number 1, combines the two and projects down back to the original space of conformal blocks.

Explicitly we can illustrate the action of $\hat{\ell}_{12}$ by the fusion and projection steps (in the case of the torus we also identify $\alpha_{1}=\alpha_{2}$, but for now we keep it generic)

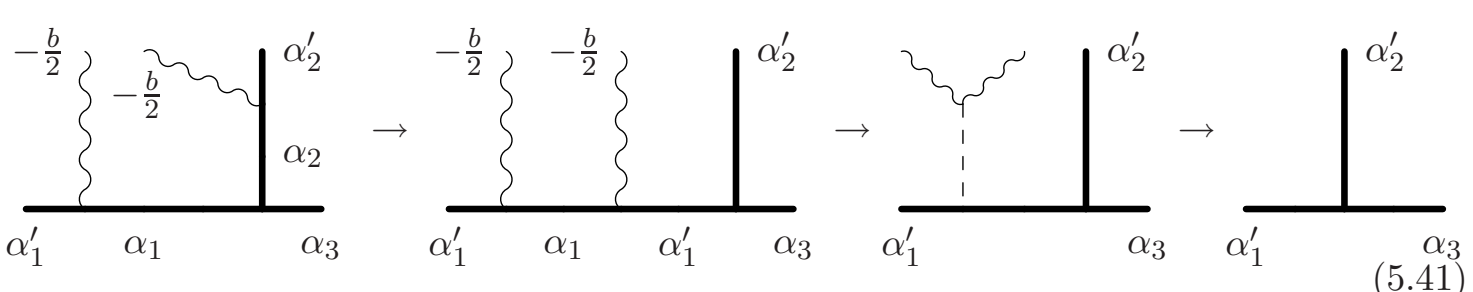

Taking $\alpha_{1}^{\prime}=\alpha_{1}-s_{1} b / 2$ and $\alpha_{2}^{\prime}=\alpha_{2}-s_{2} b / 2$ with $s_{i}= \pm 1$ we have for the renormalized conformal blocks $\mathcal{G}(5.7)$

$$
\left[\hat{\ell}_{12} \cdot \hat{\mathcal{G}}\right]_{\alpha_{1}, \alpha_{2}, \alpha_{3}}=G_{\alpha_{2}, \alpha_{1}^{\prime}}^{-1}\left[\begin{array}{cc}
-\frac{b}{2} & \alpha_{2}^{\prime} \\
\alpha_{1} & \alpha_{3}
\end{array}\right] G_{\alpha_{1}, 0}\left[\begin{array}{cc}
-\frac{b}{2} & -\frac{b}{2} \\
\alpha_{1}^{\prime} & \alpha_{1}^{\prime}
\end{array}\right] \mathcal{G}_{\alpha_{1}^{\prime}, \alpha_{2}^{\prime}, \alpha_{3}} .
$$

Using the expressions (B.5) and (B.7) in appendix B this is

$$
\left[\hat{\ell}_{12} \cdot \hat{\mathcal{G}}\right]_{\alpha_{1}, \alpha_{2}, \alpha_{3}}=s_{1} s_{2} \frac{\sin \left(\pi b\left(\alpha_{3}-\frac{Q}{2}+s_{1} s_{2} \frac{Q}{2}-s_{1}\left(\alpha_{2}-\frac{Q}{2}\right)-s_{2}\left(\alpha_{1}-\frac{Q}{2}\right)\right)\right.}{\sin \left(2 \pi b^{2}\right)} \mathcal{G}_{\alpha_{1}^{\prime}, \alpha_{2}^{\prime}, \alpha_{3}}
$$

Similarly one can define analogous operators $\hat{\ell}_{23}$ and $\hat{\ell}_{31}$ which are given by permuting the indices, and these operations for other degenerate fields.

Using this operator we can immediately reproduce the 't Hooft loop on the onepunctured torus calculated in section 5.3. We take $\alpha_{1}=\alpha_{2}=\alpha$ and $\alpha_{3}=m$. Then we have to act on the conformal block with $\hat{w}_{1,0}$, which will introduce $s=s_{1}=s_{2}$ and then act by $\hat{\ell}_{12}$

$$
\mathcal{G}_{\alpha, \alpha_{3}} \quad \rightarrow \quad\left[\hat{w}_{1,0} \cdot \mathcal{G}\right]_{\alpha, m}=\sum_{s= \pm} \hat{\mathcal{G}}_{\alpha^{\prime}, \alpha, m}^{s} \quad \rightarrow \quad\left[\hat{\ell}_{12} \cdot \hat{w}_{1,0} \cdot \mathcal{G}\right]_{\alpha, m}=\sum_{s= \pm} \mathcal{G}_{\alpha^{\prime}, m}
$$


Using (5.43) and including the action of $\hat{w}_{1,0}$ (B.6) we get

$$
\begin{aligned}
{\left[\hat{\ell}_{12} \cdot \hat{w}_{1,0} \cdot \mathcal{G}\right]_{\alpha, m} } & =\sum_{s= \pm} \frac{\sin (\pi b(m-s(2 \alpha-Q)))}{\sin \left(2 \pi b^{2}\right)} \times s \frac{\sin \left(\pi b^{2}\right)}{\sin (\pi b(2 \alpha-Q))} \mathcal{G}_{\alpha-s b / 2, m} \\
& =\sum_{s= \pm} \frac{\sin \left(\pi b^{2}\right)}{\sin \left(2 \pi b^{2}\right)} \frac{\sin (\pi b(2 \alpha-Q-s m)}{\sin (\pi b(2 \alpha-Q))} \mathcal{G}_{\alpha-s b / 2, m}^{s} .
\end{aligned}
$$

This indeed agrees precisely with (5.25).

In the case of the four-punctured sphere there are two pairs of pants. The loop considered in section 5.4 has $p=2$ on one boundary of each pair of pants. In our present approach of doing the calculation with the highest possible degenerate fields we need to create a pair of $V_{1,3}$ fields with $\hat{w}_{2,0}$.

Then on each pair of pants we need to act by the operator $\hat{\ell}_{11}$ which takes the $V_{1,3}$ degenerate field attached to the first edge, replace it by a pair of $V_{1,2}$ fields, transports one of them around the second edge, fusing it back to the other degenerate field and projecting on to the identity

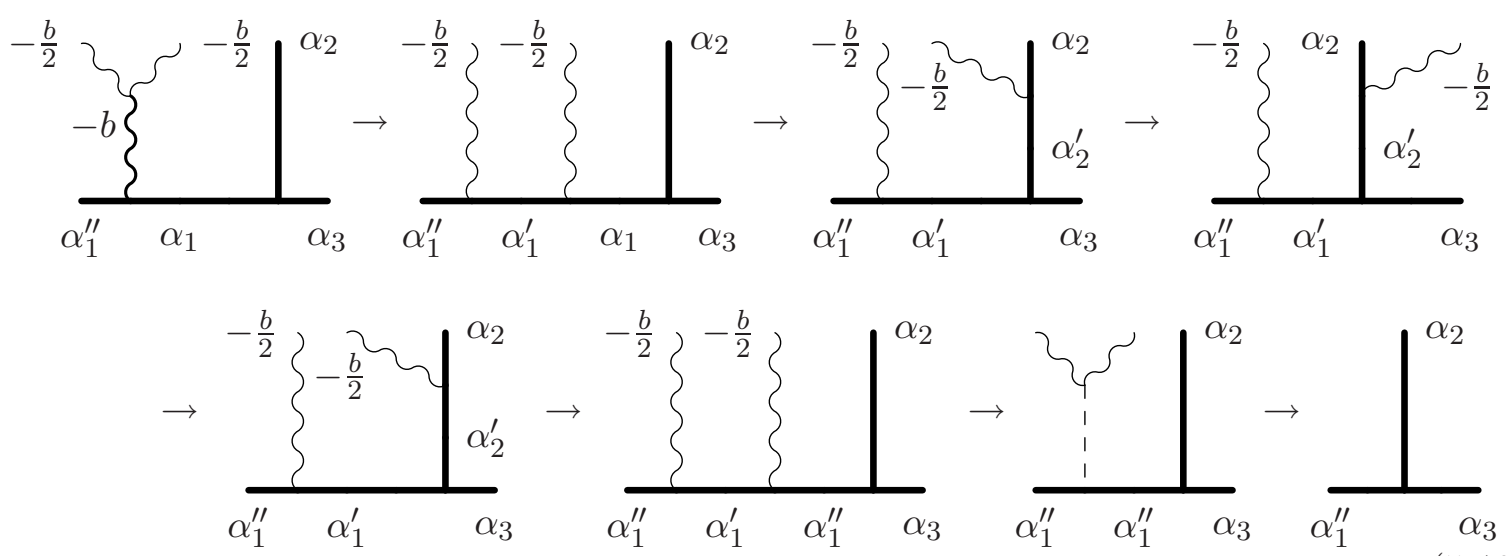

To write it down we take $\alpha_{1}^{\prime}=\alpha_{1}-s_{1} b / 2, \alpha_{1}^{\prime \prime}=\alpha_{1}^{\prime}-s_{1}^{\prime} b / 2$ and $\alpha_{2}^{\prime}=\alpha_{2}-s_{2} b / 2$. Then we have

$$
\begin{aligned}
{\left[\hat{\ell}_{11} \cdot \hat{\mathcal{G}}\right]_{\alpha_{1}, \alpha_{2}, \alpha_{3}}=} & \sum_{s_{1}, s_{2}= \pm} G_{-b, \alpha_{1}^{\prime}}^{-1}\left[\begin{array}{cc}
-\frac{b}{2} & -\frac{b}{2} \\
\alpha_{1}^{\prime \prime} & \alpha_{1}
\end{array}\right] G_{\alpha_{1} \alpha_{2}^{\prime}}\left[\begin{array}{cc}
-\frac{b}{2} & \alpha_{2} \\
\alpha_{1}^{\prime} & \alpha_{3}
\end{array}\right]\left(B_{\alpha_{2}^{\prime}}^{-\frac{b}{2}, \alpha_{2}}\right)^{2} \\
\times & G_{\alpha_{2}^{\prime} \alpha_{1}^{\prime \prime}}^{-1}\left[\begin{array}{cc}
-\frac{b}{2} & \alpha_{2} \\
\alpha_{1}^{\prime} & \alpha_{3}
\end{array}\right] G_{\alpha_{1}^{\prime} 0}\left[\begin{array}{cc}
-\frac{b}{2} & -\frac{b}{2} \\
\alpha_{1}^{\prime \prime} & \alpha_{1}^{\prime \prime}
\end{array}\right] \mathcal{G}_{\alpha_{1}^{\prime \prime}, \alpha_{2}, \alpha_{3}} \\
= & \sum_{s_{1}, s_{2}= \pm} G_{+, s_{1}}\left[\begin{array}{cc}
\alpha_{1} & -\frac{b}{2} \\
\alpha_{1}^{\prime \prime} & -\frac{b}{2}
\end{array}\right] G_{s_{2},-s_{1}}\left[\begin{array}{cc}
\alpha_{2} & -\frac{b}{2} \\
\alpha_{3} & \alpha_{1}^{\prime}
\end{array}\right] e^{\pi b i\left(Q+s_{2}\left(2 \alpha_{2}-Q\right)\right)} \\
\times & G_{s_{1}^{\prime} s_{2}}\left[\begin{array}{cc}
\alpha_{1}^{\prime} & -\frac{b}{2} \\
\alpha_{3} & \alpha_{2}
\end{array}\right] G_{-,-s_{1}^{\prime}}\left[\begin{array}{cc}
-\frac{b}{2} & -\frac{b}{2} \\
\alpha_{1}^{\prime \prime} & \alpha_{1}^{\prime \prime}
\end{array}\right] \mathcal{G}_{\alpha_{1}^{\prime \prime}, \alpha_{2}, \alpha_{3}}
\end{aligned}
$$

To find an explicit expression one uses (B.5) and (B.7) and performs the sum over $s_{1}$ and $s_{2}$. To simplify the final expression it is convenient to consider separately the cases with $\alpha_{1}^{\prime \prime}=\alpha_{1}-k b / 2$ with $k=-2,0,2$. For $k= \pm 2$ we need to take $s_{1}=s_{1}^{\prime}= \pm 1$ while for $k=0$ we need to sum over the two possibilities of $s_{1}=-s_{1}^{\prime}$. The result is

$$
\left[\hat{\ell}_{11} \cdot \hat{\mathcal{G}}\right]_{\alpha_{1}, \alpha_{2}, \alpha_{3}}=-2 i e^{i \pi b^{2}} \frac{\sin \left(\pi b\left(\alpha_{1}+\alpha_{2}-\alpha_{3}-Q\right)\right) \sin \left(\pi b\left(\alpha_{1}-\alpha_{2}+\alpha_{3}-Q\right)\right)}{\sin \left(2 \pi b^{2}\right)} \mathcal{G}_{\alpha_{1}-b, \alpha_{2}, \alpha_{3}}
$$




$$
\begin{aligned}
& +2 i e^{i \pi b^{2}} \frac{\cos \left(\pi b\left(2 \alpha_{1}-b\right)\right) \cos \left(\pi b\left(2 \alpha_{2}-b\right)\right)-\cos \left(\pi b^{2}\right) \cos \left(\pi b\left(2 \alpha_{3}-b\right)\right)}{\sin \left(2 \pi b^{2}\right)} \mathcal{G}_{\alpha_{1}, \alpha_{2}, \alpha_{3}} \\
& +2 i e^{i \pi b^{2}} \frac{\sin \left(\pi b\left(\alpha_{1}+\alpha_{2}+\alpha_{3}-Q\right)\right) \sin \left(\pi b\left(\alpha_{1}-\alpha_{2}-\alpha_{3}+Q\right)\right)}{\sin \left(2 \pi b^{2}\right)} \mathcal{G}_{\alpha_{1}+b, \alpha_{2}, \alpha_{3}}
\end{aligned}
$$

To complete the calculation of the loop operator on the four punctured sphere we have to include the action of $\hat{w}_{2,0}$ (B.12), then take a pair of these $\ell_{11}$ operators, one for each pair of pants, and sum over the three different values of $k$.

This gives the same as the loop calculated in section 5.4 without the term on the last line of (5.33). Indeed as stated before, this last term corresponds to the contribution from the identity state, or the trivial 't Hooft loop and the expression we derive here should correspond to the loop operator in an irreducible representation.

The operators $\hat{\ell}_{22}$ and $\hat{\ell}_{33}$ can be defined in a similar fashion, as can the operators for higher degenerate fields.

\section{The Liouville-Teichmüller theory}

Liouville theory has a dual representation in terms of a quantum mechanical problem, the quantum theory of the Teichmüller spaces. We first explain the basic quantization problem for the Teichmüller spaces based on their natural symplectic structures. We also explain how to describe the length of geodesics on a Riemann surface as functions on Teichmüller space. Then we recall the connection between Liouville theory and quantum Teichmüller theory established in [28, 29]. Quantum Teichmüller theory possesses canonical observables, namely geodesic length operators. We show that they are precisely the Liouville loop operators we introduced in section 4 , up to rescaling.

\subsection{Classical Liouville-Teichmüller theory on Riemann surfaces}

\subsubsection{Complex-analytic picture}

Teichmüller space is the space of deformations of the complex structure on a topological surface $\Sigma_{g, n}$. For each complex structure on a surface $\Sigma_{g, n}$ there exists a unique metric of the form

$$
d s^{2}=e^{\varphi} d z d \bar{z}
$$

which has constant negative curvature. A metric of the form (6.1) will have constant negative curvature $-8 \pi \mu b^{2}$ iff it satisfies the classical Liouville equation

$$
\partial \bar{\partial} \varphi=2 \pi \mu b^{2} e^{\varphi} .
$$

The classical Liouville field $\varphi$ is related to the quantum field $\phi$ used earlier by $\varphi=2 b \phi$. We may therefore identify the Teichmüller space $\mathrm{T}_{g, n}$ with the space of solutions of the Liouville equation on the surface $\Sigma_{g, n}$.

We will consider the space of all solutions to the classical Liouville equation on a surface $\Sigma_{g, n}$ as the phase space $\mathrm{T}_{g, n}$ we are aiming to quantize. The Liouville action functional $S^{\mathrm{cl}}[\varphi]$,

$$
S^{\mathrm{cl}}[\varphi]=\frac{1}{8 \pi} \int_{C_{g, n}} d^{2} z\left(\frac{1}{2}\left(\partial_{a} \varphi\right)^{2}+8 \pi \mu b^{2} e^{\varphi}\right)+[\text { boundary terms }],
$$




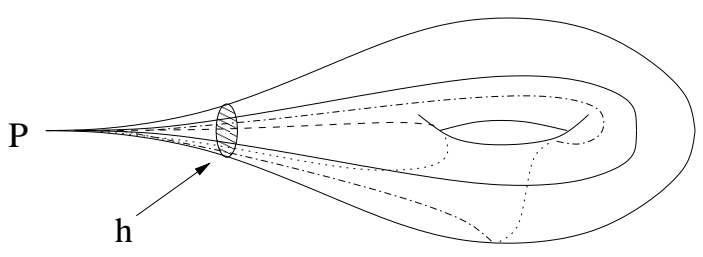

Figure 6. Triangulation of the once-punctured torus.

with choice of boundary terms as given in [44, 45], defines a natural symplectic form $\omega$ on $\mathrm{T}_{g, n}$,

$$
\omega=2 \pi i \partial \bar{\partial} S^{\mathrm{cl}}
$$

where $\partial, \bar{\partial}$ are the holomorphic and anti-holomorphic components of the de Rham differential on $\mathrm{T}_{g, n}$ respectively. The symplectic form $\omega$ coincides $[44,45]$ with the Weil-Petersson symplectic form, which is natural from the point of view of Teichmüller theory, $\omega=\omega_{\mathrm{WP}}$. The problem to quantize Liouville theory on a surface $\Sigma_{g, n}$ is therefore equivalent to the problem of quantizing Teichmüller space with Poisson-bracket given by the Weil-Petersson symplectic structure.

\subsubsection{Hyperbolic picture}

Uniformization offers a complementary picture on the Teichmüller spaces: Instead of the complex structure let us now focus on the associated hyperbolic metric as the relevant geometric structure on $C_{g, n}$. Natural coordinates for the Teichmüller spaces can then be defined in terms of the lengths of geodesics on the surface with hyperbolic metric. These coordinates offer two big advantages concerning the intended quantization of the Teichmüller spaces: They are real, so one may expect to find self-adjoint operators as their quantum representatives, and the Poisson bracket associated to the symplectic form (6.4) becomes very simple (linear) if the class of geodesics is suitably chosen.

A particularly useful set of coordinates was introduced by R. Penner in [46]. They can be defined for Riemann surfaces that have at least one puncture. One may assume having triangulated the surface by geodesics that start and end at the punctures. As an example we have drawn in figure 6 a triangulation $\tau$ of the once-punctured torus. The length of these geodesics will be infinite. In order to regularize this divergence one may introduce one horocycle around each puncture and measure only the length of the segment of a geodesic that lies between the horocycles. Assigning to an edge $e$ its regularized length $l_{e}$ gives coordinates for the so-called decorated Teichmüller spaces. These are fiber spaces over the Teichmüller spaces which have fibers that parameterize the choices of the "cut-offs" as introduced by the horocycles.

A closely related set of coordinates for the Teichmüller spaces themselves was introduced by Fock in [47]. The coordinate $z_{e}$ associated to an edge $e$ of a triangulation can be expressed in terms of the Penner-coordinates via $z_{e}=l_{a}+l_{c}-l_{b}-l_{d}$, where $a, b, c$ and $d$ label the other edges of the triangles that have $e$ in its boundary as indicated in figure 7 . 


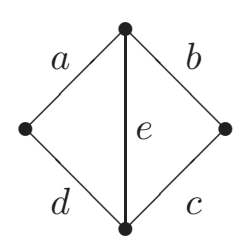

Figure 7. The labeling of the edges

Instead of triangulations of the Riemann surfaces it is often convenient to consider the corresponding fat graphs, which are defined by putting a trivalent vertex into each triangle and by connecting these vertices such that the edges of the triangulation are in one-to-one correspondence to the edges of the fat-graph.

\subsubsection{Symplectic structure in the hyperbolic picture}

As already mentioned, the Teichmüller spaces carry a natural symplectic form, called Weil-Petersson symplectic form. We are therefore dealing with a family of phase-spaces, one for each topological type of the Riemann surfaces. One of the crucial virtues of the Penner-coordinates is the fact that the Weil-Petersson symplectic form has a particularly simple expression in these coordinates [48]. The corresponding Poisson-brackets are in fact constant for Fock's variables $z_{e}[47]$,

$$
\left\{z_{e}, z_{e^{\prime}}\right\}=n_{e, e^{\prime}}, \quad \text { where } n_{e, e^{\prime}} \in\{-2,-1,0,1,2\} .
$$

The value of $n_{e, e^{\prime}}$ depends on how edges $e$ and $e^{\prime}$ are imbedded into a given fat graph. If $e$ and $e^{\prime}$ don't have a common vertex at their ends, or if one of $e, e^{\prime}$ starts and ends at the same vertex then $n_{e, e^{\prime}}=0$. In the case that $e$ and $e^{\prime}$ meet at two vertices one has $n_{e, e^{\prime}}=2$ (resp. $n_{e, e^{\prime}}=-2$ ) if $e^{\prime}$ is the first edge to the right ${ }^{30}$ (resp. left) of $e$ at both vertices, and $n_{e, e^{\prime}}=0$ otherwise. In all the remaining cases $n_{e, e^{\prime}}=1$ (resp. $n_{e, e^{\prime}}=-1$ ) if $e^{\prime}$ is the first edge to the right (resp. left) of $e$ at the common vertex.

If one considers a surface $\Sigma_{g, n}$ with genus $g$ and $n$ boundary components one will find $n$ central elements in the Poisson-algebra defined by (6.5). These central elements $c_{a}, a=1, \ldots, n$ are constructed as $c_{a}=\sum_{e \in E_{a}} z_{e}$, where $E_{a}$ is the set of edges in the triangulation that emanate from the $a^{\text {th }}$ boundary component. The value of $c_{a}$ gives the geodesic length of the $a^{\text {th }}$ boundary component [47].

\subsubsection{Length functions}

Having identified the phase space of Liouville theory on $C_{g, n}$ with the Teichmüller space $\mathrm{T}_{g, n}$ suggests to look for interesting observables. The lengths of closed geodesics, considered as functions on $\mathrm{T}_{g, n}$, are certainly interesting objects from the geometric point of view.

A nice feature of the Fock coordinates is that they lead to a particularly simple way to reconstruct the length functions $l_{\gamma}(P)$ corresponding to the point $P$ in Teichmüller space that is parametrized by the variables $z_{e}(P)$. Assume given a path $\varpi_{\gamma}$ on the fat graph homotopic to a simple closed curve $\gamma$ on $C_{g, n}$. Let the edges be labelled $e_{i}, i=1, \ldots, r$

\footnotetext{
${ }^{30}$ The orientation is induced by the imbedding of the fat-graph into the surface.
} 
according to the order in which they appear on $\varpi_{\gamma}$, and define $\sigma_{i}$ to be 1 if the path turns left at the vertex that connects edges $e_{i}$ and $e_{i+1}$, and to be equal to -1 otherwise. Consider the following matrix,

$$
\mathrm{X}_{\gamma}=\mathrm{V}^{\sigma_{r}} \mathrm{E}\left(z_{e_{r}}\right) \ldots \mathrm{V}^{\sigma_{1}} \mathrm{E}\left(z_{e_{1}}\right)
$$

where the matrices $\mathrm{E}(z)$ and $\mathrm{V}$ are defined respectively by

$$
\mathrm{E}(z)=\left(\begin{array}{cc}
0 & +e^{+\frac{z}{2}} \\
-e^{-\frac{z}{2}} & 0
\end{array}\right), \quad V=\left(\begin{array}{cc}
1 & 1 \\
-1 & 0
\end{array}\right) .
$$

One may then calculate the hyperbolic length of the closed geodesic isotopic to $c$ via [47]

$$
L_{\gamma} \equiv 2 \cosh \left(\frac{1}{2} l_{\gamma}\right)=\left|\operatorname{tr}\left(\mathrm{X}_{\gamma}\right)\right| .
$$

\subsection{Kähler quantization}

A quick way to anticipate that the solution of the problem to quantize the Teichmüller spaces is related to conformal field theory goes as follows, see [28] for a more detailed discussion. Let us restrict to the case $g=0$ with $n$ conical singularities for notational simplicity. Complex analytic coordinates for the moduli space $\mathcal{M}_{0, n}$ of Riemann surfaces with genus 0 and $n$ punctures are given by the positions of the conical singularities $z_{1}, \ldots, z_{n-3}$ if the remaining three are assumed to be located at 0,1 and $\infty$, respectively

$$
\mathcal{M}_{0, n}=\left\{\left(z_{1}, \ldots, z_{n-3}\right) ; z_{i} \neq 0,1 \text { and } z_{i} \neq z_{j} \text { for } i \neq j\right\}
$$

The corresponding canonically conjugate momenta w.r.t. $\omega$ can be defined as

$$
C_{i}=-\partial_{z_{i}} S^{\mathrm{cl}} .
$$

Indeed, since $S^{\mathrm{cl}}$ is the Kähler potential for $\omega$ we have the following Poisson-brackets

$$
\left\{z_{i}, z_{j}\right\}=0=\left\{C_{i}, C_{j}\right\}, \quad\left\{z_{i}, C_{j}\right\}=\frac{1}{2 \pi i} \delta_{i j} .
$$

An observation from $[44,49]$ which is important for us is the fact that the momenta $C_{i}$ parameterize the classical energy-momentum tensor $T_{\varphi}(z) \equiv-\frac{1}{4}\left(\varphi_{z}\right)^{2}+\frac{1}{2} \varphi_{z z}$ associated to a solution $\varphi$ of (6.2). Indeed, it can be shown (see e.g. [50]) that $T_{\varphi}(z)$ can be expanded as

$$
T_{\varphi}(z)=\sum_{i=1}^{n-1}\left(\frac{\delta_{i}}{\left(z-z_{i}\right)^{2}}+\frac{C_{i}}{z-z_{i}}\right) .
$$

The asymptotic behavior of $T_{\varphi}(z)$ near $z=\infty$ may be represented as

$$
T_{\varphi}(z)=\frac{\delta_{n}}{z^{2}}+\frac{C_{n}}{z^{3}}+O\left(z^{-4}\right) .
$$

The so-called accessory parameters $C_{i}$ are highly nontrivial functions on the moduli space $\mathcal{M}_{0, n}$ which are restricted by the relations

$$
\sum_{i=1}^{n-1} C_{i}=0, \quad \sum_{i=1}^{n-1}\left(z_{i} C_{i}+\delta_{i}\right)=\delta_{n}, \quad \sum_{i=1}^{n-1}\left(z_{i}^{2} C_{i}+2 \delta_{i} z_{i}\right)=C_{n} .
$$


In analogy to the coherent state representation of quantum mechanics it is then natural to consider a quantization scheme in which states are represented by holomorphic multivalued wave-functions

$$
\Psi(z)=\langle z \mid \Psi\rangle, \quad z=\left(z_{1}, \ldots, z_{n-3}\right),
$$

such that the operators $z_{i}$ corresponding to the classical observables $z_{i}$ are represented as multiplication operators, $\mathrm{z}_{i} \Psi(z)=z_{i} \Psi(z)$. The state $|z\rangle$ introduced in (6.15) is thereby identified as an analog of a coherent state (eigenstate of the "creation operators" $z_{i}$ ) in quantum mechanics.

The operators $C_{i}$ associated to the momenta $C_{i}$ conjugate to $z_{i}$ should be represented by the differential operators $b^{2} \partial_{z_{i}}$ in such a representation. The energy-momentum tensor $T_{\varphi}$ would then be represented by the operator $b^{2} \mathrm{~T}(z)$, where

$$
\mathbf{T}(z)=\sum_{i=1}^{n-1}\left(\frac{\Delta_{\alpha_{i}}}{\left(z-z_{i}\right)^{2}}+\frac{1}{z-z_{i}} \frac{\partial}{\partial z_{i}}\right) .
$$

We have introduced the quantum conformal dimensions $\Delta_{\alpha_{i}}$ which are related to the $\delta_{i}$ by $\delta_{i}=b^{2} \Delta_{i}+\mathcal{O}\left(b^{2}\right)$. This makes the space of holomorphic wave-functions obtained in the Kähler quantization of the Teichmüller spaces into a module over the ring of holomorphic differential operators on $\mathrm{T}_{0, n}$.

This should be compared with the well-known statement that the Virasoro conformal blocks can be represented by means of holomorphic functions on $\mathrm{T}_{0, n}$ denoted by $\left\langle T(x) \Psi_{\alpha_{n}}\left(z_{n}\right) \ldots \Psi_{\alpha_{1}}\left(z_{1}\right)\right\rangle$ which form a module over the ring of holomorphic differential operators on $\mathrm{T}_{0, n}$ as is expressed by the conformal Ward indentities

$$
\left\langle T(x) \Psi_{\alpha_{n}}\left(z_{n}\right) \ldots \Psi_{\alpha_{1}}\left(z_{1}\right)\right\rangle=\sum_{i=1}^{n-1}\left(\frac{\Delta_{\alpha_{i}}}{\left(x-z_{i}\right)^{2}}+\frac{1}{x-z_{i}} \frac{\partial}{\partial z_{i}}\right)\left\langle\Psi_{\alpha_{n}}\left(z_{n}\right) \ldots \Psi_{\alpha_{1}}\left(z_{1}\right)\right\rangle .
$$

Comparison of (6.16) and (6.17) suggests that wave-functions in the Kähler quantization of $\mathrm{T}_{0, n}$ can be identified with Virasoro conformal blocks. The precise correspondence will be reviewed in subsection 6.5 below.

\subsection{Quantization in a real polarization}

It is very difficult to describe the quantization of the length operators directly in the Kähler quantization above. The basic reason is that the relation between complex analytic coordinates for $\mathrm{T}_{g, n}$ and the geodesic length functions involves uniformizing the surface $C_{g, n}$, which generically is highly transcendental. It turns out to be possible, however, to bypass this difficulty by first studying the quantum theory obtained by directly quantizing the Teichmüller spaces using real coordinates, and then establishing the link of this quantization scheme with the Kähler quantization.

\subsubsection{Algebra of observables and Hilbert space}

The simplicity of the Poisson brackets (6.5) makes part of the quantization quite simple. To each edge $e$ of a triangulation of a Riemann surface $C_{g, n}$ associate a quantum operator 
$\mathrm{z}_{e}$. The algebra of observables $\mathcal{A}\left(C_{g, n}\right)$ will be the algebra with generators $\mathrm{z}_{e}$, relations

$$
\left[\mathbf{z}_{e}, \mathbf{z}_{e^{\prime}}\right]=2 \pi i b^{2}\left\{z_{e}, z_{e^{\prime}}\right\}
$$

and hermiticity assignment $\mathbf{z}_{e}^{\dagger}=\mathrm{z}_{e}$. The algebra $\mathcal{A}\left(C_{g, n}\right)$ has a center with generators $\mathrm{c}_{a}$, $a=1, \ldots, n$ defined by $\mathrm{c}_{a}=\sum_{e \in E_{a}} \mathrm{z}_{e}$, where $E_{a}$ is the set of edges in the triangulation that emanates from the $a^{\text {th }}$ boundary component. The representations of $\mathcal{A}\left(C_{g, n}\right)$ that we are going to consider will therefore be such that the generators $c_{a}$ are represented as the operators of multiplication by real positive numbers $l_{a}$. Geometrically one may interpret $l_{a}$ as the geodesic length of the $a^{\text {th }}$ boundary component [47]. The vector $l=\left(l_{1}, \ldots, l_{n}\right)$ of lengths of the boundary components will figure as a label of the representation $\pi\left(C_{g, n}, \Lambda\right)$ of the algebra $\mathcal{A}\left(C_{g, n}\right)$.

To complete the definition of the representation $\pi\left(C_{g, n}, \Lambda\right)$ by operators on a Hilbert space $\mathcal{H}\left(C_{g, n}\right)$ one just needs to find linear combinations $\mathrm{x}_{1}, \ldots, \mathrm{x}_{3 g-3+n}$ and $\mathrm{p}_{1}, \ldots, \mathrm{p}_{3 g-3+n}$ of the $\mathrm{z}_{e}$ that satisfy $\left[\mathrm{p}_{i}, \mathrm{x}_{j}\right]=(2 \pi i)^{-1} \delta_{i j}$. The representation of $\mathcal{A}\left(C_{g, n}, \Lambda\right)$ on $\mathcal{H}\left(C_{g, n}\right):=$ $L^{2}\left(R^{3 g-3+n}\right)$ is defined by choosing the usual Schrödinger representation for the $\mathrm{x}_{i}, \mathrm{p}_{i}$.

It is very important to make sure that the resulting quantum theory does not depend on the underlying triangulation in an essential way. This can be done by constructing a family of unitary operators $\mathrm{U}_{\tau_{2}, \tau_{1}}$ that describe the change of representation when passing from the quantum theory associated to triangulation $\tau_{1}$ to the one associated to $\tau_{2}[29,51-53]$.

\subsection{Geodesic length operators}

Length operators were first studied in the pioneering works [51, 54]. On first sight the problem looks rather simple. We may observe that the classical expression for $L_{\gamma} \equiv$ $2 \cosh \frac{1}{2} l_{\gamma}$ as given by formula 6.8 is a linear combination of monomials in the variables $e^{ \pm \frac{z_{e}}{2}}$ of a very particular form,

$$
L_{\gamma}=\sum_{\nu \in \mathcal{F}} C_{\tau, \gamma}(\nu) e^{(\nu, z)}, \quad(\nu, z)=\sum_{e} \nu_{e} z_{e}
$$

where the summation is taken over a finite set $\mathcal{F}$ of vectors $\nu \in\left(\frac{1}{2} \mathbb{Z}\right)^{3 g-3+2 n}$ with halfinteger components $\nu_{e}$. The coefficients $C_{\tau, \gamma}(\nu)$ are positive integers. In the quantum case one is interested in the definition of length operators $L_{\tau, \gamma}$ which should be representable by expressions of the form,

$$
\mathrm{L}_{\tau, \gamma}=\sum_{\nu \in \mathcal{F}} C_{\tau, \gamma}(\nu): e^{(\nu, \mathrm{z})}:_{b}=\sum_{\nu \in \mathcal{F}} C_{\tau, \gamma}^{b}(\nu) e^{(\nu, \mathrm{z})}, \quad(\nu, \mathrm{z})=\sum_{e} \nu_{e} \mathbf{z}_{e}
$$

where the notation $: \mathrm{O}:{ }_{b}$ indicates use of a quantum ordering prescription. In the second expression we have moved the effect of the ordering into a quantum deformation $C_{\tau, \gamma}^{b}(\nu)$ of the coefficients $C_{\tau, \gamma}(\nu)$.

Note, on the other hand, that the following additional properties seem to be indispensable if one wants to interpret an operator of the general form (6.20) as the quantum counterpart of the functions $L_{\tau, \gamma}=2 \cosh \frac{1}{2} l_{\gamma}$ : 
(a) Spectrum: $\mathrm{L}_{\tau, \gamma}$ is simple and takes values in $[2, \infty)$. This is necessary and sufficient for the existence of an operator $\mathrm{I}_{\tau, \gamma}$ - the geodesic length operator - such that $\mathrm{L}_{\tau, \gamma}=$ $2 \cosh \frac{1}{2} \mathrm{I}_{\gamma}$.

\section{(b) Independence of triangulation:}

$$
\mathrm{U}_{\tau_{2} \tau_{1}}^{-1} \cdot \mathrm{L}_{\tau_{1}, \gamma} \cdot \mathrm{U}_{\tau_{2} \tau_{1}}^{-1}=\mathrm{L}_{\tau_{2}, \gamma},
$$

where $\mathrm{U}_{\tau_{2} \tau_{1}}$ is the unitary operator relating the representation associated to triangulation $\tau_{1}$ to the one associated to $\tau_{2}$.

Property (b) ensures that the collection of length operators $\mathrm{L}_{\tau, \gamma}$ associated to the different triangulations $\tau$ ultimately defines an operator $\mathrm{L}_{\gamma}$ that is independent of the triangulation. It was observed in $[51,54]$ that the deformation of the coefficients $C_{\tau, \gamma}^{b}(\nu)$ which is necessary for having the properties (a) and (b) can be quite nontrivial in general, in the sense that it can not be obtained from a simple ordering prescription.

A general construction of length operators which fulfils the requirements above was given in [29]. This construction coincides with the one in $[51,54]$ whenever both can be applied.

\subsubsection{The length representation}

It can be shown that the length operators associated to non-intersecting simple closed curves commute with each other. This together with the self-adjointness of the length operators allows one to introduce bases of eigenfunctions for the length operators.

One gets one such basis for each pants decomposition of $C_{g, n}$. A key result for the connection between quantum Liouville and quantum Teichmüller theory is that for each marking $\sigma$ there exists a basis for $\mathcal{H}_{g, n}$ spanned by $|l\rangle_{\sigma}, l=\left(l_{1}, \ldots, l_{3 g-3+n}\right)$ which obeys the factorization rules of conformal field theory [29]. This means in particular that for any pair $\sigma_{2}, \sigma_{1}$ of markings one can always decompose the unitary transformation $\mathrm{V}_{\sigma_{2} \sigma_{1}}$ which relates the representation corresponding to marking $\sigma_{1}$ to the one corresponding to $\sigma_{2}$ as a product of operators which represent the elementary fusion, braiding and S-moves introduced in section 5.1. The unitary transformation $\mathrm{V}_{\sigma_{2} \sigma_{1}}$ can be represented as an integral operator of the form

$$
\left|l_{2}\right\rangle_{\sigma_{2}}=\int d \mu\left(l_{1}\right) V_{\sigma_{2} \sigma_{1}}\left(l_{2}, l_{1}\right)\left|l_{1}\right\rangle_{\sigma_{1}} .
$$

The explicit expressions for the kernel $V_{\sigma_{2} \sigma_{1}}\left(l_{2}, l_{1}\right)$ are known for the cases where $\sigma_{2}$ and $\sigma_{1}$ differ by one of the elementary moves.

\subsection{Relation between length representation and Kähler quantization}

When discussing the more general case of $g \geq 0$ in the following we will use the gluing parameters $q_{i}$ as generalizations of the holomorphic coordinates $z_{i}$ used in 6.2. The coherent states $|q\rangle$ are the eigenstates of the operators $\mathrm{q}_{i}$ corresponding to the holomorphic coordinates $q_{i}$, and the wave-functions are holomorphic functions ${ }^{31} \Psi(q)=\langle\Psi \mid q\rangle$ on $\mathrm{T}_{g, n}$.

\footnotetext{
${ }^{31}$ More precisely sections of a projective line bundle on $\mathrm{T}_{g, n}$.
} 
The change of representation from length representation to the holomorphic representation is described by means of the matrix elements

$$
\Psi_{l}^{\sigma}(q)=\langle l \mid q\rangle_{\sigma}
$$

The following characterization of these matrix elements was obtained in [28]:

$$
\Psi_{l}^{\sigma}(q)=\mathcal{G}_{\alpha, E}^{(\sigma)}(q)
$$

where $\mathcal{G}_{\alpha, E}^{(\sigma)}(q)$ is the Liouville conformal block ${ }^{32}(2.5)$ associated to a marking $\sigma$ with external representations labeled by $E=\left(m_{1}, \ldots, m_{n}\right)$ and fixed intermediate dimensions given by the parameters $\alpha=\left(\alpha_{1}, \ldots, \alpha_{3 g-3+n}\right)$. These parameters are related to the lengths $c_{a}$ of the boundary components and to the lengths $l_{i}$ around the curves defining the pants decomposition respectively as

$$
m_{a}=\frac{Q}{2}+i \frac{c_{a}}{4 \pi b}, \quad \alpha_{i}=\frac{Q}{2}+i \frac{l_{i}}{4 \pi b}=\frac{Q}{2}+a_{i}
$$

where $a=1, \ldots, n$ and $i=1, \ldots, 3 g-3+n$. The main nontrivial result underlying (6.23) is the fact that both sides transform in the same way under a change of marking $\sigma$. Comparing also the asymptotic behavior at the boundaries of $\mathrm{T}_{g, n}$ then allows one to conclude that the holomorphic functions $\Psi_{l}^{\sigma}(q)$ and $\mathcal{G}_{\alpha, E}^{(\sigma)}(q)$ must coincide [28].

Let us then consider the resolution of the identity, the so-called Bergman kernel, defined as

$$
B\left(\bar{q}, q^{\prime}\right)=\int d \mu(l)\langle q \mid l\rangle_{\sigma \sigma}\left\langle l \mid q^{\prime}\right\rangle=\int d \mu(l)\left(\Psi_{l}^{\sigma}(q)\right)^{*} \Psi_{l}^{\sigma}\left(q^{\prime}\right) .
$$

Comparing with (2.6) we see that the correlation functions of Liouville theory on a Riemann surface $C_{g, n}$ can be represented as

$$
\left\langle\prod_{a=1}^{n} V_{m_{a}}\right\rangle_{C_{g, n}}=B(\bar{q}, q) .
$$

The relation (6.23) can be generalized to a direct relation between the geodesic length operators $\mathrm{L}_{\gamma}$ from quantum Teichmüller theory and the Liouville loop operators $\mathcal{L}(\gamma)$,

$$
\left(\mathrm{L}_{\gamma} \Psi_{l}^{\sigma}\right)(q)=\kappa\left(\mathcal{L}(\gamma) \mathcal{G}_{\alpha, E}^{(\sigma)}\right)(q), \quad \kappa=2 \cos \pi b Q .
$$

This relation is easily verified by considering the case where $\gamma$ is one of the curves defining the pants decomposition associated to $\sigma$, say $\gamma_{i}$. Both the operators $\mathrm{L}_{\gamma_{i}}$ and $\kappa \mathcal{L}\left(\gamma_{i}\right)$ are then diagonal in this basis and are represented by multiplication with $2 \cosh \left(l_{i} / 2\right)$ and $2 \cosh \pi b\left(2 \alpha_{i}-Q\right)$ respectively (5.15), which are indeed equal (6.24). The validity of (6.27) in general then follows from the fact that both sides transform the same way under changes of the marking.

\footnotetext{
${ }^{32}$ We indicate here that the dependence on the complex structure $q$ which is suppressed in the rest of the text.
} 
We finally arrive at the following interpretation of the loop operator expectation values within quantum Teichmüller theory.

$$
\kappa\langle\mathcal{L}(\gamma)\rangle_{C_{g, n}}=\left\langle q\left|\mathrm{~L}_{\gamma}\right| q\right\rangle
$$

This means that the expectation value of the Liouville loop operator has the geometric interpretation as the expectation value of the geodesic length operator $L_{\gamma}$ in the coherent state $|q\rangle$ which corresponds to a fixed complex structure parameterized by $q$. It therefore solves the natural quantum counterpart of the classical problem to calculate the geodesic length of $\gamma$ in the metric associated by uniformization to the complex structure parameterized by $q$.

\section{Algebra of loop operators in Liouville-Teichmüller theory}

Given a set of operators, it is very natural to study the algebra formed by them. For loop operators in gauge theory, this problem goes back to 't Hooft's original paper [55] where magnetic loop operators were first introduced. There it was found that Wilson and 't Hooft loops in a spatial slice at constant time, acting on the Hilbert space of gauge theory, give rise to interesting commutation relations. The relations, which will be referred as the 't Hooft commutation relations, encode information about the electric and magnetic charges of the loop operators as well as how the loops are linked in the spatial slice. Another interesting algebraic property to consider is the operator product expansion (OPE) of loop operators. The OPE captures the decomposition of the product of two loop operators defined on two curves in spacetime into a basis of loop operators in gauge theory in the limit where two operators are brought together.

In previous sections we have explicitly computed the action of Liouville loop operators supported on curves on the Riemann surface on the conformal blocks. The action involves multiplication by functions of $\alpha_{i}, m_{a}$ as well as shifts of $\alpha_{i}$, where $\alpha_{i}$ and $m_{a}$ label the internal and external edges of the trivalent graph characterizing the conformal block. Such multiplication and shifts can be thought of as operators acting on the space of conformal blocks, and form an algebra that can be computed explicitly in examples. The description of this algebra in terms of quantum Teichmüller theory is equivalent to that in Liouville theory, as discussed in section 6 .

In this section we study the algebra of loop operators in Liouville theory and provide a physical interpretation of the algebra in gauge theory. First we explain the fundamental relation satisfied by the Liouville loop operators. The relation, known as the quantum skein relation, completely determines the algebra of loop operators.

We then show that the commutation relations that follow from the quantum skein relation at $b=1$ are precisely the ones found by 't Hooft for the gauge theory loop operators that are Hopf-linked in a three-dimensional constant time slice.

Furthermore, the quantum skein relations can also be viewed as a prediction for the OPE of loop operators in gauge theory. We compare this to the OPE of loop operators in the gauge theory deduced from S-duality, by generalizing an argument given for a special case in [56]. We point out that the Liouville loop operators that appear in the product of 

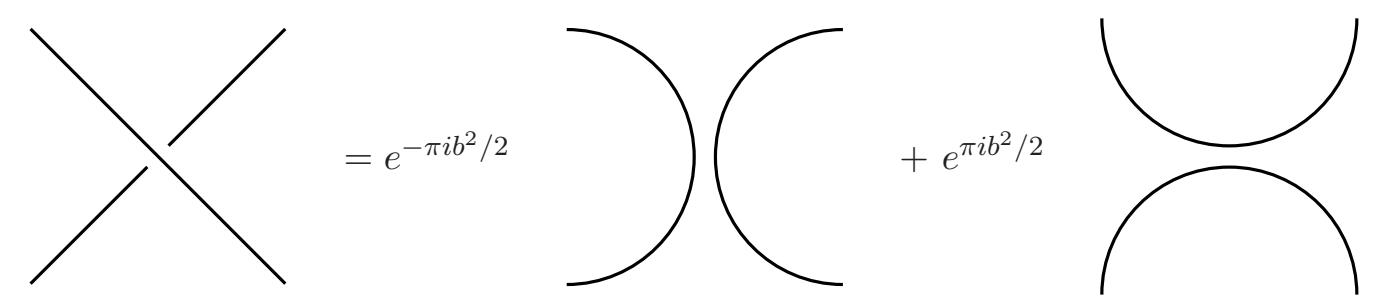

Figure 8. A graphical expression of the quantum skein relation among the length operators.

two loop operators agree, but that the numerical prefactors do not, and propose that the difference has to do with whether the loops are Hopf-linked or not. We illustrate these general results, by considering the concrete expressions derived earlier for the Liouville loop operators in $\mathcal{N}=2^{*}$ super Yang-Mills theory and the $\mathcal{N}=2 \mathrm{SU}(2)$ gauge theory with $N_{F}=4$.

\subsection{Quantum skein relation}

It is known from quantum Teichmüller theory $[51,54]$ that the geodesic length operators form a closed algebra with basic relation being the so-called quantum skein relation, see [57] for a nice review of the necessary results and references. As explained in section 6.5, the Liouville loop operator $\mathcal{L}(\gamma)$ corresponding to a geodesic $\gamma$ is exactly the geodesic length operator $\mathrm{L}_{\gamma}$, up to rescaling by the constant $\kappa=2 \cos (\pi b Q)$, when we identify the Hilbert space of quantum Teichmüller theory with the space of Liouville conformal blocks. It follows that the Liouville loop operators satisfy the quantum skein relation.

To explain the relation, let us first consider a pair of connected closed non-selfintersecting curves $\gamma$ and $\gamma^{\prime}$ on the Riemann surface $C_{g, n}$ that intersect at one point. There are two distinct ways to remove the intersection and reconnect the two curves as shown in figure 8 , where the line on top in the left hand side is identified with $\gamma$. Let us denote by $\gamma \star \gamma^{\prime}$ and $\gamma \circ \gamma^{\prime}$ the two resulting curves corresponding to the first and second terms in the right hand side of figure 8, respectively. Then the four length operators $L_{\gamma}, L_{\gamma^{\prime}}, L_{\gamma \star \gamma^{\prime}}$ and $\mathrm{L}_{\gamma \circ \gamma^{\prime}}$ satisfy the quantum skein relation

$$
\mathrm{L}_{\gamma} \cdot \mathrm{L}_{\gamma^{\prime}}=e^{-\pi i b^{2} / 2} \mathrm{~L}_{\gamma \star \gamma^{\prime}}+e^{\pi i b^{2} / 2} \mathrm{~L}_{\gamma \circ \gamma^{\prime}} .
$$

It is also possible to derive the relation for the Liouville loop operators directly using the moves described in previous sections. ${ }^{33}$ When $\gamma$ and $\gamma^{\prime}$ have more than one intersection,

\footnotetext{
${ }^{33}$ The composition of operators $\mathcal{L}(\gamma) \cdot \mathcal{L}\left(\gamma^{\prime}\right)$ can be realized as an operation that splits the identity into two degenerate fields $V_{1,2}\left(z_{1}\right)$ and $V_{1,2}\left(z_{2}\right)$ at the intersection, moves $V_{1,2}\left(z_{1}\right)$ around along $\gamma$, fuses them back to the identity, splits it again, moves $V_{1,2}\left(z_{1}\right)$ around along $\gamma^{\prime}$, and finally fuses them back to the identity.

The operator $\mathcal{L}\left(\gamma \star \gamma^{\prime}\right)$ on the other hand is an operation that splits the identity into $V_{1,2}\left(z_{1}\right)$ and $V_{1,2}\left(z_{2}\right)$ at the intersection, moves $V_{1,2}\left(z_{1}\right)$ around along $\gamma$ back to the intersection point, moves $V_{1,2}\left(z_{1}\right)$ around along $\gamma^{\prime}$, and finally fuses them back to the identity. Here we have assigned orientations to $\gamma$ and $\gamma^{\prime}$ such that they consistently define an orientation of $\gamma \star \gamma^{\prime}$.

Similarly the operator $\mathcal{L}_{\gamma \circ \gamma^{\prime}}$ can be represented as an operation that splits the identity into $V_{1,2}\left(z_{1}\right)$ and $V_{1,2}\left(z_{2}\right)$ at the intersection, moves $V_{1,2}\left(z_{1}\right)$ around along $\gamma$ back to the intersection point, moves $V_{1,2}\left(z_{2}\right)$ around along $\gamma^{\prime}$, and finally fuses them back to the identity. Note that it is $z_{2}$, not $z_{1}$, that is moved around along $\gamma^{\prime}$.

Writing out the fusion matrices corresponding to these moves, one can derive the quantum skein relation.
} 


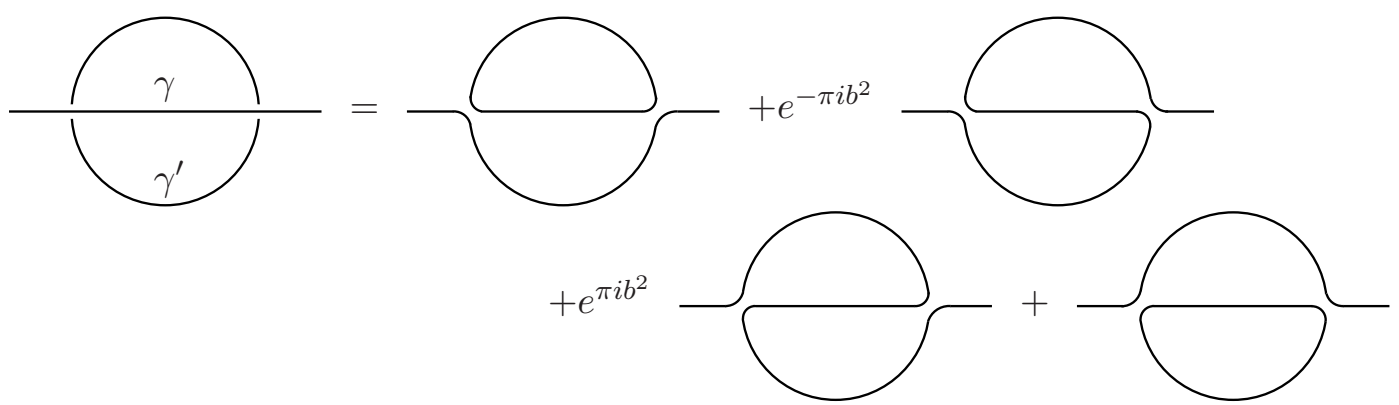

Figure 9. The quantum skein relation for two curves $\gamma$ and $\gamma^{\prime}$ that intersect at two points. $L_{\gamma} L_{\gamma^{\prime}}$ on the left hand side is expanded in length operators for non-intersecting curves. Note that the ordering of $\gamma$ and $\gamma^{\prime}$ matters in this relation.

one should apply the skein relation locally for all the intersections at the same time as in figure 9. A contractable curve is assigned the value $-e^{-\pi i b^{2}}-e^{\pi i b^{2}}=2 \cos (\pi b Q)$. See [58] for more examples.

Let us note that there is a natural basis of length/loop operators in which we can apply the quantum skein relation. The operators in this basis are in one-to-one correspondence with the homotopy classes of non-self-intersecting closed curves on the Riemann surface $C_{g, n}$ labeled by Dehn-Thurston parameters. Let $\gamma$ be such a curve. Then $\gamma$ in general has more than one connected components. We denote by $K_{I}$ the number of components whose homotopy class is that of a curve $\gamma^{(I)}$. Then the operator that corresponds to $\gamma$ is given by

$$
\mathrm{L}_{\gamma} \equiv \prod_{I} \mathrm{~L}_{\gamma^{(I)}}^{K_{I}}
$$

Now let $\gamma$ and $\gamma^{\prime}$ be not-necessarily-connected simple closed curves. The components of $\gamma$ generically intersect those of $\gamma^{\prime}$ at several points. By repeatedly using the quantum skein relation above, one can express the product of any operators of the form (7.2) as a sum of operators of the same form.

\subsection{Comparison with gauge theory}

\subsubsection{The commutation relations}

It follows that when $b=1$, we have

$$
\mathrm{L}_{\gamma} \cdot \mathrm{L}_{\gamma^{\prime}}=(-1)^{\#\left(\gamma \cap \gamma^{\prime}\right)} \mathrm{L}_{\gamma^{\prime}} \cdot \mathrm{L}_{\gamma}
$$

where \# $\left(\gamma \cap \gamma^{\prime}\right)$ is the number of intersections between $\gamma$ and $\gamma^{\prime}$.

We wish to provide a gauge theory interpretation for the commutation relations (7.3) satisfied by the length operators. As we now explain, this is precisely the commutation relation satisfied by loop operators that are linked in a constant time slice, as originally studied by 't Hooft [55]. 
Let us briefly review the commutation relations found by 't Hooft. It suffices for us to consider a single gauge group $\mathrm{SU}(2)$. Let $L_{0,1}$ be the pure Wilson loop along curve 1 and $L_{1,0}$ the pure 't Hooft loops along curve 2. The two curves 1 and 2 are taken to be lie in the three-dimensional space and we assume that they are Hopf-linked. ${ }^{34}$ We are interested in the canonical quantization of $\mathrm{SU}(2)$ gauge theory with matter that is neutral under the center $\mathbb{Z}_{2}$ of the gauge group. The product

$$
L_{0,1} L_{1,0}
$$

corresponds to inserting the Wilson loop $L_{0,1}$ at time $\epsilon>0$ and the 't Hooft loop $L_{1,0}$ at time zero. Likewise,

$$
L_{1,0} L_{0,1}
$$

corresponds to inserting the Wilson loop $L_{0,1}$ at time $-\epsilon<0$ and the 't Hooft loop $L_{1,0}$ at time zero. The 't Hooft loop $L_{1,0}$ is defined by a background field configuration that is singular along the curve 2 at time zero. The singular configuration is such that at any time $t>0$, there is a non-trivial background holonomy along a spatial curve that links the curve 2. ${ }^{35}$ Thus the Wilson loop $L_{0,1}$ inserted at time $\epsilon>0$ picks up the holonomy -1 relative to the Wilson loop inserted at time $-\epsilon<0$, implying the commutation relation

$$
L_{0,1} L_{1,0}=-L_{1,0} L_{0,1}
$$

for the loop operators acting on the Hilbert space of gauge theory.

The argument generalizes to more than one $\mathrm{SU}(2)$ factor in the gauge group and higher charges. Let $\gamma$ and $\gamma^{\prime}$ be connected simple closed geodesics with Dehn-Thurston parameters $d$ and $d^{\prime}$, respectively. We can choose a pants decomposition where $\gamma$ is a boundary of a pair of pants. In other words, the gauge theory loop operator $L_{d}$ is a pure Wilson loop in, say the first $\mathrm{SU}(2)$ gauge group. In this case we have the commutation relation

$$
L_{d} L_{d^{\prime}}=(-1)^{p_{1}^{\prime}} L_{d^{\prime}} L_{d},
$$

where $p_{1}^{\prime}$ is the magnetic charge of $L_{d^{\prime}}$ in the first gauge group. Clearly $p_{1}^{\prime}$ is the intersection number of $\gamma$ and $\gamma^{\prime}$.

Thus we can write the commutation relation in a frame-independent form as

$$
L_{d} L_{d^{\prime}}=(-1)^{\#\left(\gamma \cap \gamma^{\prime}\right)} L_{d^{\prime}} L_{d}
$$

We see that the loop operators in gauge theory, supported on spacetime loops that are Hopf-linked obey precisely the same commutation relations - the 't Hooft commutation relations - as those for the corresponding geodesic length/Liouville loop operators at $b=1$. This observation will lead to a conjecture and a definite prediction, which we will formulate in section 8 .

\footnotetext{
${ }^{34}$ 't Hooft's analysis is applicable in any gauge theory, also without supersymmetry. To relate it to Liouville theory we need Hopf-linked loops in $\mathcal{N}=2$ gauge theory which share some supersymmetry. Luckily this is the case for a pair of non-intersecting loops on $S^{3}$, as was shown for $\mathcal{N}=4 \mathrm{SYM}$ in $[59,60]$. The same argument carries over to our case.

${ }^{35}$ In Euclidean spacetime, the spatial 't Hooft loop is obtained by inserting a magnetic monopole along the loop. We can let the family of Dirac strings extend in the positive time direction. Thus at any positive time, the Dirac string singularity is along the spatial loop, and going around it produces a phase (sign).
} 


\subsubsection{The operator product expansion}

As remarked at the end of section 7.1, there is a natural basis of geodesic length/Liouville loop operators labeled by not-necessarily-connected closed geodesics. In this basis, the quantum skein relation allows one to decompose the product of operators into the sum of operators. The correspondence of gauge theory and Liouville loop operators proposed in section 4 then leads us to view the quantum skein relation as providing a rule for how to perform the corresponding decomposition, namely the operator product expansion (OPE) of loop operators in gauge theory.

We observed above that the commutation relations at $b=1$ that follow from the quantum skein relation are precisely those for gauge theory loop operators on Hopf-linked spacetime loops. At this value of $b$, the quantum skein relation further requires that the gauge theory loop operators satisfy the OPE

$$
2 L_{d} L_{d^{\prime}}=-i L_{d \star d^{\prime}}+i L_{d \circ d^{\prime}}
$$

where we have denoted by $d, d^{\prime}, d \star d^{\prime}$ and $d \circ d^{\prime}$ the sets of Dehn-Thurston parameters of the curves $\gamma, \gamma^{\prime}, \gamma \star \gamma^{\prime}$ and $\gamma \circ \gamma^{\prime}$, respectively. The OPE (7.9) then is a prediction for the gauge theory loop operators, to be discussed fully in section 8 .

In fact we can compute the OPE of loop operators in the setup where the loops are not linked, by generalizing an argument used in [56] for $\mathcal{N}=4$ super Yang-Mills. Since the loops are not linked, the results do not necessarily have to agree with the Liouville loop operators. Interestingly, as we show below, the OPE of the non-linked loop operators exactly matches the classical skein relations, namely the relation (7.1) at $b=0$.

In our $\mathcal{N}=2$ gauge theories, S-duality provides a specific identification of the algebra of loop operators with the group algebra of

$$
\left(\Lambda_{m} \times \Lambda_{w}\right) / \mathcal{W}
$$

where $\Lambda_{w} \simeq \mathbb{Z}^{3 g-3+n}$ is the weight lattice and $\mathcal{W} \simeq\left(\mathbb{Z}_{2}\right)^{3 g-3+n}$ is the Weyl group, both associated with the gauge group $G=\mathrm{SU}(2)^{3 g-3+n}$. We have also defined the lattice $\Lambda_{m}$ of magnetic charges $\left(p_{1}, \ldots, p_{3 g-3+n}\right) \in \mathbb{Z}^{3 g-3+n}$ subject to the Dirac quantization condition (2.12), so that (7.10) is the space of allowed Dehn-Thurston parameters $d=$ $\left(p_{i}, q_{i}\right){ }^{36}$ Let us represent the group algebra of $\Lambda_{m}$ by polynomials of $X_{i}, X_{i}^{-1}$, and the group algebra of $\Lambda_{w}$ by polynomials of $Y_{i}, Y_{i}^{-1}$ where $i=1, \ldots, 3 g-3+n$. There is an obvious action of the Weyl group that in particular exchanges $X_{i}$ with $X_{i}^{-1}$ and $Y_{i}$ with $Y_{i}^{-1}$. The variable $Y_{i}$ carrying the electric charge is interpreted as the Abelian Wilson loop with unit charge for the $i^{\text {th }} \mathrm{SU}(2)$ group, in the Coulomb branch of the gauge theory where the $\mathrm{SU}(2)$ is broken to a U(1) subgroup. Since the Wilson loop $L_{d_{i}}$ with the DehnThurston parameters all vanishing except for $q_{i}=1$ is in the fundamental representation of the $i^{\text {th }} \mathrm{SU}(2)$ gauge group, which has two weights of opposite signs, we associate to $L_{d_{i}}$ the polynomial $\left(Y_{i}+Y_{i}^{-1}\right) / 2$ :

$$
L_{d_{i}} \mapsto\left(Y_{i}+Y_{i}^{-1}\right) / 2 .
$$

\footnotetext{
${ }^{36}$ The Weyl group action replaces the conditions that $p_{j} \geq 0$, and that if $p_{j}=0$ then $q_{j} \geq 0$.
} 
The factor of $1 / 2$ reflects the normalization of $L_{d_{i}}$ in (5.17). For 't Hooft loops, a similar interpretation is more subtle and involves "monopole bubbling" [22]. As explained in [56], however, S-duality allows us to determine the polynomials corresponding to an arbitrary non-linked loop operator $L_{d}$ associated to a connected simple closed geodesic, and hence in fact a general closed non-self-intersecting geodesic which always consists of connected components. This is because as in the case of $\mathcal{N}=4$ super Yang-Mills with $\mathrm{SU}(2)$ gauge group considered in [22], in the theories $\mathcal{T}_{g, n}$ any loop operator can be transformed to a pure Wilson loop by a duality transformation, which acts on the Dehn-Thurston parameters [25]. Thus to an arbitrary loop operator corresponding to a connected simple closed geodesic $\gamma_{d}$ with $d=\left(p_{i}, q_{i}\right)$, we associate a polynomial as

$$
L_{d} \mapsto \frac{1}{2}\left(\prod_{i} Y_{i}^{p_{i}} X_{i}^{q_{i}}+\prod_{i} Y_{i}^{-p_{i}} X_{i}^{-q_{i}}\right)
$$

Given this identification of loop operators and the group algebra, the OPE of non-linked loop operators is simply given by the multiplication rules of the group algebra.

Let us now demonstrate that the algebra of non-linked gauge theory loop operators is precisely that of the geodesic length/Liouville loop operators. This is done by considering two connected simple closed geodesics $\gamma$ and $\gamma^{\prime}$ that share a single intersection point. As before we choose a pants decomposition such that $\gamma$ is the closed geodesic corresponding to the first gauge $\mathrm{SU}(2)$ group. Let $d=\left(0, \delta_{i 1}\right)$ and $d^{\prime}=\left(p_{i}^{\prime}, q_{i}^{\prime}\right)$ be the vectors of DehnThurston parameters for $\gamma$ and $\gamma^{\prime}$, respectively. Then the OPE of the gauge theory loop operators $L_{d}$ and $L_{d^{\prime}}$ is given by decomposing the product of the associated polynomials:

$$
\begin{aligned}
& \left(Y_{1}+Y_{1}^{-1}\right)\left(\prod_{i} X_{i}^{p_{i}} Y_{i}^{q_{i}}+\prod_{i} X_{i}^{-p_{i}} Y_{i}^{-q_{i}}\right) \\
& \quad=\left(\prod_{i} X_{i}^{p_{i}} Y_{i}^{q_{i}+\delta_{i 1}}+\prod_{i} X_{i}^{-p_{i}} Y_{i}^{-q_{i}-\delta_{i 1}}\right)+\left(\prod_{i} X_{i}^{p_{i}} Y_{i}^{q_{i}-\delta_{i 1}}+\prod_{i} X_{i}^{-p_{i}} Y_{i}^{-q_{i}+\delta_{i 1}}\right)
\end{aligned}
$$

Thus we get loop operators associated with new Dehn-Thurston parameters $\left(p_{i}, q_{i}+\delta_{i 1}\right)$ and $\left(p_{i}, q_{i}-\delta_{i 1}\right)$. These are precisely the Dehn-Thurston parameters for $\gamma \star \gamma^{\prime}$ and $\gamma \circ \gamma^{\prime}$. Thus the OPE of the loop operators in this case is given by

$$
2 L_{d} L_{d^{\prime}}=L_{d \star d^{\prime}}+L_{d \circ d}
$$

This is exactly what follows from the classical skein relation, the skein relation for $b=0$.

We note that the operators which appear on the right hand side of (7.9), which is valid at $b=1$, are exactly the same as those appearing in the above equation at $b=0$, with modified coefficients. The fact that the same operators appear in the gauge theory calculation above supports our identification of loop operators in Liouville and the gauge theory and the conjectured form of the quantum OPE relations (7.9). It also lends some hope that the relation (7.9) at $b=1$ could be shown to hold for linked loop operators in the gauge theory. 


\subsection{Examples}

\subsection{1 $\mathcal{N}=2^{*}$ theory}

Let us study the algebra of loop operators in the $\mathcal{N}=2^{*} \mathrm{SU}(2)$ gauge theory. The Liouville theory realization was computed for Wilson loops in section 5.2, and for the minimal 't Hooft loop as well as some dyonic loops in section 5.3. We have the following Liouville loop operators expressed as multiplication and difference operators:

$$
\begin{aligned}
& \mathcal{L}\left(\gamma_{0,1}\right)=\frac{\cos (2 \pi b a)}{\cos (\pi b Q)}, \\
& \mathcal{L}\left(\gamma_{1, q}\right)=e^{-\frac{q}{2} \pi i b^{2}} \frac{\sin \left(\pi b^{2}\right)}{\sin \left(2 \pi b^{2}\right)}\left(e^{2 \pi i q b a} \frac{\sin (2 \pi b(a-m / 2))}{\sin (2 \pi b a)} e^{-\frac{b}{2} \partial_{a}}\right. \\
&\left.\quad+e^{-2 \pi i q b a} \frac{\sin (2 \pi b(a+m / 2))}{\sin (2 \pi b a)} e^{\frac{b}{2} \partial_{a}}\right) .
\end{aligned}
$$

One can check that the quantum skein relation (7.1) is satisfied. ${ }^{37}$ At $b=1$, the Liouville loop operators in (7.15) and (7.16) precisely satisfy the 't Hooft commutation relation

$$
\mathcal{L}\left(\gamma_{1, q}\right) \mathcal{L}\left(\gamma_{0,1}\right)=-\mathcal{L}\left(\gamma_{0,1}\right) \mathcal{L}\left(\gamma_{1, q}\right) .
$$

7.3.2 $\mathcal{N}=2 \mathrm{SU}(2)_{C}$ with $N_{F}=4$

Next, we consider $\mathcal{N}=2 \mathrm{SU}(2)$ super Yang-Mills with four hypermultiplets in the fundamental representation. The Wilson loops $\mathcal{L}\left(\gamma_{0,1}\right)$ have the same expressions as for $\mathcal{N}=2^{*}$ (7.15). The minimal 't Hooft loop $\mathcal{L}\left(\gamma_{2,0}\right)$ (recall that the magnetic charge $p$ has to be even by Dirac quantization) computed in (5.31) is given by

$$
\mathcal{L}\left(\gamma_{2,0}\right)=T_{2} e^{-b \partial_{\alpha}}+T_{0}+T_{-2} e^{b \partial_{\alpha}}
$$

with $T_{2}, T_{0}, T_{-2}$ given below (5.31). It is easy to check that the expected 't Hooft commutation is satisfied when $b=1$.

\section{Conclusions and discussion}

In this paper we have extended the correspondence [13] between the $\mathcal{N}=2$ theories $\mathcal{T}_{g, n}$ constructed by Gaiotto and Liouville theory on $C_{g, n}$ to include physical observables in the two theories. We have advanced a precise correspondence between gauge theory loop operators - for Wilson, 't Hooft and dyonic operators - and operators in the Liouville conformal field theory. ${ }^{38}$ We have found a one-to-one correspondence between loop operators in Liouville theory, which we have explicitly constructed, and gauge theory loop operators

$$
\text { loop operator in } \mathcal{T}_{g, n} \Longleftrightarrow \quad \text { Liouville loop operator in } C_{g, n} \text {. }
$$

\footnotetext{
${ }^{37}$ As discussed in section 3, we have chosen the precise phases of the Liouville loop operators so that they match those of the length operators.

${ }^{38}$ This is based on the one-to-one map found in [25] between the electric and magnetic charges of gauge theory loop operators and non-self-intersecting geodesics on the Riemann surface.
} 
Our construction of Liouville loop operators, which are supported on geodesics in $C_{g, n}$, is modular invariant, and provides a natural basis for non-local observables in the Liouville conformal field theory. Using the relation between Liouville conformal field theory and quantum Teichmüller theory, we have also found a mapping between loop operators in gauge theory and geodesic length operators in quantum Teichmüller theory.

We have shown that our computation of Liouville loop operators exactly reproduces the expectation value of Wilson loop operators in the $\mathcal{T}_{g, n}$ theories in [10]. The modular invariant construction of the Liouville loop operators provides us with a framework in which to calculate the expectation value of any dyonic operators by choosing a different pants decomposition of $C_{g, n}$, which maps the Wilson operator to a dyonic operator in the corresponding duality frame of $\mathcal{T}_{g, n}$. Our proposal, together with modular duality in Liouville theory [14-16] yields the expected action of the S-duality group $\Gamma\left(\mathcal{T}_{g, n}\right)$ on the loop operators in the $\mathcal{T}_{g, n}$ theory.

For specific examples of $\mathcal{T}_{g, n}$, we have explicitly computed the expectation value of 't Hooft and dyonic operators. We have considered $\mathcal{N}=2^{*}$ and $\mathcal{N}=2 \mathrm{SU}(2)$ gauge theory with $N_{F}=4$. Since no calculations of 't Hooft operators have been performed beyond the ones for $\mathcal{N}=4$ super Yang-Mills in [23, 24], our computations constitute predictions that would be interesting to confirm by a direct gauge theory analysis. A general algorithm for calculating 't Hooft and dyonic operators in an arbitrary $\mathcal{T}_{g, n}$ theory has also been proposed, whereby the computation can be broken into a sequence of operations that depends on the details of the theory and on the choice of loop operator.

We have also advanced a four dimensional field theory interpretation of the algebra of Liouville loop operators and geodesic length operators in quantum Teichmüller theory, which satisfy the quantum skein relation. The 't Hooft commutation relations of gauge theory loop operators [55], which describe the relations satisfied by loop operators that are Hopf-linked at a constant time slice in the four dimensional theory, have been identified with those that follow from the quantum skein relation of geodesic length operators at $b=1$. 't Hooft commutation relations of $\Longleftrightarrow \begin{aligned} & \text { commutation relations from the } \\ & \text { linked loop operators in } \mathcal{T}_{g, n}\end{aligned}$ quantum skein relation at $b=1$.

Furthermore, we have shown that the OPE of non-linked gauge theory loop operators, predicted by the proposed action of S-duality [25], precisely matches the classical skein relation of geodesic length operators (at $b=0)$.

OPE of commuting loop operators in $\mathcal{T}_{g, n} \Longleftrightarrow \quad$ classical skein relation.

We conjecture that gauge theory operators corresponding to the Liouville loop operators at $b=1$ are Hopf-linked with each other on the equatorial $S^{3}$ in $S^{4}$. Furthermore, we conjecture that the OPE coefficients of loop operators that correspond to Liouville loop operators at $b=1$ are given by the quantum skein relations at $b=1$.

An important open problem for the future is to extend Pestun's localization calculation [10] for the Wilson loops of $\mathcal{N}=2$ gauge theories to general dyonic loop operators. Our Liouville calculation of the expectation value of loop operators provides some hints. As we have shown, the expression for the 't Hooft operators is quite similar to that for 
Wilson loops. For 't Hooft loops, there are extra terms in the matrix integral with shifted arguments which one may speculate correspond to the contribution of the various weights characterizing the magnetic charges of the loop operator. This interpretation is consistent with the calculation of 't Hooft operators in $\mathcal{N}=4$ super Yang-Mills [23, 24], where it was found that there are multiple saddle point in the path integral. The physical interpretation of the extra saddle points has to do with monopole screening, whereby the singularity produced by the insertion of an 't Hooft operator is screened by regular monopoles, resulting in saddle points with weaker singularities and which is in one-to-one correspondence with the weights labeling the representation of the 't Hooft loop. Another important open problem is to provide a gauge theory interpretation of the prefactors multiplying the conformal blocks, which should correspond to the one loop determinant of fluctuations around the localization calculation for the 't Hooft loop.

It is of importance to attain a deeper understanding of the physical and mathematical foundations that underlie the gauge/Liouville correspondence. A possible point of contact is the parallel that exists between their Hilbert spaces and their inner products. The expression (1.3) for the Wilson loop expectation value that follows from localization in gauge theory on $S^{4}$ has a natural interpretation as the expectation value of the loop operator evaluated for the wave function defined by the Nekrasov instanton partition function. Let $\theta$ be the latitude on $S^{4}$. The instantons that contribute to the holomorphic part are localized at the North Pole $\theta=0$, and the anti-instantons at the South Pole $\theta=\pi$. The Wilson loop is located at an arbitrary latitude on the $S^{4}$, which without loss of generality can be taken to be in the "equator" $S^{3}$ at $\theta=\pi / 2$. Thus it is natural to interpret the holomorphic (anti-holomorphic) Nekrasov partition function as the wave function of a state, obtained by performing the gauge path-integral on the Northern (Southern) Hemisphere, in the BPS Hilbert space of the gauge theory canonically quantized on $S^{3}$.

It is then very suggestive to compare the resulting picture for the gauge theory loop expecation value with the form the expectation value takes in quantum Teichmüller theory, equation (6.28). It seems natural to propose identifying the Hilbert space of quantum Teichmüller theory with the BPS Hilbert space $\mathcal{H}_{\text {BPS }}$ and the coherent state $|q\rangle$ with the state in $\mathcal{H}_{\mathrm{BPS}}$ created by performing the gauge theory path-integral over the lower hemisphere. The geodesic length operators would thereby directly be related to the projection of the gauge theory loop operators onto the BPS Hilbert space.

Extensions of our results to higher rank gauge groups would also be worth pursuing. On the Riemann surface they involve conformal field theories with $\mathcal{W}$-symmetry [13, 61]. Increasing the rank of the gauge groups also admits an interesting generalization of quantum Teichmüller theory [62], which quantizes the moduli space of $\operatorname{SL}(N)$ flat connections for $N>2$ [63]. The corresponding gauge theories constructed out of the Riemann surface contain building blocks with no weakly coupled Lagrangian description [8]. Understanding the loop operators in the two dimensional conformal field theory as well as the generalization of the length operators in the quantization of the moduli space of $\mathrm{SL}(N)$ flat connections is bound to shed light in this interesting class of gauge theories.

Clearly it is of great interest to obtain a more direct interpretation of the correspondence between gauge theory and Liouville theory. Finding a more first principles interpre- 
tation may also provide hints of how to extend the two dimensional interpretation of four dimensional $\mathcal{N}=2$ gauge theories away from the choice of localization parameters satisfying $\epsilon_{1} / \epsilon_{2}=1$ relevant for the correspondence in Liouville. Progress in realizing the Nekrasov instanton partition function [11, 12] for arbitrary $\epsilon_{1}$ and $\epsilon_{2}$ have been made in topological string theory, where amplitudes sewn from a "refined" topological vertex [64, 65] were shown to reproduce Nekrasov's partition function. It would be interesting to incorporate the insights developed in that context to extend these exciting correspondences.

\section{Acknowledgments}

We would like to thank Harald Dorn, Davide Gaiotto, George Jorjadze, Natalia Saulina and Herman Verlinde for helpful discussions. N.D., J.G. and T.O. would like to thank the Benasque Center for Science for its hospitality. N.D is grateful to DESY Hamburg for its hospitality during the course of this work. Research at the Perimeter Institute is supported in part by the Government of Canada through NSERC and by the Province of Ontario through MRI. J.G. also acknowledges further support from an NSERC Discovery Grant and from and an ERA grant by the Province of Ontario. J.T. acknowledges support from the EC by the Marie Curie Excellence Grant MEXT-CT-2006-042695

\section{A Special functions}

\section{A.1 The function $\Gamma_{b}(x)$}

The function $\Gamma_{b}(x)$ is a close relative of the double Gamma function studied in [66, 67]. It can be defined by means of the integral representation

$$
\log \Gamma_{b}(x)=\int_{0}^{\infty} \frac{d t}{t}\left(\frac{e^{-x t}-e^{-Q t / 2}}{\left(1-e^{-b t}\right)\left(1-e^{-t / b}\right)}-\frac{(Q-2 x)^{2}}{8 e^{t}}-\frac{Q-2 x}{t}\right) .
$$

Important properties of $\Gamma_{b}(x)$ are

(i) Functional equation: $\Gamma_{b}(x+b)=\sqrt{2 \pi} b^{b x-\frac{1}{2}} \Gamma^{-1}(b x) \Gamma_{b}(x)$.

(ii) Analyticity: $\Gamma_{b}(x)$ is meromorphic,

$$
\text { poles: } x=-n b-m b^{-1}, n, m \in \mathbb{Z}^{\geq 0} .
$$

(iii) Self-duality: $\Gamma_{b}(x)=\Gamma_{1 / b}(x)$.

\section{A.2 The function $S_{b}(x)$}

The function $S_{b}(x)$ may be defined in terms of $\Gamma_{b}(x)$ as follows

$$
S_{b}(x)=\Gamma_{b}(x) / \Gamma_{b}(Q-x) .
$$

An integral that represents $\log S_{b}(x)$ is

$$
\log S_{b}(x)=\int_{0}^{\infty} \frac{d t}{t}\left(\frac{\sinh t(Q-2 x)}{2 \sinh b t \sinh b^{-1} t}-\frac{Q-2 x}{2 t}\right) .
$$


The most important properties for our purposes are

(i) Functional equation: $\quad S_{b}(x+b)=2 \sin \pi b x S_{b}(x)$.

(ii) Analyticity: $S_{b}(x)$ is meromorphic,

$$
\begin{aligned}
& \text { poles: } x=-\left(n b+m b^{-1}\right), n, m \in \mathbb{Z}^{\geq 0} . \\
& \text { zeros: } x=Q+\left(n b+m b^{-1}\right), n, m \in \mathbb{Z}^{\geq 0} .
\end{aligned}
$$

(iii) Self-duality: $S_{b}(x)=S_{1 / b}(x)$.

(iv) Inversion relation: $S_{b}(x) S_{b}(Q-x)=1$.

(v) Asymptotics: $\quad S_{b}(x) \sim e^{\mp \frac{\pi i}{2} x(x-Q)}$ for $\operatorname{Im}(x) \rightarrow \pm \infty$

(vi) Residue: $\operatorname{res}_{x=c_{b}} S_{b}(x)=(2 \pi)^{-1}$.

\section{A.3 $\Upsilon$ function}

The $\Upsilon$ may be defined in terms of $\Gamma_{b}$ as follows

$$
\Upsilon(x)^{-1} \equiv \Gamma_{b}(x) \Gamma_{b}(Q-x) .
$$

An integral representation convergent in the strip $0<\operatorname{Re}(x)<Q$ is

$$
\log \Upsilon(x)=\int_{0}^{\infty} \frac{d t}{t}\left[\left(\frac{Q}{2}-x\right)^{2} e^{-t}-\frac{\sinh ^{2}\left(\frac{Q}{2}-x\right) \frac{t}{2}}{\sinh \frac{b t}{2} \sinh \frac{t}{2 b}}\right] .
$$

Properties:

(i) Functional equation: $\Upsilon(x+b)=\frac{\Gamma_{b}(b x)}{\Gamma_{b}(1-b x)} b^{1-2 b x} \Upsilon(x)$.

(ii) Analyticity: $\Upsilon(x)$ is entire analytic,

$$
\text { zeros: } \begin{aligned}
x & =-\left(n b+m b^{-1}\right), n, m \in \mathbb{Z}^{\geq 0} . \\
x & =Q+\left(n b+m b^{-1}\right), n, m \in \mathbb{Z}^{\geq 0} .
\end{aligned}
$$

(iii) Self-duality: $\Upsilon(x)=\Upsilon_{1 / b}(x)$.

\section{B Fusion matrices}

In the following we will use the fusion coefficients involving some degenerate fields with $\alpha=-b / 2$. We define

$$
F_{s, s^{\prime}}\left[\begin{array}{cc}
\alpha_{3} & -\frac{b}{2} \\
\alpha_{4} & \alpha_{1}
\end{array}\right] \equiv F_{\alpha_{1}-\frac{s b}{2}, \alpha_{3}-\frac{s^{\prime} b}{2}}\left[\begin{array}{cc}
\alpha_{3} & -\frac{b}{2} \\
\alpha_{4} & \alpha_{1}
\end{array}\right]
$$

where $s, s^{\prime}= \pm$.

$$
\begin{aligned}
& F_{++}=\frac{\Gamma\left(b\left(2 \alpha_{1}-b\right)\right) \Gamma\left(b\left(b-2 \alpha_{3}\right)+1\right)}{\Gamma\left(b\left(\alpha_{1}-\alpha_{3}-\alpha_{4}+b / 2\right)+1\right) \Gamma\left(b\left(\alpha_{1}-\alpha_{3}+\alpha_{4}-b / 2\right)\right)} \\
& F_{+-}=\frac{\Gamma\left(b\left(2 \alpha_{1}-b\right)\right) \Gamma\left(b\left(2 \alpha_{3}-b\right)-1\right)}{\Gamma\left(b\left(\alpha_{1}+\alpha_{3}+\alpha_{4}-3 b / 2\right)-1\right) \Gamma\left(b\left(\alpha_{1}+\alpha_{3}-\alpha_{4}-b / 2\right)\right)} \\
& F_{-+}=\frac{\Gamma\left(2-b\left(2 \alpha_{1}-b\right)\right) \Gamma\left(b\left(b-2 \alpha_{3}\right)+1\right)}{\Gamma\left(2-b\left(\alpha_{1}+\alpha_{3}+\alpha_{4}-3 b / 2\right)\right) \Gamma\left(1-b\left(\alpha_{1}+\alpha_{3}-\alpha_{4}-b / 2\right)\right)} \\
& F_{--}=\frac{\Gamma\left(2-b\left(2 \alpha_{1}-b\right)\right) \Gamma\left(b\left(2 \alpha_{3}-b\right)-1\right)}{\Gamma\left(b\left(-\alpha_{1}+\alpha_{3}+\alpha_{4}-b / 2\right)\right) \Gamma\left(b\left(-\alpha_{1}+\alpha_{3}-\alpha_{4}+b / 2\right)+1\right)}
\end{aligned}
$$


Equivalently for the renormalized fusion coefficients we define

$$
G_{s, s^{\prime}}\left[\begin{array}{cc}
\alpha_{3} & -\frac{b}{2} \\
\alpha_{4} & \alpha_{1}
\end{array}\right] \equiv G_{\alpha_{1}-\frac{s b}{2}, \alpha_{3}-\frac{s^{\prime} b}{2}}\left[\begin{array}{cc}
\alpha_{3} & -\frac{b}{2} \\
\alpha_{4} & \alpha_{1}
\end{array}\right]
$$

These are very simple [42]

$$
\begin{array}{ll}
G_{++}=\frac{\sin \left(\pi b\left(\alpha_{4}+\alpha_{3}-\alpha_{1}-\frac{b}{2}\right)\right)}{\sin \left(\pi b\left(2 \alpha_{3}-b\right)\right)} & G_{+-}=\frac{\sin \left(\pi b\left(\alpha_{4}+\alpha_{3}+\alpha_{1}-\frac{3 b}{2}\right)\right)}{\sin \left(\pi b\left(2 \alpha_{3}-b\right)\right)} \\
G_{-+}=\frac{\sin \left(\pi b\left(\alpha_{3}+\alpha_{1}-\alpha_{4}-\frac{b}{2}\right)\right)}{\sin \left(\pi b\left(2 \alpha_{3}-b\right)\right)} & G_{--}=\frac{\sin \left(\pi b\left(\alpha_{3}-\alpha_{1}-\alpha_{4}+\frac{b}{2}\right)\right)}{\sin \left(\pi b\left(2 \alpha_{3}-b\right)\right)}
\end{array}
$$

which can also be written as

$$
G_{s_{1},-s_{2}}=s_{1} \frac{\sin \left(\pi b\left(\alpha_{4}+s_{1} \alpha_{3}+s_{2} \alpha_{1}-\left(1+s_{1}+s_{2}\right) \frac{b}{2}\right)\right)}{\sin \left(\pi b\left(2 \alpha_{3}-b\right)\right)} .
$$

Even more special cases involve a pair of degenerate fields. Creating them out of the identity is given by $\left(\alpha^{\prime}=\alpha-s b / 2\right)$

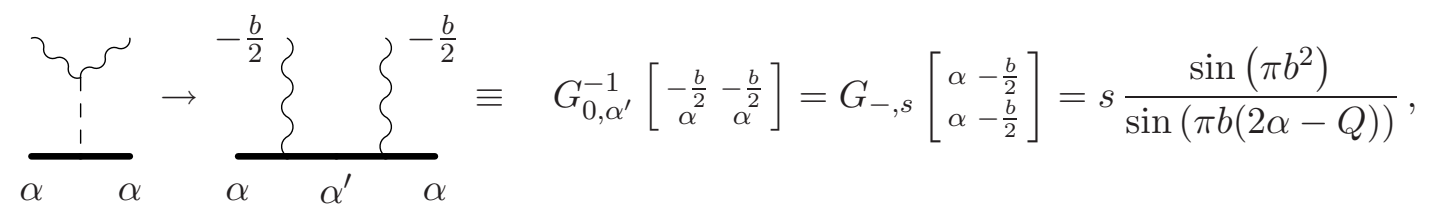

Analogously fusing them back to the identity

$$
\begin{aligned}
G_{\alpha^{\prime}, 0}\left[\begin{array}{cc}
-\frac{b}{2} & -\frac{b}{2} \\
\alpha^{2} & \alpha^{2}
\end{array}\right] & =G_{s,-}=s \frac{\sin \left(\pi b\left(2 \alpha^{\prime}-Q\right)\right)}{\sin \left(2 \pi b^{2}\right)}, \\
G_{\alpha, 0}\left[\begin{array}{cc}
-\frac{b}{2} & -\frac{b}{2} \\
\alpha^{\prime} & \alpha^{\prime}
\end{array}\right] & =G_{-s,-}=-s \frac{\sin (\pi b(2 \alpha-Q))}{\sin \left(2 \pi b^{2}\right)} .
\end{aligned}
$$

\section{B.1 Higher degenerate fields}

Other quantities we will need involve the higher degenerate fields with $-q b / 2$. The splitting of the identity into a pair of $-q b / 2$ fields can be done recursively

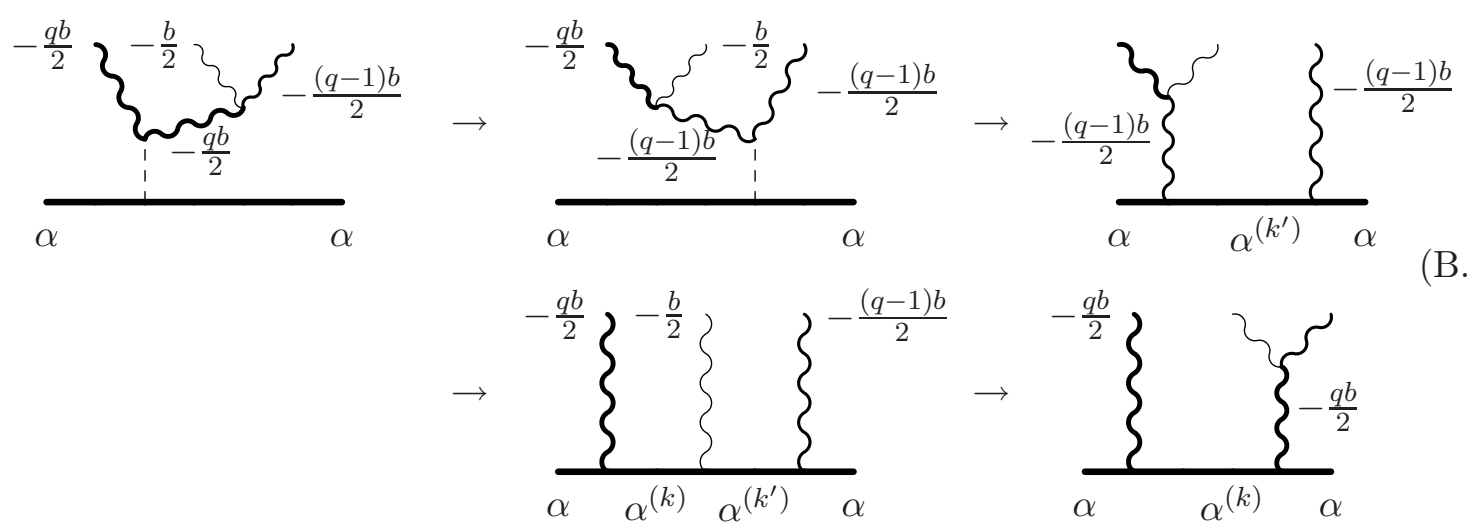


Using $\alpha^{(k)}=\alpha-k b / 2, \alpha^{\left(k^{\prime}\right)}=\alpha-m^{\prime} b / 2$ and $k=k^{\prime}+s_{q}$, we have for these fusion steps

$$
\begin{aligned}
& G_{0, \alpha-\frac{k b}{2}}\left[\begin{array}{c}
\alpha-\frac{q b}{2} \\
\alpha-\frac{q b}{2}
\end{array}\right]=G_{-\frac{q b}{2},-\frac{(q-1) b}{2}}\left[\begin{array}{cc}
-\frac{q b}{2} & -\frac{b}{2} \\
0 & -\frac{(q-1) b}{2}
\end{array}\right] G_{0, \alpha-\frac{k^{\prime} b}{2}}^{-1}\left[-\frac{(q-1) b}{2}-\frac{(q-1) b}{2}\right] \\
& \times G_{-\frac{(q-1) b}{2}, \alpha-\frac{k b}{2}}^{-1}\left[\begin{array}{cc}
-\frac{q b}{2} & -\frac{b}{2} \\
\alpha & \alpha-\frac{k^{\prime} b}{2}
\end{array}\right] G_{\alpha-\frac{k^{\prime} b}{2},-\frac{q b}{2}}\left[\begin{array}{cc}
-\frac{b}{2} & -\frac{(q-1) b}{2} \\
\alpha-\frac{k b}{2} & \alpha
\end{array}\right] \\
& =G_{+-}\left[\begin{array}{cc}
-\frac{q b}{2} & -\frac{b}{2} \\
0 & -\frac{(q-1) b}{2}
\end{array}\right] G_{0, \alpha-\frac{k^{\prime} b}{2}}\left[\begin{array}{c}
\alpha-\frac{(q-1) b}{2} \\
\alpha-\frac{(q-1) b}{2}
\end{array}\right] G_{-, s_{q}}\left[\begin{array}{cc}
\alpha-\frac{k^{\prime} b}{2} & -\frac{b}{2} \\
\alpha & -\frac{q b}{2}
\end{array}\right] G_{-s_{q},+}\left[\begin{array}{cc}
-\frac{(q-1) b}{2} & -\frac{b}{2} \\
\alpha & \alpha-\frac{k b}{2}
\end{array}\right]
\end{aligned}
$$

Using the explicit expressions for the fusion matrices we find

$$
G_{0, \alpha-\frac{k b}{2}}\left[\begin{array}{c}
\alpha-\frac{q b}{2} \\
\alpha-\frac{q b}{2}
\end{array}\right]=-s_{q} \frac{\sin ^{2}\left(\pi b^{2}\left(s_{q} q+k\right) / 2\right)}{\sin \left(\pi b^{2} q\right) \sin \left(\pi b\left(2 \alpha-\left(k^{\prime}+1\right) b\right)\right)} G_{0, \alpha-\frac{k^{\prime} b}{2}}\left[\begin{array}{c}
\alpha-\frac{(q-1) b}{2} \\
\alpha-\frac{(q-1) b}{2}
\end{array}\right] .
$$

Writing $k=s_{1}+\cdots+s_{q}$ we get for the full product

$$
G_{0, \alpha-\frac{k b}{2}}\left[\begin{array}{c}
\alpha-\frac{q b}{2} \\
\alpha-\frac{q b}{2}
\end{array}\right]=(-1)^{\frac{q+k}{2}} \prod_{i=1}^{q} \frac{\sin ^{2}\left(\pi b^{2}\left(i s_{i}+\left(s_{1}+\cdots+s_{i}\right)\right) / 2\right)}{\sin \left(\pi b^{2} i\right) \sin \left(\pi b\left(2 \alpha-\left(1+s_{1}+\cdots+s_{i-1}\right) b\right)\right)},
$$

which has to be summed over all orderings of the $s_{i}$.

For example, for $q=2$ summing over the choices of $s_{1}$ and $s_{2}$ gives

$$
\begin{aligned}
G_{0, \alpha-b}\left[\begin{array}{ll}
\alpha-b \\
\alpha-b
\end{array}\right] & =\frac{\sin \left(\pi b^{2}\right) \sin \left(2 \pi b^{2}\right)}{\sin (\pi b(2 \alpha-2 b)) \sin (\pi b(2 \alpha-b))} \\
G_{0, \alpha}\left[\begin{array}{l}
\alpha-b \\
\alpha-b
\end{array}\right] & =-\frac{\sin ^{3}\left(\pi b^{2}\right)}{\sin \left(2 \pi b^{2}\right) \sin (\pi b(2 \alpha-b))}\left(\frac{1}{\sin (\pi b(2 \alpha-2 b))}+\frac{1}{\sin (2 \pi b \alpha)}\right) \\
& =-\frac{\sin ^{2}\left(\pi b^{2}\right)}{\sin (\pi b(2 \alpha-2 b)) \sin (2 \pi b \alpha)} \\
G_{0, \alpha+b}\left[\begin{array}{l}
\alpha-b \\
\alpha-b
\end{array}\right] & =\frac{\sin \left(\pi b^{2}\right) \sin \left(2 \pi b^{2}\right)}{\sin (2 \pi b \alpha) \sin (\pi b(2 \alpha-b))}
\end{aligned}
$$

Similarly we can go in the opposite direction
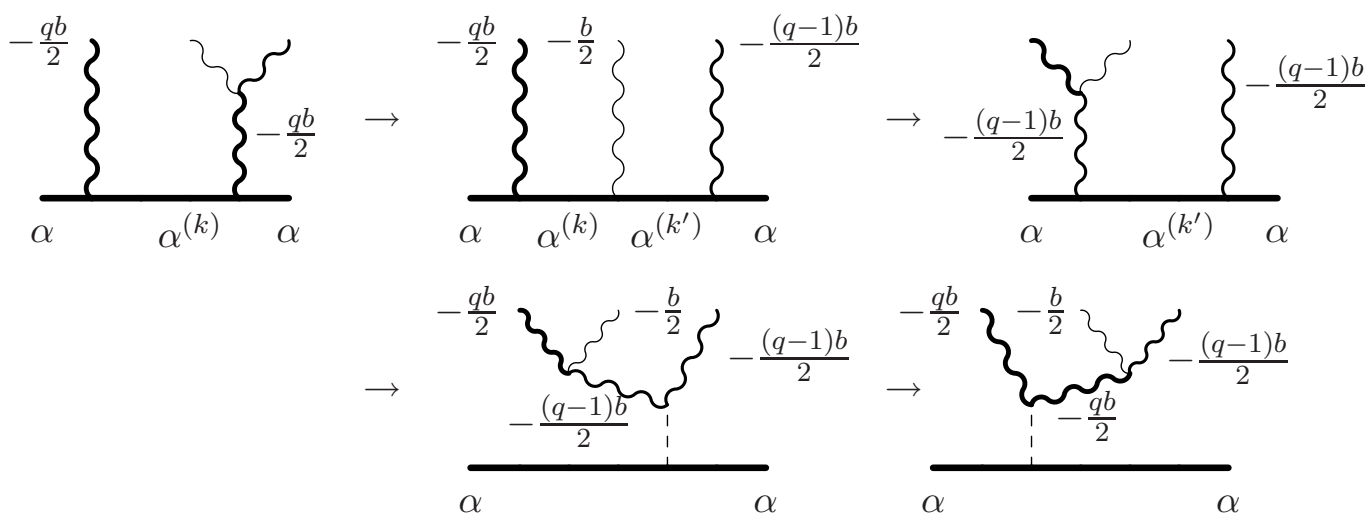

This is represented by the fusion matrices

$$
G_{\alpha-\frac{k b}{2}, 0}\left[\begin{array}{cc}
-\frac{q b}{2}-\frac{q b}{2} & \alpha
\end{array}\right]=G_{-\frac{q b}{2}, \alpha-\frac{k^{\prime} b}{2}}^{-1}\left[\begin{array}{cc}
-\frac{b}{2} & -\frac{(q-1) b}{2} \\
\alpha-\frac{k b}{2} & \alpha
\end{array}\right] G_{\alpha-\frac{k b}{2},-\frac{(q-1) b}{2}}\left[\begin{array}{cc}
-\frac{q b}{2} & -\frac{b}{2} \\
\alpha & \alpha-\frac{k^{\prime} b}{2}
\end{array}\right]
$$




$$
\begin{gathered}
\times G_{\alpha-\frac{k^{\prime} b}{2}, 0}\left[-\frac{(q-1) b}{2}-\frac{(q-1) b}{2}\right] G_{-\frac{(q-1) b}{2},-\frac{q b}{2}}\left[\begin{array}{cc}
-\frac{b}{2} & -\frac{(q-1) b}{2} \\
-\frac{q b}{2} & 0
\end{array}\right] \\
=G_{+,-s_{q}}\left[\begin{array}{cc}
\alpha-\frac{k b}{2} & -\frac{b}{2} \\
\alpha & -\frac{(q-1) b}{2}
\end{array}\right] G_{s_{q},-}\left[\begin{array}{cc}
-\frac{q b}{2} & -\frac{b}{2} \\
\alpha & \alpha-\frac{k^{\prime} b}{2}
\end{array}\right] G_{-+}\left[\begin{array}{cc}
-\frac{(q-1) b}{2} & -\frac{b}{2} \\
0 & -\frac{q b}{2}
\end{array}\right] G_{\alpha-\frac{k^{\prime} b}{2}, 0}\left[-\frac{(q-1) b}{\alpha}-\frac{(q-1) b}{2}\right]
\end{gathered}
$$

Explicitly this is

$$
G_{\alpha-\frac{k b}{2}, 0}\left[-\frac{q b}{2}-\frac{q b}{2}\right]=-s_{q} \frac{\sin ^{2}\left(\pi b\left(2 \alpha-\left(s_{q} q+k+2\right) \frac{b}{2}\right)\right)}{\sin \left(\pi b^{2}(q+1)\right) \sin (\pi b(2 \alpha-(k+1) b))} G_{\alpha-\frac{k^{\prime} b}{2}, 0}\left[-\frac{(q-1) b}{\alpha^{2}}-\frac{(q-1) b}{\alpha^{2}}\right] .
$$

Writing $k=s_{1}+\cdots+s_{q}$ we get for the full product

$$
(-1)^{\frac{q-k}{2}} \prod_{i=1}^{q} \frac{\sin ^{2}\left(\pi b\left(2 \alpha-\left(2+i s_{i}-\left(s_{1}+\cdots+s_{i}\right)\right) \frac{b}{2}\right)\right)}{\sin \left(\pi b^{2}(i+1)\right) \sin \left(\pi b\left(2 \alpha-\left(1+s_{1}+\cdots+s_{i}\right) b\right)\right)},
$$

which has to be summed over all orderings of the $s_{i}$.

For example, for $q=2$ summing over the choices of $s_{1}$ and $s_{2}$ gives

$$
\begin{aligned}
G_{\alpha-b, 0}\left[\begin{array}{cc}
-b & -b \\
\alpha & \alpha
\end{array}\right] & =\frac{\sin (\pi b(2 \alpha-2 b)) \sin (\pi b(2 \alpha-3 b))}{\sin \left(2 \pi b^{2}\right) \sin \left(3 \pi b^{2}\right)} \\
G_{\alpha, 0}\left[\begin{array}{cc}
-b & -b \\
\alpha & \alpha
\end{array}\right] & =-\frac{\sin (2 \pi b \alpha) \sin (\pi b(2 \alpha-2 b))[\sin (2 \pi b \alpha)+\sin (\pi b(2 \alpha-2 b))]}{\sin \left(2 \pi b^{2}\right) \sin \left(3 \pi b^{2}\right) \sin (\pi b(2 \alpha-b))} \\
& =-\frac{\sin (2 \pi b \alpha) \sin (\pi b(2 \alpha-2 b))}{\sin \left(\pi b^{2}\right) \sin \left(3 \pi b^{2}\right)} \\
G_{\alpha+b, 0}\left[\begin{array}{cc}
-b & -b \\
\alpha & \alpha
\end{array}\right] & =\frac{\sin (2 \pi b \alpha) \sin (\pi b(2 \alpha+b))}{\sin \left(2 \pi b^{2}\right) \sin \left(3 \pi b^{2}\right)}
\end{aligned}
$$

While we did not simplify these expressions in the general case, we did find by experimention the following useful formula for the product of two fusion steps with fixed $k$ (and checked it up to $q=5$ ).

$$
G_{0, \alpha-\frac{k b}{2}}^{-1}\left[\begin{array}{cc}
-\frac{q b}{2} & -\frac{q b}{2} \\
\alpha & \alpha^{2}
\end{array} G_{\alpha-\frac{k b}{2}, 0}\left[\begin{array}{cc}
-\frac{q b}{2} & -\frac{q b}{2} \\
\alpha^{2}
\end{array}\right]=\frac{-1}{\sum_{k^{\prime}} e^{\pi i k^{\prime} b Q}} \frac{\sin (\pi b(2 \alpha-k b-Q))}{\sin (\pi b(2 \alpha-Q))} .\right.
$$

The sum in the denominator is over $k^{\prime}=-q,-q+2, \ldots q$. Using this equation it is possible to derive the expression for the Wilson loop in the spin $j=q / 2$ representation (5.18) in section 5.2 .

Each of the pair of $-q b / 2$ degenerate fields can also be split recursively into $q$ of the basic degenerate fields
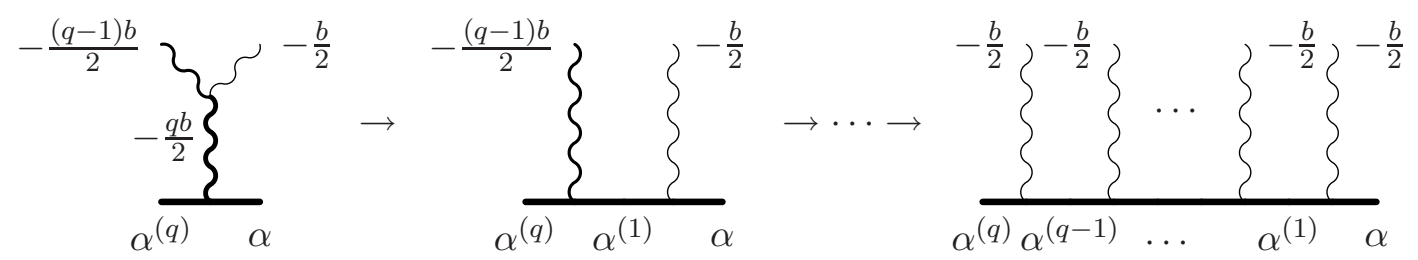
Taking $\alpha^{(q)}=\alpha-k b / 2$ with $k=\sum_{i=1}^{q} s_{i}$, we have for the first fusion step

$$
G_{-q b / 2, \alpha^{(1)}}^{-1}\left[\begin{array}{cc}
-\frac{(q-1) b}{2} & -\frac{b}{2} \\
\alpha^{(q)} & \alpha
\end{array}\right]=G_{+, s_{1}}\left[\begin{array}{cc}
\alpha & -\frac{b}{2} \\
\alpha^{(q)} & -\frac{(q-1) b}{2}
\end{array}\right]=\frac{\sin \left(\pi b^{2}(2 \alpha-b+(q s-k) b / 2)\right)}{\sin \left(\pi b^{2}(2 \alpha-b)\right)} .
$$

Splitting up the field with $-q b / 2$ into $q$ fields with $-b / 2$ is given by the product

$$
\frac{1}{\sin ^{q-1}\left(\pi b^{2}(2 \alpha-b)\right)} \prod_{i=2}^{q} \sin \left(\pi b^{2}\left(2 \alpha-b+\left(i s_{i}-\left(s_{1}+\cdots+s_{i}\right)\right) b / 2\right)\right) .
$$

Clearly for $s_{1}=\cdots=s_{q}$ the product is just unity.

We can also go in the other direction, fusing $q$ basic degenerate fields and projecting on the symmetric combination, which is a single $q b / 2$ field
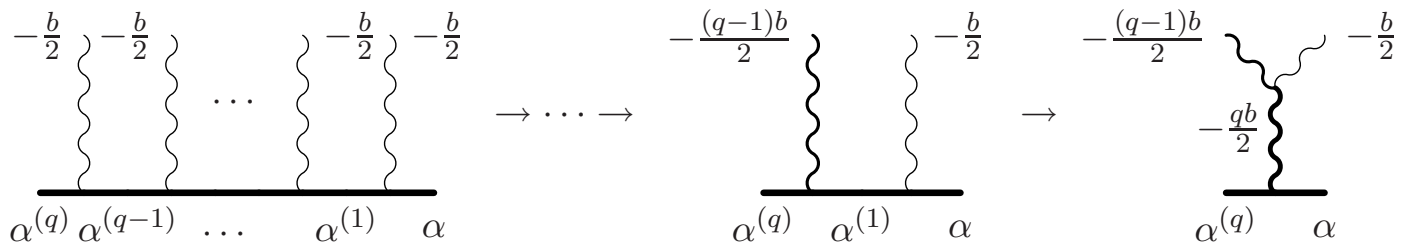

Taking $\alpha^{(q)}=\alpha-k b / 2$ with $k=\sum_{i=1}^{q} s_{i}$, we have for the last fusion step

$$
G_{\alpha^{(1)},-q b / 2}\left[\begin{array}{cc}
-\frac{(q-1) b}{2} & -\frac{b}{2} \\
\alpha^{(q)} & \alpha
\end{array}\right]=G_{s_{1}+}=\frac{\sin \left(\pi b^{2}\left(q+s_{1} k\right) / 2\right)}{\sin \left(\pi b^{2} q\right)} .
$$

Doing this $q$ times we get the product

$$
\prod_{i=2}^{q} \frac{\sin \left(\pi b^{2}\left(i+s_{i}\left(s_{1}+\cdots+s_{i}\right)\right) / 2\right)}{\sin \left(\pi b^{2} i\right)} .
$$

Though this is not manifest, the product on the right hand side is symmetric under exchange of any of the $s_{i} \leftrightarrow s_{j}$. If we take $s_{1}, \ldots, s_{(q+k) / 2}$ to be positive and the rest to be negative, then the numerator of the first $(q+k) / 2$ terms will be $\sin \left(\pi b^{2} i\right)$ and the subsequent ones $\sin \left(\pi b^{2}(2 i-q-k) / 2\right)$. The first $(q+k) / 2$ will cancel against the denominator giving

$$
\prod_{j=1}^{\frac{q-|k|}{2}} \frac{\sin \left(\pi b^{2} j\right)}{\sin \left(\pi b^{2}\left(j+\frac{q+|k|}{2}\right)\right)} .
$$

Clearly for $s_{1}=\cdots=s_{q}$ the product is just unity.

For example for $q=2$

$$
G_{\alpha^{(1)},-b}\left[\begin{array}{cc}
-\frac{b}{2} & -\frac{b}{2} \\
\alpha-\frac{\left(s_{1}+s_{2}\right) b}{2} & \alpha
\end{array}\right]=\frac{\sin \left(\pi b^{2}\left(3+s_{1} s_{2}\right) / 2\right)}{\sin \left(2 \pi b^{2}\right)}= \begin{cases}1, & s_{1}=s_{2} \\
\frac{1}{2 \cos \left(\pi b^{2}\right)}, & s_{1}=-s_{2} .\end{cases}
$$

Combining three $-b / 2$ fields to a single $-3 b / 2$ field gives

$$
\frac{\sin \left(\pi b^{2}\left(3+s_{1} s_{2}\right) / 2\right) \sin \left(\pi b^{2}\left(4+s_{3}\left(s_{1}+s_{2}\right)\right) / 2\right)}{\sin \left(2 \pi b^{2}\right) \sin \left(3 \pi b^{2}\right)}= \begin{cases}1, & s_{1}=s_{2}=s_{3} \\ \frac{\sin \left(\pi b^{2}\right)}{\sin \left(3 \pi b^{2}\right)}, & \text { otherwise. }\end{cases}
$$


Open Access. This article is distributed under the terms of the Creative Commons Attribution Noncommercial License which permits any noncommercial use, distribution, and reproduction in any medium, provided the original author(s) and source are credited.

\section{References}

[1] C. Montonen and D.I. Olive, Magnetic monopoles as gauge particles?, Phys. Lett. B 72 (1977) 117 [SPIRES].

[2] E. Witten and D.I. Olive, Supersymmetry algebras that include topological charges, Phys. Lett. B 78 (1978) 97 [SPIRES].

[3] H. Osborn, Topological charges for $\mathcal{N}=4$ supersymmetric gauge theories and monopoles of spin 1, Phys. Lett. B 83 (1979) 321 [SPIRES].

[4] P. Goddard, J. Nuyts and D.I. Olive, Gauge theories and magnetic charge, Nucl. Phys. B 125 (1977) 1 [SPIRES].

[5] N. Seiberg and E. Witten, Monopoles, duality and chiral symmetry breaking in $\mathcal{N}=2$ supersymmetric QCD, Nucl. Phys. B 431 (1994) 484 [hep-th/9408099] [SPIRES].

[6] P.C. Argyres, $S$-duality and global symmetries in $N=2$ supersymmetric field theory, Adv. Theor. Math. Phys. 2 (1998) 293 [hep-th/9706095] [SPIRES].

[7] E. Witten, Solutions of four-dimensional field theories via M-theory, Nucl. Phys. B 500 (1997) 3 [hep-th/9703166] [SPIRES].

[8] D. Gaiotto, $\mathcal{N}=2$ dualities, arXiv:0904.2715 [SPIRES].

[9] D. Gaiotto, G.W. Moore and A. Neitzke, Wall-crossing, Hitchin systems and the WKB approximation, arXiv:0907.3987 [SPIRES].

[10] V. Pestun, Localization of gauge theory on a four-sphere and supersymmetric Wilson loops, arXiv:0712.2824 [SPIRES].

[11] N.A. Nekrasov, Seiberg-Witten prepotential from instanton counting, Adv. Theor. Math. Phys. 7 (2004) 831 [hep-th/0206161] [SPIRES].

[12] N. Nekrasov and A. Okounkov, Seiberg-Witten theory and random partitions, hep-th/0306238 [SPIRES].

[13] L.F. Alday, D. Gaiotto and Y. Tachikawa, Liouville correlation functions from four-dimensional gauge theories, arXiv:0906.3219 [SPIRES].

[14] J. Teschner, Liouville theory revisited, Class. Quant. Grav. 18 (2001) R153 [hep-th/0104158] [SPIRES].

[15] J. Teschner, A lecture on the Liouville vertex operators, Int. J. Mod. Phys. A 19S2 (2004) 436 [hep-th/0303150] [SPIRES].

[16] J. Teschner, Nonrational conformal field theory, arXiv:0803.0919 [SPIRES].

[17] J.M. Maldacena, Wilson loops in large-N field theories, Phys. Rev. Lett. 80 (1998) 4859 [hep-th/9803002] [SPIRES].

[18] S.-J. Rey and J.-T. Yee, Macroscopic strings as heavy quarks in large- $N$ gauge theory and anti-de Sitter supergravity, Eur. Phys. J. C 22 (2001) 379 [hep-th/9803001] [SPIRES]. 
[19] J.K. Erickson, G.W. Semenoff and K. Zarembo, Wilson loops in $\mathcal{N}=4$ supersymmetric Yang-Mills theory, Nucl. Phys. B 582 (2000) 155 [hep-th/0003055] [SPIRES].

[20] N. Drukker and D.J. Gross, An exact prediction of $\mathcal{N}=4$ SUSYM theory for string theory, J. Math. Phys. 42 (2001) 2896 [hep-th/0010274] [SPIRES].

[21] A. Kapustin, Wilson-'t Hooft operators in four-dimensional gauge theories and S-duality, Phys. Rev. D 74 (2006) 025005 [hep-th/0501015] [SPIRES].

[22] A. Kapustin and E. Witten, Electric-magnetic duality and the geometric Langlands program, hep-th/0604151 [SPIRES].

[23] J. Gomis, T. Okuda and D. Trancanelli, Quantum 't Hooft operators and S-duality in $\mathcal{N}=4$ super Yang-Mills, arXiv:0904.4486 [SPIRES].

[24] J. Gomis and T. Okuda, S-duality, 't Hooft operators and the operator product expansion, JHEP 09 (2009) 072 [arXiv:0906.3011] [SPIRES].

[25] N. Drukker, D.R. Morrison and T. Okuda, Loop operators and S-duality from curves on Riemann surfaces, JHEP 09 (2009) 031 [arXiv:0907.2593] [SPIRES].

[26] A. Kapustin, Holomorphic reduction of $\mathcal{N}=2$ gauge theories, Wilson-'t Hooft operators and S-duality, hep-th/0612119 [SPIRES].

[27] J. Teschner, On the relation between quantum Liouville theory and the quantized Teichmueller spaces, Int. J. Mod. Phys. A 19S2 (2004) 459 [hep-th/0303149] [SPIRES].

[28] J. Teschner, From Liouville theory to the quantum geometry of Riemann surfaces, in Prospects in mathematical physics, Contemp. Math. 437 (2007) 231, American Mathematical Society, Providence U.S.A. (2007) [hep-th/0308031] [SPIRES].

[29] J. Teschner, An analog of a modular functor from quantized Teichmüller theory, in Handbook of Teichmüller theory. Volume I, IRMA Lect. Math. Theor. Phys. 11 (2007) 685, European Mathematical Society, Zürich Switzerland (2007) [math.QA/0510174 [SPIRES].

[30] H.L. Verlinde, Conformal field theory, $2 D$ quantum gravity and quantization of Teichmüller space, Nucl. Phys. B 337 (1990) 652 [SPIRES].

[31] L.F. Alday, D. Gaiotto, S. Gukov, Y. Tachikawa and H. Verlinde, Loop and surface operators in $\mathcal{N}=2$ gauge theory and Liouville modular geometry, arXiv:0909.0945 [SPIRES].

[32] A.A. Belavin, A.M. Polyakov and A.B. Zamolodchikov, Infinite conformal symmetry in two-dimensional quantum field theory, Nucl. Phys. B 241 (1984) 333 [SPIRES].

[33] H. Dorn and H.J. Otto, Two and three point functions in Liouville theory, Nucl. Phys. B 429 (1994) 375 [hep-th/9403141] [SPIRES].

[34] A.B. Zamolodchikov and A.B. Zamolodchikov, Structure constants and conformal bootstrap in Liouville field theory, Nucl. Phys. B 477 (1996) 577 [hep-th/9506136] [SPIRES].

[35] D. Gaiotto, Asymptotically free $\mathcal{N}=2$ theories and irregular conformal blocks, arXiv: 0908.0307 [SPIRES].

[36] M. Dehn, On curve systems on two-sided surfaces, with application to the mapping problem, in Papers on group theory and topology, translated from the German by J. Stillwell, Springer-Verlag, New York U.S.A. (1987), pg. 234.

[37] W.P. Thurston, On the geometry and dynamics of diffeomorphisms of surfaces, Bull. Am. Math. Soc. 19 (1988) 417. 
[38] A. Bytsko and J. Teschner, The integrable structure of nonrational conformal field theory, arXiv:0902. 4825 [SPIRES].

[39] E.P. Verlinde, Fusion rules and modular transformations in $2 D$ conformal field theory, Nucl. Phys. B 300 (1988) 360 [SPIRES].

[40] G.W. Moore and N. Seiberg, Classical and quantum conformal field theory, Commun. Math. Phys. 123 (1989) 177 [SPIRES].

[41] G.W. Moore and N. Seiberg, Lectures on RCFT, presented at Trieste Spring School, Trieste Italy (1989) [SPIRES].

[42] B. Ponsot and J. Teschner, Liouville bootstrap via harmonic analysis on a noncompact quantum group, hep-th/9911110 [SPIRES].

[43] R.C. Penner, The action of the mapping class group on curves in surfaces, Enseign. Math. 30 (1984) 39.

[44] P.G. Zograf and L.A. Takhtadzhyan, On the Liouville equation, accessory parameters and the geometry of Teichmüller space for Riemann surfaces of genus 0, Mat. Sbornik 132(174) (1987) 147 [Math. USSR Sb. 60(1) (1988) 143].

[45] P.G. Zograf and L.A. Takhtadzhyan, On the uniformization of Riemann surfaces and on the Weil-Petersson metric on the Teichmüller and Schottky spaces, Mat. Sbornik 132(174) (1987) 304 [Math. USSR Sb. 60(2) (1988) 297].

[46] R.C. Penner, The decorated Teichmüller space of punctured surfaces, Commun. Math. Phys. 113 (1987) 299.

[47] V.V. Fock, Dual Teichmüller spaces, dg-ga/9702018.

[48] R.C. Penner, Weil-Petersson volumes, J. Diff. Geom. 35 (1992) 559.

[49] L.A. Takhtajan, Uniformization, local index theorem, and geometry of the moduli spaces of Riemann surfaces and vector bundles, in Theta functions - Bowdoin 1987, Proc. Symp. Pure Math. 49, U.S.A. (1989).

[50] L. Takhtajan and P. Zograf, Hyperbolic 2-spheres with conical singularities, accessory parameters and Kähler metrics on $\mathcal{M}_{0, n}$, Trans. Am. Math. Soc. 355 (2003) 1857 [math.CV/0112170] [SPIRES].

[51] L. Chekhov and V.V. Fock, A quantum Teichmüller space, Theor. Math. Phys. 120 (1999) 1245 [Teor. Mat. Fiz. 120 (1999) 511] [math.QA/9908165] [SPIRES].

[52] R.M. Kashaev, Quantization of Teichmüller spaces and the quantum dilogarithm, Lett. Math. Phys. 43 (1998) 105 [SPIRES].

[53] V.V. Fock and A.B. Goncharov, The quantum dilogarithm and representations of quantum cluster varieties, Invent. Math. 175 (2009) 223.

[54] L.O. Chekhov and V.V. Fock, Observables in $3 D$ gravity and geodesic algebras, Czech. J. Phys. 50 (2000) 1201 [SPIRES].

[55] G. 't Hooft, On the phase transition towards permanent quark confinement, Nucl. Phys. B 138 (1978) 1 [SPIRES].

[56] A. Kapustin and N. Saulina, The algebra of Wilson-'t Hooft operators, Nucl. Phys. B 814 (2009) 327 [arXiv:0710.2097] [SPIRES]. 
[57] L. Chekhov, Lecture notes on quantum Teichmüller theory, arXiv:0710.2051.

[58] L.O. Chekhov, Orbifold Riemann surfaces and geodesic algebras, J. Phys. A 42 (2009) 304007.

[59] N. Drukker, S. Giombi, R. Ricci and D. Trancanelli, More supersymmetric Wilson loops, Phys. Rev. D 76 (2007) 107703 [arXiv:0704 .2237] [SPIRES].

[60] N. Drukker, S. Giombi, R. Ricci and D. Trancanelli, Supersymmetric Wilson loops on $S^{3}$, JHEP 05 (2008) 017 [arXiv:0711.3226] [SPIRES].

[61] N. Wyllard, $A_{N-1}$ conformal Toda field theory correlation functions from conformal $\mathcal{N}=2$ $S U(N)$ quiver gauge theories, JHEP 11 (2009) 002 [arXiv:0907.2189] [SPIRES].

[62] V. Fock and A. Goncharov, Moduli spaces of local systems and higher Teichmüller theory, Inst. Hautes Études Sci. Publ. Math. 103 (2006) 1.

[63] N.J. Hitchin, Lie groups and Teichmüller space, Topology 31 (1992) 449.

[64] T.J. Hollowood, A. Iqbal and C. Vafa, Matrix models, geometric engineering and elliptic genera, JHEP 03 (2008) 069 [hep-th/0310272] [SPIRES].

[65] A. Iqbal, C. Kozcaz and C. Vafa, The refined topological vertex, JHEP 10 (2009) 069 [hep-th/0701156] [SPIRES].

[66] E.W. Barnes, The theory of the double gamma function, Proc. Roy. Soc. Lond. 66 (1899) 265.

[67] T. Shintani, On a Kronecker limit formula for real quadratic fields, J. Fac. Sci. Tokyo (Sect. 1A Math.) 24 (1977) 167. 